COMPREHENSIVE INVITED REVIEW

\title{
MAMMALIAN SULFUR AMINO ACID METABOLISM: A NEXUS BETWEEN REDOX REGULATION, NUTRITION, EPIGENETICS AND \\ DETOXIFICATION
}

María A. Pajares ${ }^{1,2, *}$ and Dolores Pérez-Sala ${ }^{1}$

${ }^{1}$ Department of Chemical and Physical Biology, Centro de Investigaciones Biológicas (CSIC), Ramiro de Maeztu 9, 28040 Madrid, Spain.

${ }^{2}$ Molecular Hepatology Group, Instituto de Investigación Sanitaria La Paz (IdiPAZ), Paseo de la Castellana 261, 28046 Madrid, Spain.

*To whom correspondence should be addressed at: Dept. of Chemical and Physical Biology, Centro de Investigaciones Biológicas (CSIC), Ramiro de Maeztu 9, 28040 Madrid, Spain. Phone: +34-918373112. Email: mapajares@cib.csic.es or ma.pajares@csic.es

Running head: Interplays of sulfur amino acid metabolism

Keywords: detoxification mechanisms, epigenetic methylations, glutathione, one carbon metabolism, post-translational modifications, transsulfuration.

Word count: 17132 (excluding references and figure legends)

Reference number: 426

Greyscale illustrations: 12

Color illustrations: 5 (online 5 and hardcopy 0 )

Tables: 1

Reviewing Editors: Sandrine Boschi-Muller, Marilene Demasi, Milos Filipovich, Gabriel Gojon, Ignazio Grattagliano, Bindu Paul, Peter Rose, Juan Sastre and Daniel Vaiman. 
Pajares and Pérez-Sala

\section{ABSTRACT}

Significance. Transsulfuration allows conversion of methionine into cysteine using homocysteine as an intermediate. This pathway produces S-adenosylmethionine, a key metabolite for cell function, and provides $50 \%$ of the cysteine needed for hepatic glutathione synthesis. The route requires the intake of essential nutrients (e.g. methionine, vitamins) and is regulated by their availability. Transsulfuration presents multiple interconnections with epigenetics, ATP and glutathione synthesis, polyol and pentose phosphate pathways and detoxification that rely mostly in the exchange of substrates or products. Major hepatic diseases, rare diseases and sensorineural disorders, among others that concur with oxidative stress, present impaired transsulfuration. Recent advances. In contrast to the classical view, a nuclear branch of the pathway, potentiated under oxidative stress, is emerging. Several transsulfuration proteins regulate gene expression, suggesting moonlighting activities. Additionally, abnormalities in homocysteine metabolism link nutrition and hearing loss. Critical issues. Knowledge about the cross-regulation between pathways is mostly limited to the hepatic availability/removal of substrates and inhibitors. However, advances regarding protein-protein interactions involving oncogenes, identification of several posttranslational modifications and putative moonlighting activities expand the potential impact of transsulfuration beyond methylations and homocysteine. Future directions. Increasing the knowledge on transsulfuration outside the liver, understanding the protein-protein interaction networks involving these enzymes, the functional role of their post-translational modifications or the mechanisms controlling their nucleocytoplasmic shuttling may provide further insights into the pathophysiological implications of this pathway, allowing design of new therapeutic interventions. 


\section{INTRODUCTION}

2. OVERVIEW OF THE MAMMALIAN TRANSSULFURATION PATHWAY

2.1. S-adenosylmethionine synthesis

2.2. Selected examples of genes and enzymes of transmethylation

2.3. Homocysteine synthesis and catabolism

\section{REGULATION OF TRANSSULFURATION}

3.1. Regulation of transsulfuration by the diet

3.2. Regulation by metabolites of transsulfuration and related pathways

3.3. New players identified through protein-protein interactions

3.4. Regulatory mechanisms through post-translational modifications

4. EPIGENETIC MODIFICATIONS DEPENDENT ON TRANSSULFURATION

4.1. Expression of epigenetic methyltransferases

4.2. Main features of epigenetic methyltransferases

\section{DEREGULATION OF TRANSSULFURATION IN PATHOLOGY}

5.1. Cancer and hepatocellular carcinoma

5.2. Ethanol abuse

5.3 Acute liver injury

5.4. Wilson disease

5.5. Hearing loss

6. DETOXIFICATION MECHANISMS LINKED TO TRANSSULFURATION

6.1. Aldo-ketoreductases

6.2. Glutathione S-transferases

6.3. Thiopurine methyltransferase

\section{HUMAN MUTATIONS IN TRANSSULFURATION}

7.1. Human mutations identified in transsulfuration genes

7.2. Impact of mutations in association and subcellular localization

8. INTEGRATED VIEW OF TRANSSULFURATION

9. CONCLUSIONS AND FUTURE DIRECTIONS 
Pajares and Pérez-Sala

\section{INTRODUCTION}

In the mid- $20^{\text {th }}$ century intermediary metabolism was a subject of intense research. Elucidation of most steps in these routes and their essential regulatory mechanisms lead us to think that nothing much was left to discover, hence becoming a "classic" topic. At that time the dependence of tumor cells on glutamine and methionine was described, and initial attempts to understand this phenomenon were made $(88,244,352)$. The existence of global DNA hypomethylation in tumor cells was later reported and shown to involve a decrease in the levels of a methionine derivative, Sadenosylmethionine (AdoMet, classically named SAM), the main cellular methyl donor $(78,193)$. Afterwards, the concomitant existence of hypermethylation at specific DNA segments, frequently corresponding to promoters of tumor suppressors, was demonstrated (285) and additional connections between metabolism and cancer identified (284). Lately, research carried out in fields such as autophagy, inflammation or cardiovascular disease have contributed also to recall on the importance of sulfur amino acid metabolism renewing the interest in this field.

Identification of hyperhomocysteinemia (HHcy) as an independent risk factor for cardiovascular disease spurred research towards homocysteine (Hcy) metabolism (372). However, the number of pathologies associated with impaired sulfur amino acid metabolism and that concur with oxidative stress extends far beyond including, among others, several types of cancer, hearing loss, psoriasis, cirrhosis, Wilson or Alzheimer's diseases $(72,120,141,175,242,316)$. As will be described, this is not only due to the large number of AdoMet-dependent processes, but also due to connections of transsulfuration within one carbon metabolism and with epigenetics, detoxification and overall nutrition. As for other important metabolic pathways, most of the accumulated knowledge in this field was obtained from studies using the liver and hepatocytes. 
Pajares and Pérez-Sala

Hence, the role of sulfur amino acid metabolism in other tissues or cells and their associated pathologies has been probably overlooked or just assumed to be similar in every context. Nevertheless, analyses of gene expression patterns in human and rodent tissues demonstrate the existence of the pathway in most organs/cells, the differences relying mainly in the methionine adenosyltransferase (MAT) and methyltransferase genes expressed.

Mammalian sulfur amino acid metabolism is centered around the methionine cycle and the pathway allowing conversion of Hcy into cysteine (Fig. 1). This last route is usually named transsulfuration in mammals, but reverse transsulfuration in other organisms where the term transsulfuration is reserved for the opposite reactions converting cysteine to Hcy (393). In some instances, transsulfuration is considered to comprise all the steps that allow the synthesis of cysteine from methionine and, for simplicity, we will follow this rule. The pathway is fed by methionine, the amino acid discovered in 1923 by John H. Mueller, and whose ingestion proved to be essential for adequate rat development (395). In contrast, cysteine, discovered in 1884 (18), becomes semi-essential due to transsulfuration. For this purpose, the methylene and the methyl groups bound to the sulfur atom of the methionine lateral chain need to be removed. Steps towards this end involve the synthesis and hydrolysis of several metabolites, AdoMet, S-adenosylhomocysteine (AdoHcy), Hcy and cystathionine. Among them, AdoMet synthesis is essential for life, and hence the MAT gene appears in the genome of the first synthetic organism (147).

Hcy, the third mammalian sulfur amino acid, is known since 1932 as a byproduct of the incubation of methionine with sulfuric acid (32), and emerges here as a transsulfuration intermediate. Methionine and cysteine have a proteinogenic role and can be targets for modifications $(25,386)$. In contrast, Hcy is a non-proteinogenic amino 
Pajares and Pérez-Sala

acid that can modify proteins post-translationally (152). Therefore, its concentration must be controlled to preclude not only lysine N-homocysteinylation and protein aggregation, but also activation by phosphorylation of protein kinase RNA-like endoplasmic reticulum (ER) kinase and ER stress and, in turn, phosphorylation and nuclear accumulation of Nrf2, a major redox regulator of gene expression (310). This control is achieved by Hcy export to the plasma and its use for remethylation, cystathionine and cysteine synthesis.

As will be discussed, several amino acids and metabolites of transsulfuration are key for epigenetic modifications, glutathione synthesis and detoxification reactions. Importantly, the synthesis and levels of these metabolites are intrinsically linked to nutritional intakes and the cellular redox status. Additionally, redox-sensitive transcription factors (i.e. NFKB, AP-1) and stress kinases (i.e. JNK) directly or indirectly regulate transsulfuration during redox stress, together with the changes in redox pairs (i.e. GSH/GSSG, NADH/NAD ${ }^{+}$). Detailed knowledge of the crossregulation linking all these processes is therefore needed to understand the impact that impaired transsulfuration exerts on many essential aspects of Cell Biology. 
Pajares and Pérez-Sala

\section{OVERVIEW OF THE MAMMALIAN TRANSSULFURATION PATHWAY}

Elucidation of the reactions that allow conversion of methionine into cysteine involve Cantoni's discoveries of MAT and AdoMet $(39,40)$. There are three mammalian MATs that catalyze AdoMet synthesis from methionine and ATP, which is the first and rate-limiting step of transsulfuration (Fig. 1). Identification of AdoMet and deciphering its structure proved to be essential in Biology, where dozens of processes depend on this metabolite as donor of a variety of groups $(41,194,248,351)$. Therefore, AdoMet becomes the first branch point in transsulfuration.

After decarboxylation, AdoMet serves as the aminopropyl donor for the synthesis of the polyamines spermidine and spermine (248). This process also generates 5'-methylthioadenosine (MTA), which inhibits a number of methyltransferases and is recycled back into methionine through the methionine salvage pathway (6). Furthermore, AdoMet is subjected to reductive cleavage through Fe-S centers to provide the 5'-deoxyadenosyl group needed by the eight human SAM radical proteins, as reviewed elsewhere (194) (Fig. 1). These reactions also produce methionine, which can reenter transsulfuration. Quantitatively, polyamine synthesis and SAM radical proteins consume $<10 \%$ of hepatic AdoMet, whereas most of the 6-8 g of AdoMet synthesized daily in humans are used in methylation reactions $(41,213,258,260)$. Nitrogen, sulfur, carbon or oxygen atoms of a large variety of substrates are the recipients of the methyl group donated by AdoMet in transmethylation reactions (41), which constitute the second step of transsulfuration (Fig. 1). Transmethylations produce AdoHcy, the demethylated form of AdoMet, which is a potent inhibitor of many methyltransferases (57). Hence, the importance of the AdoMet/AdoHcy ratio, also named the "methylation index". Adequate values of this index are maintained by AdoHcy elimination, which is the role of the next step catalyzed by AdoHcy hydrolase 
Pajares and Pérez-Sala

or adenosylhomocysteinase (AHCY) (163). However, the reversibility of this reaction emphasizes the need to eliminate the Hcy and adenosine produced to preclude accumulation of the transmethylation inhibitor AdoHcy.

Transsulfuration reaches then a double branch point, represented by adenosine and Hcy (Fig. 1). Adenosine is used by adenosine deaminase (ADA) or adenosine kinase (ADK), leading to inosine and AMP, respectively. Conversely, Hcy is recycled back into methionine by remethylation, a process for which three methyl donors can be utilized, and that closes the methionine cycle. Hcy remethylation by methionine synthase (MTR) depends on methylcobalamin (vitamin $\mathrm{B}_{12}$ ) and requires 5methyltetrahydrofolate (MTHF) as methyl donor, thus linking transsulfuration with the folate cycle (361). The other two possibilities for Hcy remethylation involve highly similar homotetrameric enzymes, betaine homocysteine S-methyltransferase (BHMT) and BHMT2, that obtain the methyl group from betaine and S-methylmethionine, respectively $(90,356)$. The source of these methyl donors is the diet $(66,185)$, although mitochondrial choline oxidation also produces betaine (318). Hcy catabolism through transsulfuration involves its conjugation with serine or cysteine to synthesize cystathionine in a reaction catalyzed by cystathionine $\beta$-synthase (CBS) $(89,360)$. Hydrolysis of cystathionine into cysteine constitutes the last reaction of transsulfuration that is driven by cystathione $\gamma$-lyase or cystathionase (CTH) $(347,353)$. Both CBS and $\mathrm{CTH}$ require pyridoxal phosphate (vitamin $\left.\mathrm{B}_{6}\right)$ as cofactor $(30,121)$ and, when using cysteine as substrate, synthesize hydrogen sulfide $\left(\mathrm{H}_{2} \mathrm{~S}\right)$, an important mediator in cellular signaling and cytoprotection $(15,393)$. Thus, both enzymes provide additional connections with nutrition, redox regulation and signaling. Nonetheless, a reduced Hcy carrier can export the Hcy excess to the plasma (27), if required. 
Pajares and Pérez-Sala

Intracellular levels of cysteine may reach $90-100 \mu \mathrm{M}$ as a consequence of both dietary intake and transsulfuration (382). Cysteine is then used in several processes, including protein, glutathione and taurine synthesis. Hepatic synthesis of glutathione, the main low molecular weight antioxidant and free radical scavenger, utilizes $\sim 50 \%$ of the cysteine produced through transsulfuration $(256,382)$. The process involves two ATP consuming steps (247). In the rate-limiting reaction, cysteine is bound to the $\gamma$ carboxyl group of glutamic acid by heterodimeric $\gamma$-glutamylcysteine synthetase or glutamate cysteine ligase (GCL). Later on, glycine is added to $\gamma$-glutamylcysteine by homodimeric glutathione synthetase (GSS), rendering the reduced form of glutathione (GSH). GSH feedback regulates its own synthesis by inhibiting GCL, whereas expression of GCL subunits is regulated by Nrf2 $(178,310)$.

Intracellular GSH levels are in the millimolar range, reaching 5-10 $\mathrm{mM}$ in the liver $(5,424)$. During detoxification and free radical scavenging GSH is conjugated and oxidized into GSSG, respectively. GSSG is reduced back into GSH by glutathione reductase (GSR) using NADPH. According to different methodologies, the GSH/GSSG ratio is normally kept between $50-300(5,71,424)$, exerting a preeminent role in the redox control of proteins, including some transsulfuration enzymes.

\subsection{S-adenosylmethionine synthesis}

According to protein function, the first group of genes in transsulfuration includes the three genes involved in AdoMet synthesis, named MAT1A, MAT2A and $\operatorname{MAT2B}$ (Fig. 2). The highly similar MAT $\alpha 1$ and MATa2 catalytic subunits $(84 \%$ identity) are encoded by $M A T 1 A$ and $M A T 2 A$, respectively $(125,183,277)$, whereas $M A T 2 B$ codifies for the unrelated regulatory MAT $\beta$ subunit (204). Basic information concerning these genes, including their chromosomal localization or the presence of SNPs is shown in Table 1. In hepatoma cells, several MAT2B splicing forms (V1, V2, 
Pajares and Pérez-Sala

V2a and V2b) have been reported, which differ mainly in their N-terminal, although V2a and V2b also present internal deletions of the sequence $(398,404)$. Recently, two alternative splicing forms have been reported for $M A T 2 A$ that differ in intron 8 retention/elimination (287).

Expression of MAT genes differs among tissues and during development, $M A T 2 A$ being preferentially expressed in extrahepatic tissues, fetal liver and tumor cells (111,366). MAT2B is expressed in most tissues following a similar pattern $(232,404)$. In contrast, expression of MAT1A is maximal in human and rodent adult liver, whereas intermediate levels are detected in pancreas and lung and low levels occur in other rodent tissues $(216,311,366)$. In normal liver, this expression pattern correlates with MAT1A promoter hypomethylation and hyperacetylation of its associated histones, whereas MAT2A promoter hypermethylation and histone hypoacetylation is observed $(366,367)$. Conversely, in extrahepatic tissues MAT1A and MAT2A promoters present the opposite pattern of modification, but no such information is available for $M A T 2 B$.

Several studies have analyzed the promoters of the MAT genes (Fig. 2). Human and rodent MAT1A promoters include a variety of cis-acting elements whose functionality has been partially determined and to which redox-sensitive transcription factors bind (Fig. 2A). Among them, the role of glucocorticoid responsive elements was inferred from the induction of hepatic Matla mRNA by triamcinolone and dexamethasone (112). Positive regulation of the rat promoter activity was exerted by a member of the NF-1 protein family, whereas negative regulation was ascribed to differential binding of HNF factors in liver and hepatoma (10). In the human MATIA promoter, upregulation by triiodothyronine was shown to involve binding of thyroid receptor TR $\beta$ to $\mathrm{C} / \mathrm{EBP}$ sites (396). $\mathrm{C} / \mathrm{EBP} \beta$ binding and promoter hypomethylation were also responsible for increased expression in the mouse from fetal day 14 onward 
Pajares and Pérez-Sala

(148), as expected from the crucial role of these factors in liver development and regeneration. The MAT1A promoter also contains an E-box to which MAT $\alpha 1 / \mathrm{Mnt}$ bind in normal liver. However, a switch towards cMyc/MafG/cMaf binding for MAT1A repression occurs upon induction of cholestasis and the associated oxidative stress (408). MAT $\alpha 1$ and prohibitin 1 (PHB1), the mitochondrial chaperone that folds respiratory chain proteins, positively regulate each other expression in normal liver. Nevertheless, the change in occupancy of their E-boxes as described above downregulates both genes (96).

Human MAT2A promoter presents a variety of elements for binding of several factors that are redox-sensitive (Fig. 2B). Enhanced MAT2A expression in hepatocellular carcinoma (HCC) and HepG2 cells occurs upon binding of Sp1, cMyb, NFkB and AP-1, $(406,409)$, whereas in human colon RKO cells putrescine induces cJun and cFos binding to AP-1 sites (364). In quiescent liver, when Matla is highly expressed, E2F1, E2F3, E2F4 and pocket protein p130 bind to the rat Mat2a promoter, whereas during regeneration $\mathrm{Sp} 1$ binds when the gene is already being transcribed and p130 is displaced at the G1-S transition (317). The lncRNA PARTICLE shares the same genomic localization than $M A T 2 A$ and a $\mathrm{CpG}$ island, whose methylation level changes after irradiation (266). PARTICLE levels increase in irradiated tumor and noncancerous cell lines and, in this context, recruits G9a histone methyltransferase, Myb and SUZ12 (a subunit of Polycomb repressive complex PRC2) to silence the MAT2A promoter.

Studies on the $M A T 2 B$ promoter are more limited, but upregulation upon binding of Sp1 and Sp3 factors to one Sp1 site have been described (Fig. 2C)(204). Later, when the existence of several $M A T 2 B$ splicing forms was reported, it was found that NFאB 
Pajares and Pérez-Sala

and AP-1 binding only induces V1 expression (404). All these elements/factors offer additional options for regulation by oxidative stress.

Expression of MAT1A and MAT2A is post-transcriptionally regulated in fetal liver development and HCC (378) (Fig. 2A). During hepatocyte dedifferentiation, levels of AU-rich RNA binding factor 1 (AUF1) increase, favoring binding to the 3'-UTR of MAT1A mRNA and promoting its degradation (378). Conversely, stabilization of MAT2A mRNA occurs upon Hu antigen R (HuR) binding to AU-rich elements in its 3'UTR. However, during liver development or AdoMet treatment of hepatoma cells the methylated-HuR levels increase and their binding to $M A T 2 A$ mRNA induces its destabilization. Downregulation of expression by microRNA binding to the 3'-UTR of target mRNAs often occurs in carcinogenesis. Among them, miR-22 and miR-29b reduce rat Matla expression (184), and miR-664, miR-485-3p and miR-495 that of human MAT1A during hepatocarcinogenesis (405).

The three mammalian MAT isoenzymes were named consecutively, according to their order of elution from phenyl Sepharose columns. Their oligomeric composition corresponds to homotetramers (MAT I) and homodimers (MAT III) of MAT $\alpha 1$ catalytic subunits or hetero-trimers (MAT II) including a MAT $\alpha 2$ catalytic dimer and one MAT $\beta$ regulatory subunit $(115,261)$ (Fig. 3). MAT isoenzymes are paradigmatic cases of redox regulation, due to the differential effects exerted by the GSH/GSSG and NADPH/NADP ${ }^{+}$ratios. The former modulates MAT I/III inhibition by GSSG $(234,276)$, whereas a decrease of the latter favors $\mathrm{NADP}^{+}$binding to MAT $\beta$ subunits, subsequently increasing their affinity for MAT $\alpha 2$ dimers (115). Thus, a connection between transsulfuration, glutathione synthesis, the polyol and pentose phosphate pathways and $\mathrm{NAD}^{+}$synthesis is provided. 
Pajares and Pérez-Sala

Both MAT $\alpha 1$ and MAT $\alpha 2$ subunits are organized in three domains that contribute to a large flat hydrophobic surface through which two monomers interact to constitute the homodimer (116,334) (Fig. 4). Two non-consecutive segments of the main chain make each domain and two active sites, opposite one to another, exist per dimer at the interface between MAT $\alpha$ subunits. Each monomer contributes part of the residues for each active site, and hence dimers are the minimum catalytic unit. The MAT $\alpha 1$ central domain contains 5 of the 10 cysteines of this subunit and is involved in the interaction between dimers in MAT I (116,325). In contrast, MAT 22 only contains 6 cysteines, 3 of them in the central domain, and MAT $\beta$ includes two domains with short-chain dehydrogenase/reductase (SDR) homology that bind $\mathrm{NADP}^{+}$and nucleotide-sugar substrates, respectively (334) (Fig. 5).

AdoMet synthesis requires methionine, ATP, $\mathrm{K}^{+}$and $\mathrm{Mg}^{2+}$, and hence production of the methyl donor depends on the ingestion of methionine and ATP availability. Intracellular ATP (millimolar) and methionine (micromolar) concentrations differ notably, the availability of the latter becoming the limiting factor. The reaction catalyzed by MATs follows a $\mathrm{S}_{\mathrm{N}} 2$ mechanism and involves two steps (reviewed in $(228,277))$. First, AdoMet synthesis by addition of the adenosyl moiety of ATP to the sulfur on the methionine lateral chain and, second, triphosphate hydrolysis to obtain the energy required for AdoMet to leave the active site. MAT isoenzymes differ in their affinities for methionine, which are 3.3-20 $\mu \mathrm{M}$ for MAT II, $80 \mu \mathrm{M}$ for MAT $\alpha 2$ homooligomers, $120 \mu \mathrm{M}$ for MAT I and $\sim 1 \mathrm{mM}$ for MAT III; their $\mathrm{V}_{\max }$ follow the opposite pattern (reviewed in (277)). Thus, intracellular AdoMet levels depend on the MAT isoenzyme expressed, a fact that becomes crucial for the liver, where $\sim 50 \%$ of the ingested methionine is metabolized $(3,416)$. Estimations of the rodent hepatic methionine content under standard diets range between $120-220 \mathrm{nmol} / \mathrm{g}$ of tissue $(88$ - 
Pajares and Pérez-Sala

$120 \mu \mathrm{M})(71,72,100)$, conditions under which MAT III would not achieve its maximal capacity for AdoMet production.

Classically MATs were considered cytosolic proteins, and hence AdoMet synthesis a cytosolic process (Fig. 6). This view was further supported by the discovery of a transporter that imports AdoMet to cover mitochondrial needs ( $\sim 30 \%$ of hepatic AdoMet) (2,140). Additionally, the small size of AdoMet was assumed to allow its transport through the nuclear pores to support nuclear methylations. However, recent reports demonstrated the existence of small nuclear pools of MAT $\alpha 1$, MAT $\alpha 2$ and MAT $\beta(168,311,398)$, suggesting that at least part of the nuclear AdoMet required may be generated inside this compartment. Interestingly, rat MAT $\alpha 1$ displays nuclear localization in those tissues/cell types with low Matla expression (311). This same study identified two overlapping areas in the rat MAT $\alpha 1 \mathrm{C}$-terminus involved in its cytoplasmic retention and nuclear localization. Basic residues delimiting these areas are mostly conserved in human MAT $\alpha 1$, suggesting preservation of their role. Moreover, hepatic nuclear fractions contain MAT $\alpha 1$ monomers and active MAT I tetramers and, among the epigenetic methylations analyzed, increased histone 3 K27 trimethylation (H3K27me3) correlates with nuclear accumulation (311). These data suggest that the nuclear enzyme sustains specific modifications, a fact later confirmed when the ability of nuclear MAT II to synthesize AdoMet for histone methylation was also demonstrated (168).

\subsection{Selected examples of genes and enzymes of transmethylation}

There are dozens of AdoMet-dependent methyltransferases that, among others, synthesize small molecules such as neurotransmitters or phospholipids, catalyze detoxification reactions (section 6) or introduce epigenetic modifications (section 4). Therefore, a complete overview of all these reactions is not feasible, and hence this 
Pajares and Pérez-Sala

section will deal with three small molecule methyltransferases that are the main consumers of hepatic AdoMet. These are glycine (GNMT), phosphatidylethanolamine (PEMT) and guanidinoacetate (GAMT) N-methyltransferases.

Main characteristics of GNMT, PEMT and GAMT genes are shown in Table 1. Splicing forms have been reported for these genes according to the NCBI-SNP database and a specific report on PEMT (337). Moreover, human chromosomes 2 and 13 hold GAMT pseudogenes. Expression of GAMT is ubiquitous, whereas that of GNMT and PEMT is mainly hepatic. GNMT expression is induced soon after birth, but is abolished in many cell types and HCC $(131,144)$. In several pathologies, reduced expression of these genes associates with their hypermethylation $(12,146,206)$.

The GNMT promoter contains a CCAAT box, Sp1 and NF-Y binding sites and xenobiotic response elements (XRE) (200) (Fig. 7). Several androgen response elements have been described and an androgen agonist upregulates this promoter only in androgen receptor-positive prostate cancer cells (201). Known cis-acting elements in the PEMT promoter include estrogen response elements, which upregulate hepatic expression upon estrogen binding (309), whereas Sp1 binding downregulates PEMT during adipocyte differentiation (62). Information regarding regulation of GAMT expression is limited to the identification of regulatory elements in the promoter (TRANSFAC database) and its downregulation in liver cells upon atorvastatin induction of miR-124a (296). Again, the elements/factors identified in these promoters allow redox regulation of expression.

GNMT and GAMT are mainly cytoplasmic enzymes, whereas PEMT is a transmembrane protein of the ER (Fig. 7). GNMT reaches 1-3\% of the total cytosolic protein in hepatocytes. Nevertheless, GNMT and GAMT also localize to the nucleus, where their role remains poorly explored $(158,187)$. These three methyltransferases use 
Pajares and Pérez-Sala

the $(S, S)$-AdoMet enantiomer produced by MATs to methylate their substrates, glycine, phosphatidylethanolamine and its mono- and di-methylated forms, and guanidinoacetate. Intracellular AdoMet levels are 10-90 $\mu \mathrm{M}$ depending on the cell type $(54,86)$, and hence those methyltransferases with lower affinities are more sensitive to pathological reductions in methyl donor availability. Among them, GNMT with a $\mathrm{K}_{\mathrm{m}}{ }^{\text {AdoMet }}$ between $30-200 \mu \mathrm{M}$, whereas those of GAMT and PEMT are $<20 \mu \mathrm{M}$. AdoHcy inhibits many methyltransferases, including PEMT $\left(\mathrm{K}_{\mathrm{i}}^{\text {AdoHcy }} 3.8-68 \mu \mathrm{M}\right)$, whereas GNMT is almost insensitive due to its high $\mathrm{K}_{\mathrm{i}}^{\text {AdoHcy }}(138,171)$. An additional mechanism of control for GNMT is provided by MTHF inhibition (384), allowing preservation of AdoMet when its levels are low and remethylation through MTR is needed. Retinoic acid, glucocorticoids and glucagon increase GNMT protein and activity levels $(264,319)$, but the mechanisms involved remain poorly explored.

Crystal structures show association of globular GNMT monomers into homotetramers (Fig 8). AdoMet binding to GNMT is cooperative and depends on the positive charge at the protein N-terminal $(182,268)$ (Fig. 8A). MTHF also interacts with the N-terminal, precluding AdoMet binding and access to the active site (384) (Fig. 8B). Additionally, GNMT dimers bind $4 \mathrm{~S}$ polycyclic aromatic hydrocarbon $(\mathrm{PAH})$ at a different site.

Synthesis of phosphatidylcholine (PC) by PEMT requires trimethylation of the amino group of phosphatidylethanolamine, a process that includes phosphatidyl monoand dimethyl-ethanolamine as intermediates (Fig. 7). This activity is very low in fetal liver, rises after birth (286), and in isolated hepatocytes is induced by several hormones $(9,47)$. Regarding PEMT organization, only topological information exists and both monomeric or dimeric association states have been proposed $(273,278)$. The model shows two AdoMet binding motifs close to the cytoplasmic side of the membrane (338). 
Pajares and Pérez-Sala

Monomeric GAMT has a single domain structure, where the guanidinoacetate and AdoMet binding sites are located, and a Rossman fold for putative $\mathrm{NAD}^{+} / \mathrm{NADP}^{+}$ binding (180) (Fig. 8C). GAMT activity depends on three sulfhydryl groups, two of which cannot be modified after GSSG inhibition (269). This effect, together with $\mathrm{NAD}^{+} / \mathrm{NADP}^{+}$binding, adds to the redox regulation of transsulfuration.

\subsection{Homocysteine synthesis and catabolism}

Genes involved in Hcy synthesis and utilization, $A H C Y, C B S$ and $C T H$ constitute the third group of genes whose main characteristics are displayed in Table 1. Splicing forms have been reported for all these genes, as well as the $A H C Y$ paralogs $A H C Y L 1$ and $A H C Y L 2$ (77). $A H C Y, C B S$ and $C T H$ display ubiquitous expression, both in human and rodents (54). Promoters of these genes also present elements for binding of redox-sensitive factors. The $A H C Y$ promoter contains POU2F1 and AP-1 binding sites that during genotoxic stress bind Oct-1 and cJun/ATF2, respectively $(129,167)$ (Fig. 9).

The $C B S$ 5'-UTR presents no TATA box, but a CAAT sequence, a variety of cis-acting elements and two promoters (Fig. 9). The use of alternative non-coding exons generates mRNAs that differ in their 5 '-end $(16,52)$. The most abundant forms are named $C B S-1 a$ and $C B S-1 b$ and are detected in adult and fetal tissues (16), whereas in hepatoma cells the main transcribed form uses the $-1 b$ promoter (108). Functional binding for Sp1/Sp3, NF-Y and NF-1 like factors to the CAAT-box in the $-1 b$ promoter was demonstrated, as well as USF-1 binding to the E-box (107-109,226). Sp1 and Sp3 activate both $-1 a$ and $-1 b$ promoters, but only the $-1 b$ promoter is synergistically upregulated (226). Tissue specific regulation of $C B S$ may involve binding of Kruppellike factors to both promoters (226). Livers of diabetic animals and glucocorticoidtreated hepatoma cells show increased $C B S$ mRNA, protein and activity levels; insulin 
Pajares and Pérez-Sala

reverts these effects reducing the $-1 b$ promoter activity (306). Conversely, glucocorticoids upregulate Sp3 expression in hepatocytes, in turn, downregulating $C B S$ $(421)$.

The $C T H$ promoter presents functional $\mathrm{Sp} 1, \mathrm{NF \kappa B}$ and TCF/LEF binding sites and ATF4 binding to a cis-intronic site $(95,254,392,403)$ (Fig. 9). Upregulation of $C T H$ expression by bile acids in the liver, $17, \beta$-estradiol in the myocardium and the $\mathrm{PI} 3 \mathrm{~K} / \mathrm{AKT}$ pathway in hepatoma cells involve a farnesoid responsive element (FXR) and Sp1 binding $(308,391,414)$. In contrast, CTH downregulation in Huntington's disease and HHcy involves Sp1 binding and promoter hypermethylation, respectively $(208,282)$. Post-transcriptional downregulation of $C T H$ expression in macrophages and cardiomyocytes implicates miR-216a and the miR-30 family, respectively $(114,336)$.

Homotetrameric AHCY catalyzes the reversible hydrolysis of AdoHcy producing Hcy and adenosine (70); in vitro, the synthesis of AdoHcy is thermodynamically favored (Fig. 10A). AHCY is mainly a cytoplasmic enzyme, although its nuclear localization has been linked to AdoHcy elimination during cap methylation (304). This protein contains a motif for the tight binding of one $\mathrm{NAD}^{+} / \mathrm{NADH}$ molecule per subunit (370). During AdoHcy hydrolysis the $\mathrm{NAD}^{+}$ cofactor is initially reduced, but later oxidized to produce adenosine (279). AHCY has high affinity for copper $\left(\mathrm{K}_{\mathrm{i}} 14 \mathrm{nM}\right)$, its binding inducing $\mathrm{NAD}^{+}$dissociation and enzyme inhibition (209). Adenosine inhibits AHCY, whereas cAMP competes with the nucleoside and counteracts its effects on AdoHcy hydrolysis (177). Each AHCY monomer includes two domains connected by helical segments that are involved in intersubunit interactions (Fig. 10A). The main chain extends from the catalytic domain, through the helical segments, into the cofactor's domain and returns to complete the catalytic domain (371). Structural data also show high similarity between the folding 
Pajares and Pérez-Sala

patterns of AHCY and GNMT catalytic domains. The $\mathrm{NAD}^{+}$cofactor binds at a cleft between domains, through residues belonging to different monomers. This organization places the four cofactor domains at the center of the tetramer's structure and the catalytic domains at the periphery (370).

Homotetrameric CBS synthesizes cystathionine by condensation of Hcy with serine, although substitution of the latter by cysteine produces $\mathrm{H}_{2} \mathrm{~S}(15,160)$ (Fig. 10B). Additionally, CBS can use cysteine and $\mathrm{H}_{2} \mathrm{O}$ or two cysteine molecules to synthesize $\mathrm{H}_{2} \mathrm{~S}$, together with serine and lanthionine, respectively (160). The enzyme is mainly cytoplasmic, but nuclear CBS has also been detected. Its affinity for sulfur substrates is low, requires pyridoxal phosphate as cofactor (121), is allosterically activated by AdoMet and binds a heme group (101,360). The presence of this group connects with iron metabolism and allows redox sensing, but heme loss makes CBS insensitive to redox stress (360). CO and NO binding to the ferrous state of the heme group inhibit CBS (379). However, enzyme activity is increased under oxidative conditions by C346 glutathionylation, a process that putatively involves initial oxidation to sulfenic acid and enzymes such as glutaredoxin (265). Nevertheless, no additional redox sensing is provided by the presence of a CXXC oxidoreductase motif (360). The crystal structure shows a two-domain organization (Fig. 10B). The catalytic N-terminal domain holds the vitamin $\mathrm{B}_{6}$ and heme binding sites, whereas AdoMet binding occurs at the regulatory $\mathrm{C}$-terminal domain, whose structure has been identified in a large variety of proteins allowing their AdoMet-dependent regulation (174).

Homotetrameric $\mathrm{CTH}$ hydrolyzes cystathionine into cysteine in a reaction that requires vitamin $\mathrm{B}_{6}$ as cofactor (30), but it also uses cysteine and/or Hcy as substrates in reactions where $\mathrm{H}_{2} \mathrm{~S}$ is produced $(15,160,174)$ (Fig. 10C). CTH is a cytoplasmic enzyme whose activity is enhanced at birth, correlating with increased glutathione 
Pajares and Pérez-Sala

needs, and that exhibits low affinity for its sulfur substrates (347). Crystal structures show a two-domain structure for the monomer, where vitamin $\mathrm{B}_{6}$ binds to the larger domain through residues contributed by two subunits (353). Two loops at either side of the $\mathrm{B}_{6}$ binding cleft approach each other in the presence of the vitamin leading to the closed conformation, where the N-terminal domain moves towards the contiguous monomer to become part of the adjacent $\mathrm{B}_{6}$ binding site. This movement increases the hydrophobic interactions among monomers in the CTH tetramer (353). 
Pajares and Pérez-Sala

\section{REGULATION OF TRANSSULFURATION}

This section is intended to summarize the main regulatory mechanisms of transsulfuration that involve, among others, the diet and protein-protein interactions.

\subsection{Regulation of transsulfuration by the diet}

The transsulfuration pathway requires several essential nutrients for its adequate function and regulation, among them, methionine, vitamins and cations. Therefore, the route is highly dependent on the dietary intake of these nutrients that may show a high variability depending on cultural, geographic and economic reasons. The protein source used determines the amount of methionine ingested, since its content tends to be lower in vegetables and digestion of plant proteins is less efficient (145). Protein rich diets activate FXR, a nutrient sensing nuclear receptor, upregulating hepatic amino acid catabolism and Mat1a, Ahcy and Cth expression (241) (Fig. 11). In contrast, methionine restriction extends lifespan in several organisms including rodents (271), an effect ascribed to reduced mitochondrial production of reactive oxygen species (ROS) due to inhibition of respiratory complexes I and III, rather than to increased antioxidant capacity $(326,327)$. In fact, rats fed methionine-restricted diets show no alteration of GSH levels in most tissues, but reduced hepatic and renal concentrations (312); increased GSH plasmatic levels seem to rely on its hepatic efflux. Conversely, hepatic GSH increases in mice fed methionine-supplemented diets, whereas both deficiency and supplementation enhance GSSG levels (4). Overall, both nutritional regimes reduce the GSH/GSSG ratio. Longevity induced by nutritional restriction regimes in several experimental models also concurs with increased $\mathrm{H}_{2} \mathrm{~S}$ production through transsulfuration that seems tissue/cell specific (136). Moreover, in dwarf mice models of longevity reduced levels of growth and thyroid hormones upregulate hepatic Cth expression, $\mathrm{CTH}$ and $\mathrm{CBS}$ protein levels and $\mathrm{H}_{2} \mathrm{~S}$ synthesis (137). Lifespan can also be 
Pajares and Pérez-Sala

extended by tissue-specific downregulation of AHCY through elimination of two AHCY-like proteins in flies (280), whereas its shortening is detected in mice lacking methionine sulfoxide reductase A (MsrA), which reverts oxidation at methionine residues that occurs during aging (257).

Some data suggest that methionine availability may affect its distribution between protein and AdoMet synthesis differentially. For instance, methioninesupplemented cultures of vascular endothelial and hepatoma cells show induction of elongation factors (EF-1 $\alpha, \mathrm{EF}-1 \beta$ and $\mathrm{EF}-1 \delta)$ and $M A T 2 B$ expression, respectively $(50,118)$. In contrast, restriction increases expression of the three $M A T$ genes in hepatoma, whereas only $M A T 2 A$ and $M A T 2 B$ are upregulated in kidney Cos7 cells $(118,233)$. Nevertheless, more work is required in this area to fully understand the putative connections.

In rodents, methionine restriction downregulates hepatic expression of Ahcy, Cbs and Gnmt (4) (Fig. 11). In the long-term, the liver and inguinal white adipose tissues are the main targets for expression changes induced by this diet (110). Hepatic alterations include upregulation of glutathione and xenobiotic metabolism by cytochrome P450, as well as up- (e.g. Myc) or down-regulation (e.g. NFkB, Jun) of several redox-regulated transcription factors. Feeding of either methionine deficient or supplemented diets also increases hepatic SREBP1, CEBP $\beta$ and CEBP $\alpha$ protein levels, whereas supplementation reduces PPAR $\alpha$ content (4). Whether such effects occur in other organs or if dietary restrictions in other amino acids could elicit these changes was not studied. Maternal methionine ingestion during pregnancy also affects expression in the offspring, supplementation upregulating transsulfuration genes, GCLC and GSR $(151,375)$. Methionine deficiency and excess contribute to genomic instability and metabolic alterations that are related to cancer prognosis $(38,294)$. Mice fed either type 
Pajares and Pérez-Sala

of diet show decreased hepatic methionine concentrations, together with increased content of AdoMet, AdoHcy and Hcy (4). In addition, levels of 4-hydroxynonenal (HNE) protein adducts are increased, indicating enhanced lipid peroxidation and oxidative stress.

Methionine restriction also alters ATP synthesis through its effects on global transcriptional and translational programs (426). In this setting, oxidative phosphorylation is favored, allowing the best energy yield. AMPK, the main energy sensor, prevents significant changes in ATP concentrations (43), a task achieved in part by recycling adenosine derived from transsulfuration. Furthermore, the connection with ATP synthesis is also supported by AMPK activation through allosteric binding of AMP to a CBS domain in its $\gamma$-subunit (399). High AdoMet levels preclude AMPK activation by phosphorylation of its $\alpha$-subunit, in turn, preventing HuR transport to the cytoplasm for mRNA stabilization, including that of MAT2A (237).

In many cancer cells the switch towards glycolysis (Warburg effect) reduces the yield of ATP produced and, if methionine is restricted, initial steps of transsulfuration become dependent on the contribution of serine to de novo purine synthesis and, subsequently to ATP pools (227). Crossregulation between pathways is further highlighted by the depletion of ATP and $\mathrm{NAD}^{+}$pools caused by MAT 1 overexpression in $\mathrm{CHO}$ cells (324) and the regulation of autophagy by methylation and $\mathrm{H}_{2} \mathrm{~S}$ (355,357). Methionine controls yeast autophagy through the methylation status of the protein phosphatase 2A (PP2A) catalytic subunit; increased AdoMet availability favors PP2A methylation and then Npr2p dephosphorylation and inhibition of nonnitrogen-starvation-induced autophagy. Moreover, addition of a $\mathrm{H}_{2} \mathrm{~S}$ precursor to cultured Werner syndrome's fibroblasts also inhibits mTOR activation (357). 
Pajares and Pérez-Sala

Micronutrients required by several steps of transsulfuration and some reactions refeeding the pathway are obtained from the diet (Fig. 11). Marginal vitamin $\mathrm{B}_{6}$ deficiencies seem quite common worldwide and lead to increased plasma cystathionine and serine levels (69). Effects of $\mathrm{B}_{6}$ deficient diets vary from increased levels of plasma Hcy, hepatic Hcy and cysteine, together with decreased AdoMet content and impaired folate metabolism to small effects in the flux through transsulfuration and one carbon metabolism $(231,314)$. In hepatoma cells, this deficiency increases cystathionine, cysteine and GSH levels (68), whereas production of $\mathrm{H}_{2} \mathrm{~S}$ remains unchanged (76). Vitamin $\mathrm{B}_{12}$ and folate also influence transsulfuration, mainly through their roles in Hcy remethylation, where maintenance of MTR activity requires the reductive regeneration of $\mathrm{B}_{12}$ catalyzed by methionine synthase reductase (MTRR). Additionally, low folate levels increase MAT $\alpha 2$ acetylation, reducing its stability (411). Rats fed nicotinamide supplements show increased hepatic MAT and CBS activities (102), together with Nnmt and Bhmt promoter hypomethylation and Cbs promoter hypermethylation (207). Moreover, nicotinamide and 1-methylnicotinamide, the product of nicotinamide $\mathrm{N}$ methyltransferase (NNMT), are neuroprotective against Hcy toxicity in cell culture (340).

Transsulfuration is also altered in obesity, where increased plasma cysteine levels are detected, putatively reflecting anomalous $\mathrm{H}_{2} \mathrm{~S}$ production that subsequently modifies glucose and lipid homeostasis (45). Leptin-mediated weight loss of ob/ob mice upregulates hepatic Mat2a, but downregulates Ahcy, Gamt and Bhmt (335). Experiments with $\mathrm{Pemt}^{-/}$mice fed high fat diets demonstrated the need of sufficient choline, rather than PC, to avoid obesity (150). Effects on transsulfuration also expand to dietary components/additives. The antioxidant resveratrol induces $M A T 2 B-\mathrm{V} 1$ and V2 expression, favoring exchange of MAT $\beta$ interaction partners towards HuR and 
Pajares and Pérez-Sala

SIRT1 and increasing AdoMet synthesis (410). Chronic ingestion of the sweetener aspartame impairs hepatic transsulfuration and depletes glutathione (99).

\subsection{Regulation by metabolites of transsulfuration and related pathways}

Initial studies focused on AdoMet, which controls its own synthesis inhibiting MAT I and MAT II, but stimulating MAT III $(34,202)$. High AdoMet levels activate CBS and GNMT and inhibit methylenetetrahydrofolate reductase (MTHFR) and MTR activities $(101,153)$, favoring Hcy catabolism versus remethylation, an effect to which the different $\mathrm{K}_{\mathrm{m}}{ }^{\mathrm{Hcy}}$ of each enzyme further contribute. High AdoMet concentrations also preclude upregulation of $M A T 2 A$ expression in hepatoma cells (233) and downregulate BHMT expression (272), effects that are mimicked by MTA produced during polyamine synthesis. Recent evidences also demonstrate that AdoMet levels regulate MAT2A splicing (287). In HEK293 cells, methionine starvation reduces AdoMet levels increasing the occupancy of a conserved hairpin (hp1) at the MAT2A 3'-UTR by the U6 snRNA $\mathrm{m}^{6} \mathrm{~A}$ methyltransferase METTL16. The authors hypothesized that a longer dwell-time of METTL16 on hp1, not its methylation, dictates removal of the retained intron 8, enhancing $M A T 2 A$ expression. These data establish a new link for transsulfuration, this time with the splicing machinery.

Methyltransferases are inhibited by AdoHcy and MTA (60), but AdoHcy is also able to counteract AdoMet inhibition of MTHFR. Hence, MTHF production increases, inhibiting GNMT to boost AdoMet availability (412). Adenosine and its derivatives, such as 3-deazaadenosine, inhibit AHCY leading to increased AdoHcy levels and a reduced methylation index (57), a property that prompted an extensive exploration of their potential use as antivirals. Hcy controls transsulfuration and protein synthesis, inducing $C T H$ promoter hypermethylation and EF-1 $\delta$ expression in macrophages and vascular endothelial cells, respectively $(50,208)$. Altogether a crossregulation between 
Pajares and Pérez-Sala

the folate cycle, polyamine and protein synthesis and transsulfuration is established and further complicated by effects of transsulfuration metabolites on their own pathway. Additional effects will be analyzed in section 5.

\subsection{New players identified through protein-protein interactions}

Protein-protein interactions in transsulfuration are starting to be explored and, in fact, few studies have dealt with the effects of the known interactions. Databases such as IntAct or BioGRID contain results from high-throughput analysis of interest for this review, although many of the reported interactions have not been verified by additional procedures nor their functional role elucidated. Therefore, we will concentrate on a few protein targets with a known impact on transsulfuration.

Among MATs, the available information includes the already mentioned interaction of MAT $\alpha 2$ with MAT $\beta$, leading to the MAT II heterotrimer, for which different interacting surfaces have been reported $(115,203,261)$. Some studies also mentioned the interaction of MAT $\beta$ with MAT $\alpha 1$, but no further investigation has been carried out in this line. In colon RKO and hepatic HepG2 cancer cells increased levels of both MAT $\beta-\mathrm{V} 1$ and V2 splicing forms are detected, besides their interaction with HuR (Fig 12). This interaction enhances HuR cytoplasmic localization, subsequently increasing cyclin D1 and cyclin A expression (398). SIRT1 also binds to MAT $\beta$ in this context, the MAT $\beta / \mathrm{HuR} / \mathrm{SIRT} 1$ interaction enhancing the stability of the three proteins (410). The antioxidant resveratrol favors this tripartite interaction by inducing MAT $\beta$ V1 and V2 expression and weakening MAT $\alpha 2_{2} / \mathrm{MAT} \beta$ binding (410). MAT $\beta$-V1 and V2 variants also interact with GIT1, MEK1 and ERK2 and the MAT $\beta /$ GIT1 interaction is required to activate MEK1/ERK to favor cell growth in several types of cancer (288). Both MAT $\beta$ forms joined GIT1 to interact with B-Raf and cRaf, but only MAT $\beta$ interacts with Ras and contributes to protein stabilization (289). This array of 
Pajares and Pérez-Sala

interactions further contributes to cell growth through the Ras/Raf/MEK/ERK signaling cascade in transformed cells.

Using tandem affinity purification followed by mass spectrometry and immunoprecipitation MAT 22 was found as a nuclear interaction target for the MafK oncoprotein in mouse X63/0 plasmacytome cells (168). This protein is a small Maf of the AP-1 family that forms either homodimers to repress antioxidant response elements (AREs) or heterodimers that act as activators or repressors of these elements. MAT $\alpha 2$ behaves as MafK corepressor to reduce heme oxygenase-1 $(\mathrm{HO}-1)$ gene transcription in hepatoma Hepa-1 cells and immortalized mouse embryonic fibroblasts (iMEF) $(168,170)$, a process in which the decrease of H3K4me2 and H3K9me2 marks on the gene enhancers is involved. Additionally, this same study identified more than a hundred nuclear MAT $\alpha 2$ interaction targets in MEL cells and, among them, MAT $\beta$, histone methyltransferases (G9a, Ehmt1 and ALL1/KMT2A), several subunits of the Polycomb repressive complex 1 (PRC1), members of the chromatin remodeling complex Swi/Snf and the PARP1 complex. Nuclear MAT 2 also interacts with methyltransferases SETDB1 and Suv39h1 in iMEF cells, where the MATa2/SETDB1 oligomer is recruited to a Maf recognition element on the $C O X-2$ promoter for its repression (170).

The oncogene PDRG1 interacts with MAT $\alpha 1$ and MAT $\alpha 2$ homo-oligomers and MAT II, reducing their AdoMet synthesizing activity and excluding MAT $\beta$ from the heterotrimer (291). Interestingly, the PDRG1/MAT $\alpha 1$ interaction seems restricted to the nucleus, where both proteins accumulate in acute liver injury and $\operatorname{HCC}(71,291,405)$. These are two pathologies in which expression of PDRG1 and MAT2A increase, while the MAT1A/MAT2A switch is produced together with a reduction in global AdoMet levels. In normal liver, nuclear MAT $\alpha 1$ also interacts with the Max network 
Pajares and Pérez-Sala

transcriptional repressor Mnt (408). This MAT $\alpha 1$ interaction changes towards cMyc, cMaf and MafG in cholestatic and cholangiocarcinoma tissues, where the levels of these three proteins increase. These complexes bind to the E-boxes in MAT1A, $c-M Y C, c-$ $M A F$ and $M A F G$ promoters to regulate their expression. Max is also an interaction target for MAT $\alpha 1$, but this complex remains unaffected in cholestasis and cholangiocarcinoma (408).

The nucleocytoplasmic transport of MATs has not been specifically studied and neither PredictNLS or NetNES identified consensus localization or export signals in their sequences. Leptomycin inhibition of the CRM1 exportin did not affect nucleocytoplasmic distribution of rat MAT 1 (311), despite the reported CRM1/XPO1 interaction with MAT in ancient metazoan (387). This result correlates with the low scores given by NetNES to the V360-L367 sequence in MAT $\alpha 1$ and I305 in MAT $\beta$, and the negative scores for MAT $\alpha 2$.

GNMT levels decrease drastically in HCC, thus impeding its protein-protein interactions. Among them, abolition of the GNMT/PREX2 interaction precludes ubiquitination-mediated proteasomal degradation of this PTEN inhibitor, leading to AKT activation and anomalous proliferation (205). Elimination of this interaction is probably a main contributor to HCC in $G n m t^{-/-}$null mice. GNMT, as the PAH receptor, interacts with the aryl hydrocarbon receptor nuclear translocator (ARNT/HIF-1 $\beta$ ) in CHO cells (349). However, upon binding of benzo(a)pyrene, GNMT dissociates from this oligomer and translocates to the nucleus for the activation of an XRE in the CYP1A1 promoter.

Hepatic CTH and BHMT interact with cytoplasmic high-mobility group box 1 (HMGB1) in ischemia/reperfusion (418). This protein contributes to nucleosome stability, facilitates transcription and, in the extracellular space, interacts with advanced 
Pajares and Pérez-Sala

glycation end products and toll-like receptors stimulating the release of proinflammatory cytokines and activating the redox-regulated JNK and NFאB pathways in response to organ damage $(21,373)$. Moreover, HMGB1 binding to beclin1 promotes autophagy (358), whereas CTH and BHMT synthesize sulfide and methionine, respectively, that are inhibitory $(355,357)$. Altogether, exchange of HMGB1 proteinprotein interactions suggests a role in the control of autophagy.

\subsection{Regulatory mechanisms through post-translational modifications}

Human transsulfuration enzymes have been identified in high-throughput analysis of post-translational modifications (PTMs). Most of them present different degrees of phosphorylation, ubiquitination, acetylation and methylation, although some cases of succinylation, sumoylation or O-linked $\mathrm{N}$-acetylglucosamine incorporation (OGlcNAc) are also reported. The initiator methionine is lost in MAT $\beta$ (203), GAMT (23), PEMT, GNMT, AHCY (123) and CBS. Nevertheless, few are the cases in which the functional role of these modifications is known.

The three MAT subunits present several PTMs that for MAT $\alpha 1$ are mostly related to its redox regulation. MAT I and MAT III isolated from rat liver are stable, whereas recombinant isoenzymes interconvert according to the protein concentration (251). Mutation of cysteines between positions 35 and 105 of the sequence alters the MAT I/III ratio (252), suggesting a role for these residues in isoenzyme interconversion. Rat hepatic isoenzymes contain the C35-C61 disulfide bond and in vitro studies showed that this modification precludes tetramer/dimer exchange $(235,325)$. Inhibition by GSSG and its modulation according to the GSH/GSSG ratio also indicated production of an intrasubunit disulfide (276). Chemical modification and site-directed mutagenesis showed that cysteines, and especially C121, are very important for MAT I/III activity $(252,274)$. Nitrosylation or hydroxylation at C121 erase activity. Structural information 
Pajares and Pérez-Sala

allowed identification of residues (D355, R357 and R363) involved in the acid-basecatalyzed mechanism of nitrosylation (292). Crystal structures indicate that C35 and C61 are positioned to form the intrasubunit disulfide, whereas $\mathrm{C} 121$ locates at a flexible loop controlling access to the active site $(116,117)$. These modifications may block the central domain $\beta$-sheet and the loop conformations to preclude dimer-dimer association and admittance of substrates into the catalytic site, respectively. These cysteines are preserved in human MAT $\alpha 1$, allowing the same regulatory features, a fact that is precluded for MAT 2 2, which lacks residues equivalent to C61 and C121.

PKC phosphorylation of rat MAT $\alpha 1$ occurs on $\mathrm{T} 342$ and high-throughput studies found the equivalent modification in its human counterpart. This phosphorylation alters MAT I/III activity and weakens the monomer-monomer interactions (275). Location of this residue close to the areas involved in subcellular localization suggests a role for this phosphorylation in MAT $\alpha 1$ distribution that deserves investigation. MAT $\alpha 2$ and MAT $\beta$ phosphorylation in hepatic stellate cells enhances their stability. However, while MEK modifies both subunits, ERK1/2 or BRaf only phosphorylate MAT $\beta$ (305).

P300 acetylates MAT $\alpha 2$ on K81, favoring ubiquitination by the ubiquitin ligase UBR4 and its targeting for proteasomal degradation (411). MAT $\alpha 2$ acetylation levels decrease in HCC, where deacetylation is performed by its interaction target HDAC3 (168). Nutrition influences acetylation levels, growth in folate-deficient media increasing MAT $\alpha 2$ modification and degradation (411). Ubc9 sumoylates MAT $\alpha 2$ on K340, K372 and K394 inducing its stabilization and, subsequently favoring MATa2/Bcl-2 interaction that also stabilizes Bcl-2 (363). Hence, the enhanced expression of these three proteins shown in tumor cells results in inhibition of apoptosis (365). MAT $\alpha 2$ further contributes in an AdoMet-independent manner through its 
Pajares and Pérez-Sala

binding to the $B C L 2$ promoter, upregulating its transcription. Conversely, apoptosis increases by addition of AdoMet due to reduced $U B C 9$ and MAT2A expression and the decreased MAT $\alpha 2$ availability to induce $B C L 2$ transcription.

AMPK or PKA activation promotes GNMT phosphorylation (220,385), whereas N-terminal acetylation increases its inhibition by MTHF (221). These modifications connect ATP/ADP sensing and folate availability with the regulation of AdoMet utilization. PKA and PKC phosphorylation activate hepatic PEMT in vitro $(47,380)$, whereas $\mathrm{N}$-terminal $\mathrm{N}$-glycosylation with high mannose oligosaccharides occurs in HEK293 cells (255). This last modification alters substrate specificity so that PEMT-2 hardly uses phosphatidylethanolamine, but produces PC and ether-linked PC, preferentially with polyunsaturated fatty acids (PUFAs) shorter than those synthesized by non-glycosylated PEMT-1. Regarding GAMT, only N-terminal acetylation has been reported (23).

CBS and CTH are also targets for PTMs. Carbachol stimulation of the cGMP/PKG pathway phosphorylates $\mathrm{CBS}$ on $\mathrm{S} 227$, stimulating $\mathrm{H}_{2} \mathrm{~S}$ production to control bladder tone (67). CBS glutathionylation on C346 enhances enzyme activity (265) and its sumoylation on K211 changes its localization towards the nuclear scaffold (162). For this purpose, the C-terminal domain has to interact with the sumoylation machinery, including SUMO-1. CTH phosphorylation by PKG or AKT1 on S377 regulates its function $(307,415)$. In the carotid body, the major $\mathrm{O}_{2}$ sensing organ, increased $\mathrm{O}_{2}$ levels reduce $\mathrm{H}_{2} \mathrm{~S}$ synthesis through $\mathrm{PKG}$ phosphorylation of CTH. This involves kinase stimulation by the enhanced $\mathrm{CO}$ production of $\mathrm{HO}-2$, whereas $\mathrm{NO}$ assumes PKG regulation in hypoxia. 
Pajares and Pérez-Sala

\section{EPIGENETIC MODIFICATIONS DEPENDENT ON TRANSSULFURATION}

Epigenetic modifications that depend on transsulfuration are mainly DNA and histone methylations, although the recent identification of histone homocysteinylation provides a new link (400). There are three DNA methyltransferases (DNMTs) encoded by the $D N M T 1, D N M T 3 A$ and $D N M T 3 B$ genes, but more histone methyltransferases are known. Thus, we will focus mainly on EZH2, encoding the Enhancer of Zeste Homologue 2 histone methyltransferase catalyzing H3K27me3.

\subsection{Expression of epigenetic methyltransferases}

Epigenetic methyltransferases present ubiquitous expression, although DNMT3B and $E Z H 2$ exhibit higher levels in testis $(53,315)$. All of them have splicing forms (Table 1) and DNMT1 and DNMT3B genes locate close to GAMT and $A H C Y$ on human chromosomes 19 and 20, respectively. DNMT genes lack canonical TATA boxes and present several promoter sequences $(24,156)$. A number of AP-1, E2F, TCF, Sp1, Sp3 and STAT3 SIE/GAS binding sites, some of which allow redox regulation, have been recognized in DNMT1 and their functionality analyzed (Fig. 13). In HepG2 cells, ursolic acid decreases Sp1 expression, subsequently reducing DNMT1 expression and phosphorylation (413). Moreover, DNMT3A and DNMT3B expression is upregulated by binding of Sp1 and Sp3 factors to Sp1 binding sites on their 5'-UTR sequences (156). DNMTs expression is increased by ROS, but inhibited by Nrf2 activators that also reduce their activity $(143,310)$. The diet is also a factor influencing DNA methylation, thus nicotinamide supplements reduce this modification, while increasing MAT activity (207). Importantly, the methyl donor composition of the maternal diet (e.g. methionine content) affects DNA methylation levels in the infants and, among them, those of metabolic genes (283). In fact, maternal methionine supplementation upregulates expression of DNMTs in the offspring (151). 
Pajares and Pérez-Sala

The EZH2 promoter presents a variety of regulatory elements and functional binding has been reported in several settings, unveiling a complex regulation. Among others, EZH2 repression is regulated by NFIB and p53 (297,359), whereas its activation is induced by cRel, RelA and RelB (263) (Fig. 13). Additionally, EZH2 transcript levels in HCC are reduced by binding of several microRNAs to its 3'-UTR, including miR26a, miR-214 and miR-98 $(397,417,425)$. Conversely, the lncRNA ANCR mediates EZH2 degradation in breast cancer (211).

\subsection{Main features of epigenetic methyltransferases}

Epigenetic methyltransferases are nuclear proteins that consume a modest amount of AdoMet compared to PEMT, GNMT and GAMT. Their $\mathrm{K}_{\mathrm{m}}{ }^{\text {AdoMet }}$ are $1.5 \mu \mathrm{M}$ for EZH2 and 3-14 $\mu \mathrm{M}$ for DNMTs, therefore only severe pathological decreases in AdoMet content would alter their function. Nevertheless, their different affinities for AdoMet make DNMTs more susceptible to its changes than EZH2.

DNMTs transfer a methyl group from AdoMet to the C5 on the cytosine located at $\mathrm{CpG}$ dinucleotides, which are mostly methylated in the genome (Fig. 13). CpG islands occupy $60 \%$ of human promoter sequences and variability in their methylation status determines expression of the corresponding gene, as this modification interferes with binding of transcription factors. DNMT structures differ mainly in their N-terminal regulatory domain. Homodimeric DNMT1 is the most abundant and guarantees maintenance of DNA methylation patterns during replication, allowing their transfer to the next generation (22). In contrast, homodimeric DNMT3A and DNMT3B are involved in de novo methylation (270). Their activity is modulated by DNMT3L homodimers, a third member of this family lacking methyltransferase activity, and together constitute heterotetrameric enzymes (341). Increased levels of DMNT3L induce the release of DNMT3A from the heterochromatin (159). Additionally, DNMT1 
Pajares and Pérez-Sala

forms hetero-oligomers with de novo methyltransferases DNMT3A and DNMT3B through their N-terminals (172). This fact, together with spreading of methylation over the genome upon coexpression of these DNMTs, suggests their cooperation to control gene expression.

DNMT1 is involved in a variety of protein-protein interactions which are modulated by its PTMs in a position-specific manner. Therefore, only some examples will be mentioned. CDK5 phosphorylation on S154, interaction with the ubiquitin ligase UHRF1, multiple sumoylation by Ubc9 or deacetylation at residues K1349 and K1415 by SIRT1 activate DNMT1 $(105,199,290,300)$. Increased stability occurs by interaction with the Hsp90 chaperone, deubiquitination by USP7, or by reducing/precluding methylation through AKT1 phosphorylation on S143 and interaction with $\beta$-catenin $(92,299,344,423)$. Conversely, DNMT1 proteasomal degradation is promoted by SET7 methylation on K142 or its ubiquitination as a consequence of the lack of interaction with hyperacetylated Hsp90 (298,423). Additionally, the presence of certain PTMs in DNMT1 may be required for protein-protein interactions. For example, 14-3-3 binds S143 phosphorylated DNMT1 after replication, reducing its activity, and the MBT domain of PHF20L1 interacts with K142 monomethylated DNMT1, precluding its ubiquitination and degradation $(93,94)$. Furthermore, interaction with the nuclear import regulator BRAP2 impedes DNMT1 nuclear localization, whereas the mismatch repair MSH2/MSH6 heterodimer recruits the methyltransferase to DNA damaged sites to reduce transcription at these locations $(81,97)$.

DNMT3A and DNMT3B are targets of several PTMs that modify their activity. Among them, phosphorylation by ERK1/ERK2 blocks recruitment of DMNT3A to the Sox9 promoter (189), S386 and S389 phosphorylation by CK2 reduces methyltransferase activity and binding to the heterochromatin preventing methylation of 
Pajares and Pérez-Sala

Alu or SINE repeats (74). Citrullination by peptidylarginine deiminase 4 stabilizes DNMT3A and seems key for p21 promoter methylation (75). Sumoylation disrupts DNMT3A interaction with HDAC1 and HDAC2, repressing transcription (134). DNMT3B interacts with SUMO-1 for its sumoylation (165) and with the hPc2 polycomb protein to repress fibroblast growth factor receptor 3 (173). During differentiation of human embryonic stem cells, phosphorylation of DNMTs occurs, as well as their interaction with the polymerase associated factor 1 transcriptional elongation complex (313). This complex binds to promoters of OCT4 and NANOG genes that are involved in pluripotency, putatively contributing to their silencing.

Histone methylation occurs on arginine or lysine residues, which incorporate a maximum of two or three methyl groups, respectively. EZH2 is a subunit of PRC2 that catalyzes incorporation of the $\mathrm{H} 3 \mathrm{~K} 27 \mathrm{me} 3$ repression mark, but also other protein methylations (Fig.13). Moreover, EZH2 recruits DNMTs to PRC2 and PRC3 complexes to regulate DNA methylation at EZH2-targeted promoters, a role to which interaction with the IncRNA AS1DHRS4 also contributes $(210,381)$. EZH2 interacts with EED, SUZ12 and PHF1 subunits within PRC2 (191,328,333). EED is involved in directing PRC2 to the chromatin and SUZ12 seems essential for EZH2 activity, since its absence eradicates $\mathrm{H} 3 \mathrm{~K} 27 \mathrm{me} 2$ and $\mathrm{H} 3 \mathrm{~K} 27 \mathrm{me} 3$ marks (281). In contrast, PHF1 only stimulates catalysis of the H3K27me3 mark (328). Other EZH2 interactors include: C10orf12 and C17orf96 that do not bind simultaneously within PRC2 (8); the chromodomain Y-like protein that facilitates introduction and propagation of the H3K27me3 modification by increasing PCR2 methylation activity (420); and XNP, a member of the SNF2-like family, that contributes to transcriptional regulation or DNA repair (42). 
Pajares and Pérez-Sala

EZH2 is a target for several PTMs. Among them, K348 acetylation by P300/CBP associated factor and S75 O-GlcNAcylation by interaction with OGT stabilize EZH2 $(59,388)$. Conversely, phosphorylation by JAK2, CDK5 or CDK1, the latter induced by ncRNAs ANCR and HOTAIR, promote interaction with the E3 ubiquitin ligases FBXW1 or FBW7 for EZH2 ubiquitination and degradation $(155,164,211,320)$. In some cases, an interplay between PTMs occurs for a coordinated regulation of EZH2, e.g. K348 acetylation decreases T345 and T487 phosphorylation favoring stability (388). Additionally, the presence or absence of certain PTMs promotes dissociation of EZH2 from PRC2. For example, lack of O-GlcNAcylation or Y244 phosphorylation by JAK3 disrupts the PRC2 complex, the latter allowing EZH2 interaction with Pol II and changing its role towards transcriptional activator $(59,401)$. EZH2 activity is also modulated by PTMs, e.g. S21 phosphorylation by AKT blocks histone 3 binding and T350 modification by CDK1 and CDK2 is key to maintain the repressive H3K27me3 mark at the targeted loci $(48,55)$. However, T416 phosphorylation by CDKs allows binding of NIPP1, a regulator of PP1, inhibiting dephosphorylation of EZH2 and impairing binding to polycomb target genes involved in proliferation (253). In general, EZH2 is abundant in proliferating organs and PRC2 deregulation is involved in tumorigenesis. Hence, considerable effort has been devoted to design molecules targeting the complex to control its activity. Among them, astemizol destabilizes EZH2/EED interaction reducing PRC2 methylation activity, and thus proliferation of lymphomas (181). 
Pajares and Pérez-Sala

\section{DEREGULATION OF TRANSSULFURATION IN PATHOLOGY}

Oxidative stress is commonly detected in disorders in which transsulfuration is impaired, ranging from major liver pathologies to rare diseases and sensorineural disorders. Pathological changes involve all the regulatory levels from gene expression to activity, but differences are detected among diseases (Fig. 14). Most research in this field has been carried out in liver or hepatoma cells, and hence this section will consider mainly hepatic diseases. It is necessary to remind here that hepatic cell lines (mostly hepatomas), primary hepatocytes and even iPS hepatocytes show impaired expression of those genes traditionally considered "liver specific", thus the need for overexpression (e.g. MAT $\alpha 1$ or GNMT) reported in most studies.

\subsection{Cancer and hepatocellular carcinoma}

Common features of cancer cells are their high consumption of methionine and global DNA hypomethylation. These effects can be explained by an enhanced need of the amino acid for protein synthesis in proliferating cells and alterations in AdoMet synthesis due to changes in MAT expression patterns. Impairments of transsulfuration in tumor cells have been mostly studied in HCC, therefore we will focus preferentially in this malignancy that represents $85 \%$ of all primary liver cancers.

The story about cancer and transsulfuration started with the finding that growth of tumor cells depends strictly on methionine $(88,352)$. Almost 20 years later, decreased MAT activity was found in rodent models of hepatoma together with changes in the MAT isoenzyme pattern (368). It took another 20 years to find the MAT1A/MAT2A expression switch that occurs in HCC, which involves changes in the methylation status of the gene promoters, rendering reduced MATIA together with enhanced MAT2A and MAT2B expression $(218,367)$. Changes in transsulfuration are however wider, including downregulation of GNMT (12,131), PEMT (376), AHCY (198) and CBS (12), but also 
Pajares and Pérez-Sala

of several branches of the route, including Hcy remethylation (Fig. 14). This last observation justifies why Hcy cannot substitute methionine to support tumor cell growth. Additionally, several hepatoma cells express splicing variants for MAT2B (404) and are defective in Gnmt (394) and Pemt expression (62).

Several null mice for genes in this pathway have been generated since 2000 . Most of them develop steatohepatitis and HCC due to their low $\left(\right.$ Mat1 $\left.^{-/-}\right)$or high $\left(G n m t^{-/}\right)$hepatic AdoMet levels $(215,236)$. The Pemt $t^{-/}$mouse constitutes an exception, showing severe steatosis without increased tumor incidence (150). This difference may rely on the ability to synthesize enough PC for hepatocyte function through the de novo CDP-choline pathway, whereas e.g. MAT $\alpha 2$ seems unable to produce sufficient AdoMet to reach adequate concentrations to support the wide array of AdoMetdependent reactions. Reduced MAT1A expression in HCC correlates with hypomethylation of $L I N 28 B$ and downregulation of the tumor suppressor let-7, favoring invasion and metastasis (405). Moreover, the positive crossregulation between MAT1A and PHBI expression is also lost in $\mathrm{HCC}$, where changes in the occupancy of their promoter E-boxes towards cMyc/cMaf/MafG reduce MAT $\alpha 1$ and PHB1 content (96). The role of transsulfuration in HCC is further highlighted by the impact of miRNAs levels on MATIA downregulation (miR-664, miR-485-3p, miR-405) and increased EZH2 expression (miR-124) $(405,422)$.

Pathological deregulation of transsulfuration has been associated with $\mathrm{NF \kappa B}$ and AP-1 signaling cascades that are redox-regulated. Sites for these transcription factors exist in the promoters of MAT2A, MAT2B, PEMT, GAMT and GNMT, but also in BHMT and MTRR promoters, although their role remains poorly explored. Upregulation of MAT2B, JMJD3 and NOS2 occurs upon binding to these elements, enhancing MAT $\beta$-V1 content, H3K27me3 demethylation and NO production, respectively (404). 
Pajares and Pérez-Sala

Increased Hcy concentrations trigger the proinflammatory response mediated by NFkB, leading to enhanced ROS levels (126). In HCC, increased MAT $\alpha 2$ levels would favor its corepressor role of MafK binding to ARE elements in target genes such as $\mathrm{HO}-1$ (168), therefore altering its role in iron reutilization, anti-inflammation or cytoprotection. Additional contributors to this picture are HuR and AUF1, which favor stabilization of $M A T 2 A$ and degradation of $M A T 1 A$ mRNAs, respectively (377).

Changes in expression of "liver specific" genes are mimicked by their cytosolic protein levels, although for MATs this is accompanied by enhanced MAT2A expression, MAT2B splicing forms and generation of the MAT II isoenzyme $(36,277,404)$. As a consequence, an increase in MAT oligomers with a low $\mathrm{V}_{\max }$ for AdoMet synthesis occurs. However, the picture further complicates with heterotrimers containing MAT $\beta$ splicing forms, whose impact on AdoMet synthesis remains unclear. Nevertheless, a Nterminal MAT $\beta$ truncation similar to MAT $\beta-\mathrm{V} 2$ still associates with MAT $\alpha 2$ dimers and the resulting heterotrimer exhibits a higher $\mathrm{V}_{\max }$ than MAT II in vitro (115).

Low AdoMet concentrations have been measured in several models of cancer, correlating with decreased protein and activity levels of methyltransferases consuming large amounts of AdoMet (e.g. PEMT). In contrast, modest users of AdoMet (e.g. EZH2) increase their presence in HCC correlating with tumor progression (58), whereas overexpression of NNMT occurs in a variety of cancers consuming large amounts of AdoMet to support growth (374). Moreover, low AHCY activity and the homozygous R49H mutation in this protein associate with $\mathrm{HCC}(188,348)$, putatively modifying the methylation index. Furthermore, increased NO levels due to iNOS upregulation will inhibit MAT I/III and CBS activities, through C121 nitrosylation and NO binding to the heme group, respectively $(13,379)$, but may also impair remethylation via BHMT nitrosylation as shown in cholestasis (214). In contrast, NO stimulates CTH 
Pajares and Pérez-Sala

transcription, and thus, $\mathrm{H}_{2} \mathrm{~S}$ production and sulfhydration of the NFkB subunit RelA, increasing DNA binding (332). Combined effects of NO on CBS and CTH are probably compensatory regarding $\mathrm{H}_{2} \mathrm{~S}$ production through transsulfuration, in turn, acting on tumor growth.

Importantly, MAT2A expression does not follow the same trend in all tumors, being increased in liver, colon and breast cancers $(364,407)$. According to the Human Protein Atlas, reduced MAT $\alpha 2$ staining is reported in glioma or thyroid tumors and disparities in staining for most transsulfuration proteins exist in different cancer types. The NCI60 human cancer cell panel shows reduced $C B S$ expression as compared to HepG2 hepatoma cells (419) and PDRG1 overexpression occurs in several tumors coinciding with downregulation of p53 and/or miR-214, as expected from their diminished binding to the PDRG1 promoter and mRNA, respectively $(154,291,390)$. This last evidence suggests that in tumors with increased PDRG1 and MAT $\alpha 2$ levels modulation of AdoMet synthesis and DNA methylation can involve their interaction. In this line, aspects such as whether this interaction is restricted to a specific subcellular compartment and the existence of putative MAT $\alpha 2$ localization changes remain unexplored.

\subsection{Ethanol abuse}

In 1947, it was reported that patients with alcohol liver cirrhosis exhibit a delayed clearance of plasma methionine after an overload of this amino acid (175). It is also long-known that the disease often concurs with deficiencies in B-vitamins required in transsulfuration. Later on, impaired transsulfuration, reduced hepatic MAT and PEMT activities and low AdoMet concentrations were described in these patients (84,141). The MAT I/III activity ratio was decreased by enhanced MAT III levels, concomitant with MAT I loss (33). These results were confirmed in animal models of 
Pajares and Pérez-Sala

ethanol and $\mathrm{CCl}_{4}$ intoxication $(64,127)$, where parallel depletion of glutathione and alteration of the GSH/GSSG ratio were detected. Moreover, inhibition of GSH synthesis with buthionine sulfoximine (BSO), an inhibitor of GCL, also decreased MAT activity and AdoMet concentrations, effects that were prevented by glutathione monoethyl ester (EGSH) (65). Additionally, GSH transport into the mitochondria was reduced by the decreased fluidity of the inner mitochondrial membrane induced by ethanol, effects prevented by AdoMet (63). Ethanol fed micropigs showed increased plasma Hcy and hepatic AdoHcy levels, centrilobular microsomal CYP2E1 and acetaldehyde and malondialdehyde protein adducts (127), and ethanol intoxicated rats presented DNA hypomethylation, increased DNA strand breaks and $c-M Y C$ expression (217). The oxidative stress and proinflammatory response elicited by ethanol are linked, and reflected in the expression changes induced by $\mathrm{NF \kappa B}$ activation. Expression in human cirrhosis resembles changes in $\mathrm{HCC}$, the MAT1A promoter is hypermethylated, and hence, expression reduced together with that of GNMT, $C B S$ and remethylating genes (12) (Fig. 14). Additionally, lncRNA HEIH levels increase in cirrhosis and HCC, enhancing its binding to EZH2 and recruitment to the target genes (402).

\subsection{Acute liver injury}

Acetaminophen (APAP) intoxication is the leading cause of acute liver injury in the USA and UK. Its metabolization involves the production of NAPQI, an intermediate able to modify and inactivate proteins, and requires GSH (301). Therefore, administration of the glutathione precursor $\mathrm{N}$-acetylcysteine (NAC) is the treatment of choice to maintain adequate GSH concentrations. As expected from the link between GSH synthesis and transsulfuration, APAP intoxication in rodents reduces hepatic Gamt and Bhmt expression and increases that of Mat $2 a$ with the consequent reduction in AdoMet levels (330). Effects in other transsulfuration related genes include transient 
Pajares and Pérez-Sala

increases in Gnmt, Ahcy and Gclc transcripts. Cytosolic MAT 11 levels remain unaffected by APAP in rat liver, but homotetrameric association is preferred and MAT activity decreases (71). The net result is a reduction in AdoMet content, together with a stronger decrease in AdoHcy and GSH concentrations, leading to an increased methylation index and reduced $\mathrm{GSH} / \mathrm{GSSG}$ ratio, respectively. Additionally, the decreased GSH/GSSG ratio induced by APAP correlates with nuclear accumulation of MAT $\alpha 1$. NAC supplementation to H35 transfected cells prevented changes in the GSH/GSSG ratio and MAT 1 localization (71,293). Nuclear MAT activity remains unaffected by APAP, despite the increase in MAT I content and H3K27me3 levels. The underlying cause for the lack of activity changes remains unknown, but may rely on the putative modification of MAT $\alpha 1$ by NAPQI. Furthermore, the importance of transsulfuration and associated reactions in acute liver injury is also supported by the better response exhibited by mouse strains with high Bhmt2 expression against APAP intoxication (212). This effect may depend on the use of S-methylmethionine as methyl donor, doubling the yield of methionine recovered by Hcy remethylation.

More data linking acute liver injury and transsulfuration have been obtained in models of D-galactosamine (Gal) intoxication, that cause depletion of uridine nucleotides and the subsequent impairment of RNA and protein synthesis, and GSH depletion by BSO. Gal-intoxication induces the hepatic Matla/Mat2a switch and reduction of Gnmt and Bhmt expression, while increasing Mat2b, Ahcy, Mtr, Gclc, Gclm and Pdrgl transcript levels (71,291) (Fig. 14). Generally, changes in mRNA levels correlate with abnormalities in their half-lives. Expression and cytoplasmic protein levels follow the same trend, and homotetrameric association is preserved in GNMT, AHCY and BHMT, whereas MAT $\alpha 1$ is mainly homodimeric $(33,71)$. Subsequently, the corresponding activities change, according to the protein levels and the association 
Pajares and Pérez-Sala

state, and the hepatic metabolite concentrations are altered. Hence, AdoMet concentration decreases and Hcy, methionine and GSSG levels increase, reducing the methylation index and GSH/GSSG ratio (71).

Gal- and BSO intoxications reduce MAT $\alpha 1$ and BHMT cytoplasmic content, while inducing their nuclear accumulation in H35 cells $(71,293)$. Gal induces a similar nucleocytoplasmic redistribution of GNMT, whereas AHCY and PDRG1 only increase their nuclear levels $(71,291)$. Both agents diminish the GSH/GSSG ratio and prevention of this change by NAC or EGSH administration precludes anomalous nucleocytoplasmic distribution of MAT $\alpha 1$ and BHMT due to Gal or BSO, respectively $(71,293)$. In contrast to APAP intoxication, nuclear MAT $\alpha 1$ accumulation in Galintoxicated livers rises MAT I content and MAT activity (71). Moreover, Gal-treated hepatoma H35 cells also show increased H3K27me3 levels correlating with nuclear MAT $\alpha 1$ accumulation and NAC administration prevents alterations in this repression mark. Lack of significant effects in DNA methylation may rely on the parallel Galinduced nuclear accumulation of PDRG1 (291). Interaction between PDRG1 and MATs reduces their activity, putatively decreasing nuclear AdoMet concentrations, despite MAT I increase. In this context, nuclear methyltransferases with higher affinities for the methyl donor (e.g. EZH2) would be less affected by its limited availability. Altogether, these data suggest that redox changes induced in acute liver injury play an important role in subcellular distribution of transsulfuration enzymes and their nucleocytoplasmic activities. Therefore, it may be worth to increase research in this field towards new drugs that avoid/modulate these effects to expand the available therapeutic options.

\subsection{Wilson disease}

Wilson disease is characterized by hepatic copper accumulation and impairment of its transport into the bile, although some patients present neurological deficiencies. 
Pajares and Pérez-Sala

These symptoms are a consequence of various mutations in the $A T P 7 B$ copper transporter gene. D-penicillamine is widely used for the treatment of Wilson disease and, in 1975, its coadministration with AdoMet showed improvement of liver function (104).

Oxidative stress, as well as alterations in methionine metabolism have been identified early in life in two models of this disease, the Long Evans Cinnamon rat (LEC, with a $900 \mathrm{bp}$ deletion in Atp $7 b$ ) and the toxic milk mouse (tx-j, carrying the G712D mutation). Livers of 9-weeks old LEC rats already present the Mat1a/Mat2a expression switch, decreased Mtr, Ahcy and, especially, Mat2b transcript levels (72) (Fig. 14). In cells, BSO prevented downregulation of Mat $2 b$ by copper, whereas its chelator neocuproine was uneffective and increased cell death, as expected from the elevated production of the toxic neocuproine- $\mathrm{Cu}(\mathrm{I})$ complex. Once more, hepatic cytoplasmic protein levels in LEC rats matched expression, leading to decreased MAT activity and GSH/GSSG ratios, alongside increased MAT I and MAT II levels (72). Enhanced hepatic methionine concentrations were noticed, probably due to copper activation of MTR, whereas AHCY inhibition cannot explain the low AdoHcy levels found, which result in an increased methylation index. Furthermore, in vitro experiments proved that copper alters the folding/association of MAT $\alpha 1$ and MAT $\alpha 2$ and inhibits the isoenzymes (72). This effect may be the basis for the reduced hepatic AdoMet levels and MAT activity observed in older LEC rats (339), mimicking cirrhosis and HCC.

Evolution of hepatic expression changes in transsulfuration and related reactions with age have been studied using tx-j mice (197). This work revealed downregulation of Ahcy from fetal stages until adulthood, together with biphasic expression trends for other genes. Namely, fetal downregulation of Dnmt genes, Mat2a and Mtr and their 
Pajares and Pérez-Sala

upregulation from 12-20-weeks of age onward occurs, whereas Matla expression boosts in mice 12-weeks old. Therefore, AHCY, the methylation index and global DNA methylation are reduced. Additionally, decreased expression of genes involved in fatty acid oxidation (Ppar $\alpha$ and Cpt1A), lipid synthesis (Srebplc) and ER stress (Grp78) are found, together with the corresponding changes in SREBP1c and PPAR $\alpha$ proteins. In tx-j mice, administration of D-penicillamine or betaine reduces copper levels and normalizes DNA methylation (246). However, these agents exert additional effects, Dpenicillamine increases AHCY activity, whereas betaine reduces Ahcy expression, increases AdoMet levels, the methylation index and Dnmt $3 b$ expression. Choline supplementation of the maternal tx-j mice diet induces global DNA hypermethylation in the fetus, while preventing downregulation of Mat2a, Dnmt1, Dnmt3a, Dnmt3b, Ahcy, Mtr, Srebplc, Cpt1A and Ppara expression (245). In general, DNA hypomethylation correlates with upregulation of Dnmt genes, whereas AdoHcy levels and the methylation index associate with changes in copper levels (197). Altogether, Wilson disease models show impairment of transsulfuration with alterations mimicking mainly those described for HCC and cirrhosis, but also with additional defects e.g. in folding.

\subsection{Hearing loss}

According to the 2013 report of the World Health Organization, there are 360 million people worldwide with moderate to profound hearing loss and the problem affects especially the aged population. Surprisingly, 50\% of the factors involved (e.g. noise, ototoxic drugs) can be prevented, and association of hearing loss with deficits in micronutrient ingestion exists. Epidemiological studies also noticed a relationship between deafness and increased plasma Hcy levels, but $\sim 10-20 \%$ of the human population shows such an increase due to several factors (29). Decreased serum folate levels, correlating with low vitamin $\mathrm{B}_{12}$ or HHcy, are also detected in age-related and 
Pajares and Pérez-Sala

sudden sensorineural hearing loss $(35,195)$. Moreover, these studies provide evidences of the association between inner ear atherosclerosis and deafness (157). This connection with Hcy and folate metabolisms prompted the design of intervention studies that improved age-related hearing loss and reduced systemic Hcy levels (87).

Data obtained from animal models involve Hcy and folate metabolism in hearing loss. $\mathrm{Cbs}^{+/-}$mice present high Hcy levels in the stria vascularis, the structure involved in the metabolic control of the inner ear, and the spiral ligament (190). Moreover, these mice exhibit increased hearing thresholds, oxidative stress and reduced density of the microvasculature, abnormal features that are prevented by folate administration. Reduced Bhmt and increased Ahcy expression are found in the stria vascularis of $C x 30^{-/-}$mice (61), which have impaired connections among cilliary cells in the organ of Corti that are essential for hearing. Nevertheless, the first study devoted to cochlear Hcy metabolism was performed in mouse quite recently (238). Genes of transsulfuration and related reactions show the expected expression pattern for a nonhepatic organ. Namely, high levels of Mat2a, Mat2b, Ahcy and Adk transcripts, intermediate levels for Mtr expression and low for Mat1a, Ada, Cbs and Bhmt2. As an exception, similar Mtr and Bhmt transcript levels are detected. Some proteins, BHMT and AHCY, present anomalous mobilities in SDS-PAGE gels that are compatible with the existence of PTMs or specific cochlear splicing forms that remain unknown.

The impact of the nutritional deficiency of folic acid was analyzed using two mouse models, C57BL/6J mice that are prone to hearing loss and $\mathrm{CBA}$ mice with delayed onset of the disability. In both cases, folic acid deficiency accelerates the onset of hearing loss $(238,239)$, but CBA mice require a long-term exposure to the diet that also leads to anemia, therefore suggesting that the impact of the diet depends on the genetic background (Fig. 15). Nevertheless, the folate-deficient diet causes cochlear 
Pajares and Pérez-Sala

expression and protein changes in $\mathrm{C} 57 \mathrm{BL} / 6 \mathrm{~J}$ mice intended to decrease Hcy production (Ahcy and AHCY), moderate its remethylation (reduced Bhmt, Mtr, and BHMT, but increased MTR) and catabolism (decreased CBS), in an attempt to diminish protein Nhomocysteinylation (238). However, changes in Hcy catabolism may have a dark side by decreasing $\mathrm{H}_{2} \mathrm{~S}$ production by $\mathrm{CBS}$ and subsequently contributing to microvasculature alterations. Several cochlear parameters indicate oxidative stress and an attempt for its control: i) p22phox and MnSOD levels increase; ii) Cat, Gpx4 and GsS expression decreases; iii) GSH and GSSG concentrations rise maintaining the GSH/GSSG ratio; and iv) 3-nitrotyrosine levels increase in the stria vascularis and the cochlear ganglion.

Other epidemiological studies showed the putative protection offered by $\omega 3$ PUFA against hearing loss $(85,119)$. This possibility was initially explored in shortterm supplementation studies of animal models, rendering contradictory results. However, a long-term supplementation study of C57BL/6J mice shows modest protection against hearing loss, together with attenuated cochlear inflammation and oxidative stress (240). Regarding transsulfuration and associated reactions, decreased expression of Ahcy, Cbs, Cth and Adk was detected with age, as well as for Mtr and Bhmt remethylation genes, whereas $A d a$ was upregulated. Protein levels remained mostly unmodified with age, except for a CBS increase that was prevented by $\omega 3$ supplementation, while boosting BHMT content. This effect could rely on choline recycling as a consequence of anti-inflammatory mediator production from PCincorporated PUFAs. This possibility is supported by the increased hepatic Pemt and Gnmt expression detected in $\omega 3$-supplemented animals, although their cochlear expression remains unknown. Conversely, the increased CBS detected with age could reflect an enhanced $\mathrm{H}_{2} \mathrm{~S}$ need to regulate the synthesis of anti- and pro-oxidant enzymes 
Pajares and Pérez-Sala

and inflammatory mediators (161). In fact, several synthetic drugs that provide this gasotransmitter have been used to treat deafness with diverse outcomes. Protection against noise-induced hearing loss is provided by nicotinamide riboside (31), a NAD ${ }^{+}$ precursor. This treatment prevents noise effects in hearing and neurite degeneration in the spiral ganglion and in mitochondrial SIRT3 that requires this cofactor. SIRT3 malfunction induces ROS and reduction of GSH content (342). However, effects of this treatment on AHCY that also uses $\mathrm{NAD}^{+}$were not analyzed, despite the potential improvement of nicotinamide riboside administration in cochlear Hcy metabolism. 
Pajares and Pérez-Sala

\section{DETOXIFICATION MECHANISMS LINKED TO TRANSSULFURATION}

Detoxification of xenobiotics and endogenous electrophilic compounds that are generated in excess under pathophysiological conditions involves a series of processes some of which require metabolites derived from sulfur amino acid metabolism, such as AdoMet or GSH $(26,249,267)$. Additionally, these reactions produce the oxidized forms of different cofactors (e.g. $\mathrm{NADP}^{+}$) that regulate transsulfuration and related reactions $(115,250)$. Drug metabolism is essential for processing towards inactive metabolites and for the transformation of prodrugs into their active metabolic forms. Enzymes involved in these processes catalyze conversion of the original compound into a more polar metabolite (phase I) or conjugation reactions for the transformation of endogenous compounds and xenobiotics into more hydrophilic products (phase II) for excretion or inactivation of pharmacological drugs. These enzymes present ubiquitous expression, but their highest levels exist in liver. In this review, we have focused in a few of such processes that exemplify the connections with transsulfuration.

\subsection{Aldo-ketoreductases}

The aldo-ketoreductase (AKR) family of enzymes plays an important role in the reduction of carbonyl-containing compounds, which requires adequate levels of the NADPH cofactor (250). This family comprises more than 150 members with high sequence conservation and a similar monomeric structure. AKR1B1 and AKR1B10 are probably the best studied due to their involvement in the development of diabetic complications and/or cancer. These roles are related to their ability to metabolize aldehyde-substrates such as glucose, endogenous reactive carbonyls such as HNE and acrolein that are cytotoxic, and several anti-cancer drugs $(79,179)$. Under physiological conditions, these enzymes play a constitutive and/or inducible cell-protective role. Thus, 
Pajares and Pérez-Sala

in certain cell lines, oxidants or electrophilic compounds induce $A K R$ expression through factors such as $\operatorname{Nrf} 2(166,224)$.

AKR1B1 catalyzes removal of $\sim 30 \%$ of glucose excess in diabetes. Hence, its pathological role derives from an increased flux through the polyol pathway resulting in elevated NADPH consumption and redox imbalance (295,345). Additionally, the sorbitol excess generated can induce osmotic stress that together with AKR1B1 role in inflammation can further contribute to diabetic complications. In fact, certain AKR1B1 polymorphisms have been associated with complications such as diabetic cataract, retinopathy, nephropathy, vasculopathy, etc. (73).

Several AKRs have been associated with cancer and, particularly, AKR1B1 and AKR1B10 are overexpressed in lung, liver and breast tumors (192). The proposed mechanisms include the AKR1B10 abilities to: detoxify endogenous carbonyls increasing survival (389); metabolize exogenous carbonyls leading to chemoresistance (132); associate with acyl-coenzyme A carboxylase promoting fatty acid synthesis and, subsequently, tumor proliferation (225); and competence to convert retinaldehyde into retinol hampering activation of retinoic acid receptor and cell differentiation (103). AKR1B10 also metabolizes PAH to quinones with higher carcinogenic potential (303), thus interfering with PAH binding to the transsulfuration enzyme GNMT. Nevertheless, the role of AKRs in tumor promotion appears more complex, since AKR1B10 expression increases in well-and moderately-differentiated liver tumors, but decreases in advanced-poorly differentiated tumors (135). In HCC and preneoplasic liver, AKR1B10 expression is increased, whereas siRNA-mediated knockdown inhibits HCC cell proliferation $(329,369)$. However, high AKR1B10 levels also predict favorable prognosis in HCC, after curative hepatectomy (124). Altogether, it remains unclear 
Pajares and Pérez-Sala

whether the enzyme exerts a defensive role for the host in early tumor stages that is lost in more advanced steps.

Importantly, AKRs can participate in prostaglandin synthesis and are redox regulated though various oxidative and electrophilic modifications on cysteines in their C-terminal segment, close to the active site. These modifications either increase (e.g. nitrosylation and acrolein) or decrease enzyme activity (e.g. cyclopentenone prostaglandins (cyPG), HNE, glutathionylation) (346). Moreover, the cofactor NADPH also protects AKRs from inactivation by 4-oxonon-2-enal $(82,230)$, whereas GSH availability and glutathionylation impact AKR activity allowing recovery from cyPGmodified or nitrosylated states, respectively, protecting the enzyme against irreversible inactivation $(14,80)$. These last effects provide additional connections between transsulfuration, GSH synthesis and detoxification.

\subsection{Glutathione S-transferases}

Glutathione S-transferases (GSTs) are multifunctional enzymes that play a key role in the maintenance of the redox status and cellular detoxification. Conjugation of multiple polar compounds with GSH is their classical function, but they also form channels in intracellular membranes, reduce the availability of a variety of compounds by covalent and non-covalent binding (267), catalyze protein glutathionylation, thus participating in the defense against oxidative damage (362), and perform reactions involved in prostaglandin and steroid hormone synthesis (28). This superfamily comprises cytosolic, mitochondrial and microsomal members, whose structural and functional features have been reviewed elsewhere (267). Cytosolic GSTs can also move to the nucleus and differences in their nucleocytoplasmic activities correlate with protein-adduct production in either compartment (350). 
Pajares and Pérez-Sala

Increased GST activity occurs in tumors, correlating with chemoresistance. In fact, GST has been considered a marker of hepatic carcinogenesis (321). GST induction can act as defense mechanism against chemicals, but once overexpressed it may provide an advantage for transformed cells that become able to survive the toxic insult. However, in tumor cells the GSH required for GST activity may not be sustained due to impaired transsulfuration, making them dependent on a larger import of cysteine. GSTP1-1 is one of the most exhaustively studied members of this family. Nrf2 upregulates GSTP1 expression in HCC through its binding to ARE elements in the promoter (196). Upregulation is also detected at initial stages of chemical hepatic carcinogenesis, as expected from the ability of these agents to change its transcription $(133,169)$. However, modification of GSTP1-1 in response to several electrophilic compounds and drugs (e.g. HNE, cyPG, oxidants) modulates its oligomerization and activity (322). Among others, agents inducing oxidative stress or cyPG inhibit GST, eliciting reversible dimerization or irreversible tetramerization through cysteine crosslinking, respectively (323). Many of these compounds are also GST substrates, thus a context-dependent balance between their detoxification and enzyme inhibition is established. In fact, GSH or non-hydrolyzable GSH analogs protect GST against these modifications and oligomerization (106,322). Protein-protein interactions involving monomeric GST can be impaired by oligomerization, subsequently leading to activation of stress cascades by the release of its putative interaction partners. In fact, this mechanism supports sustained JNK activation directing to apoptosis $(1,20)$.

GST provides protection against injury by various agents, e.g. its induction protects against acute liver failure (113). Surprisingly, Gstp $p^{-/-}$mice exhibit increased resistance against APAP-induced liver injury, without induction of other GST family members, but with compensatory rises in enzymes maintaining redox status (e.g. 
Pajares and Pérez-Sala

antioxidant protein 2, thioredoxin peroxidases) (176). This reduced toxicity also correlates with increased global protein glutathionylation, including modification of GSH biosynthesis enzymes, and could represent an adaptive mechanism providing protection in the absence of GSTP (243). Altogether, the protective mechanisms involving GST are constituted by a complex network of regulatory proteins and compensatory systems to which e.g. the different BHMT2-dependent susceptibilities to APAP may contribute. Moreover, although human and mouse GSTPs are highly homologous, critical residues for modification by oxidants and electrophiles (C101) are not preserved in the mouse protein, and hence the response to these agents may differ between species.

\subsection{Thiopurine methyltransferase}

Thiopurines such as 6-mercaptopurine (6MP) and its precursor azathioprine are the treatments of choice for inflammatory bowel disease, Crohn's disease and ulcerative colitis, but $6 \mathrm{MP}$ is also prescribed in childhood acute lymphoblastic leukemia (ALL) and azathioprine as immunosuppresor $(11,331)$. Azathioprine is converted mainly by GST into 6MP, a process dependent on the activity and polymorphisms of this protein family (229). Three competing routes further metabolize 6MP using thiopurine methyltransferase (TPMT), hypoxanthine phosphoribosyltransferase (HPRT) or xanthine oxidase. Thioxanthine, an intermediate of the xanthine oxidase pathway, inhibits TPMT (26), while enhancing HPRT activity (331). 6MP combined with methotrexate is administrated as maintenance treatment for ALL, but methotrexate, as folate analog, inhibits purine and pyrimidine synthesis and dihydrofolate reductase, impairing folate recycling and remethylation (49).

TPMT is a cytoplasmic phase II enzyme, whose physiological substrate remains unknown. Its expression is almost ubiquitous, although hepatic and renal levels are the 
Pajares and Pérez-Sala

highest. TPMT polymorphisms reducing activity are frequent and, in these cases, toxic effects of thiopurine treatments can increase mortality (229), therefore the importance of pharmacogenetic TPMT tests in chemotherapy. Azathioprine and 6MP are hepatotoxic drugs, but this effect is also observed with 6-methylmercaptopurine the product of TPMT (83). Although the exact contribution of TPMT to AdoMet consumption is not well established, these therapies will increase AdoMet needs in tumor cells that have reduced MAT activity, thus favoring 6MP metabolization through competing pathways. The 6-thioguanine nucleotides generated can substitute adenine and guanine in the DNA, inhibiting replication and contributing to DNA damage and apoptosis (302). AdoMet addition to MOLT lymphoblasts showed TPMT stabilization and prevention of 6MP cytoxicity (249). However, the decreased DNMT activity and global hypomethylation observed seem to depend on a metabolite not produced by TPMT (139). Altogether, the ATP required for AdoMet synthesis to meet TPMT and DNMT needs depends on de novo purine synthesis that is inhibited by methylated products of 6-thioinosine monophoshate also generated by TPMT (249), thus closing the loop and favoring cytotoxicity. 
Pajares and Pérez-Sala

\section{HUMAN MUTATIONS IN TRANSSULFURATION}

An increasing number of patients with homocystinuria, hypermethioninemia, mild liver dysfunction and/or neurological defects due to genetic deficiencies in enzymes of transsulfuration and associated reactions have been identified along the years. The variety of symptoms and deficiencies associated with transsulfuration impairment may rely on the diversity of AdoMet-dependent processes, but may also obscure defects concerning other metabolites with a more restricted utilization. The impact of the mutations found to date can be inferred from the structural information that is slowly becoming available. Nevertheless, since oligomerization is in many cases essential to achieve the biological function, knowledge about the folding and association events is also crucial to interpret the role of certain mutations with an apparently minor impact.

\subsection{Human mutations identified in transsulfuration genes}

According to the NCBI-SNP database on February 2017, missense mutations, frameshifts and splicing forms have been identified in most transsulfuration genes (Table 1). Homozygous and compound heterozygous mutations are commonly found, but some heterozygous mutations have been also identified. In general, these mutations are inherited in an autosomal recessive trait, but some exceptions exist. Homocystinuria (MIM 236200), discovered in 1962 in patients with mental retardation, is the most studied disorder (44). It is characterized by increased urinary excretion of homocistine and methionine and the associated symptoms include mental retardation, skeletal abnormalities and myopia. Newborn screenings are carried out worldwide, allowing detection of an increasing number of $C B S$ mutations. The most common mutation, I278T, associates with the risk of spina bifida and leads to reduced protein stability and minimal activity (56). Prevalence of CBS deficiency varies notably among countries, 
Pajares and Pérez-Sala

but is estimated between 1:58000 and 1:1000000. Response to vitamin $\mathrm{B}_{6}$ depends on the mutation, for example patients carrying the $\mathrm{I} 278 \mathrm{M}$ or $\mathrm{A} 114 \mathrm{~V}$ mutations are pyridoxine responsive, whereas those with the G307S mutation are non-responsive.

Cystathionuria (MIM 219500) due to mutations in the $C T H$ gene was described in a mental institution in 1959 (128). The estimated prevalence is 1:14000. These patients show increased plasma concentrations and urinary excretion of cystathionine. Again, different outcomes to vitamin $\mathrm{B}_{6}$ treatment have been described. The T67I mutation of ancient origin is the most common and responsible for the loss of CTH activity (91). Recombinant T67I protein shows reduced affinity for the $\mathrm{B}_{6}$ cofactor, as expected from its localization in the same chain than R62 that is involved in vitamin binding (186). R62H and Q240E mutations also affect CTH activity, the former by altering cofactor binding and increasing conformational flexibility near the active site and the latter by changing kinetic parameters (186). Additional mutations include deletion of residues G57-Q196, consequently eliminating a large portion of the catalytic site and putatively modifying tetramer association.

The number of cases of isolated persistent hypermethioninemia due to MATIA (MIM 250850), AHCY (MIM 613752) and GNMT (MIM 606664) mutations is more limited, and hence their worldwide prevalence remains unknown. MATIA deficiency presents with elevated plasma methionine levels and is usually asymptomatic, although some cases with neurological abnormalities have been described. Demyelination was initially observed in patients with indels leading to truncated MAT $\alpha 1$ forms $\sim 350$ residues long, but later detected in individuals carrying shorter protein forms (185X) or single mutations (R292C/R356L) (259,262,354). AdoMet treatment improved myelination in some patients $(262,354)$. $\mathrm{R} 264 \mathrm{H}$ is the most common MAT1A mutation and the only autosomal dominant (51). This mutation reduces enzyme activity, an effect 
Pajares and Pérez-Sala

ascribed to the involvement of the wild type arginine in a salt bridge essential for dimerization.

Patients carrying GNMT mutations exhibit high plasma methionine and AdoMet levels, together with increased serum transaminases and mild hepatomegaly. The few mutations identified to date are point mutations that reduce activity. L49P disturbs the second $\alpha$-helix, H176N decreases stability and N140S nearly abolishes catalysis $(219,222,223)$.

AHCY deficient patients present elevated plasma AdoHcy, AdoMet and methionine levels, their symptoms including mental and motor retardation, facial dismorphy and myocardiopathy (17). The few point mutations identified to date affect the catalytic domain. R49C and Y143C include new cysteine residues putatively contributing to alter the cofactor's oxidative state, while others like D86G suppress negative charges in this domain $(17,19,383)$. Some mutations also increase thermosensitivity or contribute to form inactive aggregates. Interestingly, a translocation involving TAX1BP1 and $A H C Y$ genes has been described in breast cancer HCC1806 cells (142), although it remains unclear whether the fused gene is translated. Such anomaly may interrupt several processes, including TAX1BP1 interaction with the $\mathrm{NF \kappa B}$ signaling pathway and its coactivation of nuclear receptors, as well as AdoHcy hydrolysis with the corresponding reduction of the methylation index and methyltransferase inhibition.

Cerebral creatine deficiency syndrome 2 (MIM 612736) due to mutations in the GAMT gene leads to developmental delay, mental retardation and muscular hypotonia, among other symptoms. Patients have low plasma and reduced excretion of creatine. Point mutations W20S and C169Y reduce GAMT activity (37), whereas T136M alters the protein core (7). Indels are also detected in the GAMT gene causing changes in the 
Pajares and Pérez-Sala

sequence and early stop codons $(7,149)$. Some truncated proteins lack D134, an essential amino acid for AdoMet binding, and consequently their activity is abolished.

Data about the impact of mutations in the remaining transsulfuration genes are scarce. Nevertheless, the V175M mutation in PEMT causes activity loss and associates e.g. with the susceptibility to non-alcoholic fatty liver disease (343), whereas the Cterminal E344A and $\mathrm{R} 356 \mathrm{H}$ mutations in MAT $\alpha 2$ seem to predispose to thoracic aortic disease (122). These MAT $\alpha 2$ mutations alter association and/or activity due to their respective locations at the end of an $\alpha$-helix required for electrostatic interactions with the other monomer or MAT $\beta$ (E344A) and near the AdoMet binding site $(\mathrm{R} 356 \mathrm{H})$. Regarding DNMTs and EZH2, mutations have been reported as stated in Table 1, but no specific disease has been related to changes limited to these genes.

\subsection{Impact of mutations in association and subcellular localization}

Most transsulfuration proteins are homo or hetero-oligomers, and association is a requirement to attain activity. Subunit assembly requires not only preservation of the overall structure, but also of the charge or hydrophobic characteristics of the interacting interfaces. Some of these features may be altered in the variant proteins obtained by alternative splicing that are detected in pathologies that concur with oxidative stress. In fact, those reported to date result in: i) change (MAT $\beta$, PEMT) or elimination (AHCY) of part of the N-terminal sequence; ii) deletion or alteration of other parts of the sequence (MAT $\beta$, PEMT, GAMT); iii) insertions (CBS); and iv) the combination of these factors (e.g. MAT $\beta-V 2 a)$. Additionally, mutations leading to early stop codons or frameshifts may have a similar impact.

Examples of special interest to explain these effects are MAT $\alpha 1$ truncated forms. The anomalous association of MAT $\alpha 1350 \mathrm{X}$ or $351 \mathrm{X}$ with wild type MAT $\alpha 2$ subunits in an inactive oligomer was initially proposed to explain demyelination in 
Pajares and Pérez-Sala

these patients (130). Later on, when shorter forms (185X) were found in patients with these symptoms and the MAT I/III structure became available (116), it was evident that association requires three $\beta$-sheets, provided each by a different domain, and that $185 \mathrm{X}$ cannot meet this constraint. In contrast, the $350 \mathrm{X}$ truncation eliminates $\sim 50 \%$ of the $\mathrm{C}$ terminal domain preserving the sequence for the $\beta$-sheet, whereas in $351 \mathrm{X}$ the remaining sequence of this domain changes (228). Therefore, only the $350 \mathrm{X}$ subunit may achieve an intermediate folding state resembling that allowing association with MAT 2 2. Provided this is the case, two scenarios can take place: i) monomer association in an inverted fashion to render a MAT $\alpha 1 / \mathrm{MAT} \alpha 2$ heterodimer with a single active site completed; and ii) the $350 \mathrm{X}$ intermediates could trap MAT $\alpha 2$ intermediates and preclude the last folding steps. A putative hetero-oligomeric association of $351 \mathrm{X}$ and MAT 22 intermediates seems even more complicated, as only two "wild type" domains could be involved, in turn, increasing the instability of the association or generating an unproductive intermediate due to the alternative C-terminal sequence.

Subcellular localization can be also modified by mutations. Cytoplasmic retention and nuclear localization of MAT $\alpha 1$ involves C-terminal basic residues, their elimination putatively altering subcellular distribution. In fact, nuclear accumulation and $70 \%$ reduction of MAT activity is detected in the rat $\mathrm{N} 351 \mathrm{X}$ protein $(311) . \mathrm{T} 87 \mathrm{~N}$ and D234N mutations in CBS also affect localization (46), despite their situation out of the protein's C-terminal end required for the interaction with the sumoylation machinery (162). Effects were stronger for the T87N mutant that abolishes activity and tends to aggregate (46). This highly conserved residue locates in a loop within the Nterminal domain, its side chain putatively stabilizing the hydrophobic dimer interface, as well as establishing interactions with additional residues. Substitution of threonine by 
Pajares and Pérez-Sala

asparagine may disrupt the array of interactions established by the former, in turn, destabilizing the oligomer. 
Pajares and Pérez-Sala

\section{INTEGRATED VIEW OF TRANSSULFURATION}

The conversion of methionine into cysteine through transsulfuration involves the synthesis of key metabolites that allow the interplay of this pathway with many others. AdoMet-dependent methylations are key for this exchange transferring the methyl group to multiple substrates for the synthesis of a variety of compounds or the regulation of processes through protein, RNA and DNA modification (218). Here, we have highlighted AdoMet connections including the synthesis of sarcosine (219), creatine (158) and PC (273), drug detoxification (249) and epigenetic modifications $(285,400)$, but we cannot forget that this network expands far beyond. Besides one carbon metabolism, Hcy connections are evolving to regulatory actions on ER stress and epigenetics, mainly involving protein homocysteinylation (152). Its conversion into cysteine supports GSH synthesis $(256,382)$, key for drug detoxification, but also provides $\mathrm{H}_{2} \mathrm{~S}$ for additional regulatory mechanisms (393). Detection of transsulfuration enzymes in new subcellular localizations $(71,162,187,304,311)$, together with their involvement in unexpected functions (e.g. GNMT as PAH receptor) are changing the classical view.

Nowadays we can no longer speak about transsulfuration as a cytoplasmic process, excepting some transmethylation events, but rather about a pathway with cytoplasmic and nuclear branches as shown in liver (Fig. 16). The hepatic composition of each branch includes as core components MATs, AHCY and CBS $(71,162,304,311)$, whereas differences may rely on the associated methyltransferases. Nevertheless, some are found in both compartments, namely GNMT and GAMT $(158,187)$, as well as the remethylating enzyme BHMT (293). In normal liver, the nuclear content of these enzymes is rather small, but active oligomers exist (e.g. MAT I) together with additional protein states putatively responsible of their moonlighting activities. This is 
Pajares and Pérez-Sala

the case of GNMT dimers, acting as PAH receptors for the activation of XREs (349), and could be that of MAT $\alpha 1$ monomers as activators of the MAT1A E-box (408), although this last option remains unexplored.

Nuclear accumulation of transsulfuration proteins is observed in APAP(MAT $\alpha 1)$ and Gal-induced (MAT $\alpha 1, \mathrm{AHCY}$, GNMT, BHMT) acute liver injury, concomitantly with their cytoplasmic decrease (71,293) (Fig. 16). This altered nucleocytoplasmic distribution correlates with a lower GSH/GSSG ratio and, in some instances, can be prevented with glutathione precursors, mainly NAC. Reductions in the GSH/GSSG ratio and the cytoplasmic content of transsulfuration enzymes are common to other hepatic pathologies, suggesting that similar changes in subcellular distribution could take place in those diseases. In fact, nuclear MAT $\alpha 1$ accumulation has been also reported in HCC (405). Nuclear protein accumulation correlates with increases in processes requiring enzyme activity (e.g. MAT 11 and H3K27me3 or AHCY and cap methylation), indicating that transsulfuration occurs in this compartment. Moreover, it seems plausible that protein accumulation results from an attempt to increase the flux through the nuclear pathway in order to respond to different types of insults, but whether this effect is aided by the nuclear import/export of metabolites such as AdoMet or AdoHcy remains unknown.

Most of the data presented in this review suggest a preeminent role for redox regulation in expression of transsulfuration genes that can be exerted through E-boxes, AP-1 sites or AREs on several gene promoters, which may bind redox regulated transcription factors (e.g NFאB). In normal liver, binding of MAT $\alpha 1 / \mathrm{Mnt}$ or PHB1, an ARE coactivator (96), to the MAT1A E-box regulate its high expression levels, whereas USF-1 binding on the $C B S$ E-box is responsible for basal activity $(108,408)$. Changes in the factors bound to the E-box and/or an interplay with additional elements alters 
Pajares and Pérez-Sala

expression of the corresponding gene (e.g. cMyc/MafG/cMaf binding reduces MAT1A expression). Binding of NFאB, AP-1 or other factors to AP-1 sites increase expression of MAT2A, MAT2B, EZH2, GSTP1-1 in cancer $(98,169,404,409)$ and of CTH upon lipopolysaccharide administration (392). The presence of AREs in GSTP1-1 and AKR promoters allows their regulation through AP-1 or Nrf2 binding as part of the antioxidant response $(133,166)$. These effects are also complemented by alterations in the methylation status of the promoters and their associated proteins due to redox effects on methyltransferases/demethylases or the availability of AdoMet, but also through the interplay of DNA methylation with additional epigenetic modifications dependent on other metabolic pathways (e.g. deacetylation requires $\mathrm{NAD}^{+}$). Thus, the roles of transsulfuration as provider of AdoMet, hydrolyser of AdoHcy and as a "receptor" of epigenetic methylations reciprocally connect this pathway and Epigenetics (Fig. 17). Some of these aspects may also influence post-transcriptional mechanisms, as is the case of miRNA expression that is upregulated in diseases concurring with oxidative stress (e.g. miR-664 in HCC) or the methylation status of HuR $(378,405)$.

Oligomerization, a prerequisite for the function of many transsulfuration enzymes, can be redox regulated as shown for MAT isoenzymes. Moderate decreases in the GSH/GSSG ratio lead to dimeric MAT III accumulation, putatively favoring production of the C35-C61 disulfide bond during folding (235,325), whereas its severe reduction may impair folding and generate monomeric proteins $(234,276)$. In contrast, decreased NADPH/NADP ${ }^{+}$ratios favor MAT II generation by enhancing $\mathrm{NADP}^{+}$ binding to MAT $\beta-\mathrm{V} 1$ and, subsequently its affinity for MAT $\alpha 2$ dimers (115). It remains unclear whether this mechanism favoring MAT II assembly is also feasible for other MAT $\beta$ splicing forms detected in HCC. Altogether, oxidative stress diminishes AdoMet production by supporting the low $\mathrm{V}_{\max }$ isoenzyme MAT II vs. MAT I/III and connects 
Pajares and Pérez-Sala

transsulfuration with the synthesis/levels of redox couples (NADPH/NADP ${ }^{+}$, GSH/GSSG), making it susceptible to their imbalance during detoxification. Again, reciprocal links between transsulfuration and detoxification are established, the former providing AdoMet and cysteine for TPMT activity and GSH synthesis, respectively, while the latter generates oxidized species (i.e. $\mathrm{NADP}^{+}$) that impair transsulfuration.

Protein-protein interactions provide additional regulatory options. Among them, interactions of EZH2 within the PRC2 sustain its methyltransferase activity for the introduction of the $\mathrm{H} 3 \mathrm{~K} 27 \mathrm{me} 3$ repression mark on target genes, whereas interaction of PDRG1 with MAT $\alpha$ subunits reduces AdoMet synthesis (291). This aspect is of special importance in the nucleus, where the PDRG1/MAT $\alpha 1$ interaction occurs, but also during acute liver injury when nuclear accumulation of both proteins arises. Here, again redox regulation may have a role, as suggested by the increased tripartite MAT $\beta / \mathrm{HuR} /$ SIRT1 stabilizing interaction induced by the antioxidant resveratrol (410), which also connects members of transsulfuration with epigenetic processes. Moreover, GNMT and CTH also establish protein-protein interactions that are involved in xenobiotic response (e.g. GNMT/ARNT/HIF-1 $\beta$ ) or in the putative control of autophagy (e.g. CTH/HMGB1) $(349,358)$.

Another level of regulation includes PTMs and their effects on activity or subcellular localization. Here, redox regulation (e.g. MAT $\alpha 1$ nitrosylation or CBS glutathionylation) adds to a large variety of additional mechanisms that connect transsulfuration with acetyl-CoA (e.g. MAT $\alpha 2$ and EZH2 acetylation) or ATP levels (e.g. PEMT, DNMTs and EZH2 phosphorylation) to regulate activity of the different transsulfuration enzymes. In general, oxidative stress reduces initial steps of transsulfuration (e.g. AdoMet synthesis), but enhances the last steps towards cysteine production (e.g. CBS activity). In some instances, an interplay between different PTMs 
Pajares and Pérez-Sala

is established. This is the case of acetylation that interferes with certain phosphorylation events favoring EZH2 stability (388). CBS sumoylation drives the enzyme to the nucleus (162), but no information exists about putative PTMs involved in the nuclear accumulation of transsulfuration enzymes observed in acute liver injury. Collectively, information concerning PTMs (e.g. protein methylations) has been mostly obtained in high-throughput studies, and hence their role remains mostly unknown.

Dietary effects are exerted mainly through the availability of essential substrates or cofactors (e.g. methionine and B-vitamins) for transsulfuration. However, additional aspects include its coordination with other pathways, such as protein synthesis (e.g. EF$1 \alpha$ induction) and autophagy (e.g. PP2A methylation) by methionine excess $(50,355,357)$, or the regulation of expression of transsulfuration genes through nutrient sensing elements (e.g. FXR in $C t h)$ (241). Furthermore, methionine restriction changes global transcriptional and translational programs, increasing for example GSH and xenobiotic metabolism by cytochrome P450 (110) and ATP synthesis by oxidative phosphorylation (426). Thus, the diet further contributes to reciprocal connections between transsulfuration, additional metabolic pathways and expression events. 
Pajares and Pérez-Sala

\section{CONCLUSIONS AND FUTURE DIRECTIONS}

Many diseases, but also nutritional changes and detoxification events, concur with alterations in transsulfuration that extend from gene expression towards enzyme activity, modifying the availability of essential metabolites for the synthesis of cellular components and regulatory mechanisms. The redox status is involved in a variety of such alterations, through differential binding of transcription factors, introduction of PMTs, changes in subcellular localization and putatively by promoting pathological protein-protein interactions. However, most of these aspects need additional examination. This picture is further complicated by the presence of key core proteins of transsulfuration in the nucleus, where they show activity, thus indicating the existence of cytoplasmic and nuclear branches of the pathway. Aspects as whether nuclear transsulfuration is enough to support e.g epigenetic modifications, the actual nuclear metabolite concentrations (e.g. AdoMet) or if the cytoplasm still contributes for this purpose remain unknown. Accumulation of core proteins during oxidative stress points towards an effort to improve the flux through nuclear transsulfuration, but there is insufficient information concerning putative PTMs or protein-protein interactions involved in nucleocytoplasmic shuttling. Moreover, differences in the oligomeric state of some enzymes between subcellular compartments suggest changes in their structures, which may potentially result in moonlighting activities and/or new interaction surfaces that deserve further investigation. To date, these moonlighting functions link transsulfuration with detoxification and control of gene expression, but the set of regulated genes and transsulfuration proteins involved is limited. Furthermore, the impact of human mutations in these aspects is largely unknown, and hence deserves some attention. The neurological symptoms associated with human mutations and the large variety of disease that concur with impairment of transsulfuration also highlights 
Pajares and Pérez-Sala

the need to increase the knowledge on this pathway in non-hepatic tissues. Altogether, transsulfuration presents an interesting interplay between Nutrition, a variety of metabolic pathways, redox regulatory processes, epigenetic and detoxification events that are far from being completely understood and that constitute a continuous source of surprises. 
Pajares and Pérez-Sala

\section{ACKNOWLEDGEMENTS}

This work was support by grants of the Ministerio de Economía $y$ Competitividad (SAF2012-36519 and SAF2015-68590R to DPS) and Instituto de Salud Carlos III (RETICs RIRAAF RD12/0013/0008 and ARADyAL RD16/0006/0021). 
Pajares and Pérez-Sala

\section{ABBREVIATIONS}

ADA, adenosine deaminase

ADK, adenosine kinase

AdoMet or SAM, S-adenosylmethionine

AdoHcy, S-adenosylhomocysteine

AHCY, S-adenosylhomocysteine hydrolase or adenosylhomocysteinase

$\mathrm{AKR}$, aldo-keto reductase

APAP, acetaminophen or paracetamol

ARE, antioxidant response element

AUF1, AU-rich RNA binding factor

BHMT and BHMT2, betaine homocysteine S-methyltransferases

BSO, buthionine sulfoximine

CBS, cystathionine $\beta$-synthase

$\mathrm{CTH}$, cystathionase or cystathionine $\gamma$-lyase

cyPG, cyclopentenone prostaglandin

DNMT, DNA methyltransferase

EGSH, glutathione monoethyl ester

ER, endoplasmic reticulum

EZH2, Enhancer of Zeste Homologue 2

FXR, farnesoid responsive element

Gal, D-galactosamine

GAMT, guanidinoacetate N-methyltransferase

GCL, $\gamma$-glutamylcysteine synthetase or glutamate cysteine ligase

GNMT, glycine N-methyltransferase

GSH, glutathione reduced form 
Pajares and Pérez-Sala

GSR, glutathione reductase

GSS, glutathione synthetase

GSSG, glutathione oxidized form

GST, glutathione S-transferase

$\mathrm{H}_{2} \mathrm{~S}$, hydrogen sulfide

$\mathrm{H} 3 \mathrm{~K} 27 \mathrm{me} 3, \mathrm{H} 3 \mathrm{~K} 4 \mathrm{me} 2$ and $\mathrm{H} 3 \mathrm{~K} 9 \mathrm{me} 2$, histone 3 trimethylation or demethylation in lysines 27,4 or 9

HCC, hepatocellular carcinoma

HDAC, histone deacetylase

HO-1, heme oxygenase

Hcy, homocysteine

HHcy, hyperhomocysteinemia

HMGB1, high-mobility group box 1

HNE, 4-hydroxynonenal

HPRT, hypoxanthine phosphoribosyltransferase

HuR, Hu antigen R or ELAVL1

LEC, Long Evans Cinnamon

MAT, methionine adenosyltransferase

MAT $\alpha 1$, methionine adenosyltransferase catalytic subunit $\alpha 1$

MAT $\alpha 2$, methionine adenosyltransferase catalytic subunit $\alpha 2$

MAT $\beta$, methionine adenosyltransferase regulatory subunit

6-MP, 6-mercaptopurine

MTA, methylthioadenosine

MTR, methionine synthase

MTHF, 5-methyltetrahydrofolate 
Pajares and Pérez-Sala

MTHFR, methylenetetrahydrofolate reductase

NAC, N-acetylcysteine

NNMT, nicotinamide N-methyltransferase

NO, nitric oxide

OGlcNAc, O-linked $\mathrm{N}$-acetylglucosamine incorporation

$\mathrm{PAH}, 4 \mathrm{~S}$ polycyclic aromatic hydrocarbon

PC, phosphatidylcholine

PDRG1, p53 and DNA damage-regulated gene 1

PEMT, phosphatidylethanolamine N-methyltransferase

PHB1, prohibitin 1

PKA, PKC and PKG, protein kinases $\mathrm{A}, \mathrm{C}$ and $\mathrm{G}$

PP1 and PP2A, protein phosphatases 1 and 2A

PRC1, PRC2 and PRC3, polycomb repressive complexes 1, 2 and 3

PTM, posttranslational modification

PUFA, polyunsaturated fatty acid

ROS, reactive oxygen species

SDR, short-chain dehydrogenase/reductase

SNP, single nucleotide polymorphism

TPMT, thiopurine methyltransferase

Tx-j, toxic-milk

XRE, xenobiotic responsive element 
Pajares and Pérez-Sala

\section{REFERENCES}

1. Adler V, Yin Z, Fuchs SY, Benezra M, Rosario L, Tew KD, Pincus MR, Sardana M, Henderson CJ, Wolf CR, Davis RJ, Ronai Z. Regulation of JNK signaling by GSTp. EMBO J 18: 1321-1334, 1999.

2. Agrimi G, Di Noia MA, Marobbio CM, Fiermonte G, Lasorsa FM, Palmieri F. Identification of the human mitochondrial S-adenosylmethionine transporter: bacterial expression, reconstitution, functional characterization and tissue distribution. Biochem $J$ 379: 183-190, 2004.

3. Aguilar TS, Benevenga NJ, Harper AE. Effect of dietary methionine level on its metabolism in rats. J Nutr 104: 761-771, 1974.

4. Aissa AF, Tryndyak V, de Conti A, Melnyk S, Gomes TD, Bianchi ML, James SJ, Beland FA, Antunes LM, Pogribny IP. Effect of methionine-deficient and methionine-supplemented diets on the hepatic one-carbon and lipid metabolism in mice. Mol Nutr Food Res 58: 1502-1512, 2014.

5. Akerboom TP, Bilzer M, Sies H. The relationship of biliary glutathione disulfide efflux and intracellular glutathione disulfide content in perfused rat liver. J Biol Chem 257: 4248-4252, 1982.

6. Albers E. Metabolic characteristics and importance of the universal methionine salvage pathway recycling methionine from 5'-methylthioadenosine. IUBMB Life 61: 1132-1142, 2009.

7. Alcaide P, Merinero B, Ruiz-Sala P, Richard E, Navarrete R, Arias A, Ribes A, Artuch R, Campistol J, Ugarte M, Rodriguez-Pombo P. Defining the pathogenicity of creatine deficiency syndrome. Hum Mutat 32: 282-291, 2011. 
Pajares and Pérez-Sala

8. Alekseyenko AA, Gorchakov AA, Kharchenko PV, Kuroda MI. Reciprocal interactions of human C10orf12 and C17orf96 with PRC2 revealed by BioTAP-XL cross-linking and affinity purification. Proc Natl Acad Sci U S A 111: 2488-2493, 2014.

9. Alemany S, Varela I, Mato JM. Stimulation by vasopressin and angiotensin of phospholipid methyltransferase in isolated rat hepatocytes. FEBS Lett 135: 111-114, 1981.

10. Alvarez L, Sanchez-Gongora E, Mingorance J, Pajares MA, Mato JM. Characterization of rat liver-specific methionine adenosyltransferase gene promoter. Role of distal upstream cis-acting elements in the regulation of the transcriptional activity. J Biol Chem 272: 22875-22883, 1997.

11. Ansari A, Patel N, Sanderson J, O'Donohue J, Duley JA, Florin TH. Low-dose azathioprine or mercaptopurine in combination with allopurinol can bypass many adverse drug reactions in patients with inflammatory bowel disease. Aliment Pharmacol Ther 31: 640-647, 2010.

12. Avila MA, Berasain C, Torres L, Martin-Duce A, Corrales FJ, Yang H, Prieto J, Lu SC, Caballeria J, Rodes J, Mato JM. Reduced mRNA abundance of the main enzymes involved in methionine metabolism in human liver cirrhosis and hepatocellular carcinoma. J Hepatol 33: 907-914, 2000.

13. Avila MA, Mingorance J, Martinez-Chantar ML, Casado M, Martin-Sanz P, Bosca L, Mato JM. Regulation of rat liver S-adenosylmethionine synthetase during septic shock: role of nitric oxide. Hepatology 25: 391-396, 1997.

14. Baba SP, Wetzelberger K, Hoetker JD, Bhatnagar A. Posttranslational glutathiolation of aldose reductase (AKR1B1): a possible mechanism of protein recovery from S-nitrosylation. Chem Biol Interact 178: 250-258, 2009. 
Pajares and Pérez-Sala

15. Banerjee R. Catalytic promiscuity and heme-dependent redox regulation of $\mathrm{H} 2 \mathrm{~S}$ synthesis. Curr Opin Chem Biol 37: 115-121, 2017.

16. Bao L, Vlcek C, Paces V, Kraus JP. Identification and tissue distribution of human cystathionine beta-synthase mRNA isoforms. Arch Biochem Biophys 350: 95103, 1998.

17. Baric I, Fumic K, Glenn B, Cuk M, Schulze A, Finkelstein JD, James SJ, Mejaski-Bosnjak V, Pazanin L, Pogribny IP, Rados M, Sarnavka V, Scukanec-Spoljar M, Allen RH, Stabler S, Uzelac L, Vugrek O, Wagner C, Zeisel S, Mudd SH. Sadenosylhomocysteine hydrolase deficiency in a human: a genetic disorder of methionine metabolism. Proc Natl Acad Sci US A 101: 4234-4239, 2004.

18. Baumann E. Übel cystin und cystein. Z Physiol Chem 8: 299-305, 1884.

19. Beluzic R, Cuk M, Pavkov T, Fumic K, Baric I, Mudd SH, Jurak I, Vugrek O. A single mutation at Tyr143 of human S-adenosylhomocysteine hydrolase renders the enzyme thermosensitive and affects the oxidation state of bound cofactor nicotinamideadenine dinucleotide. Biochem J 400: 245-253, 2006.

20. Bernardini S, Bernassola F, Cortese C, Ballerini S, Melino G, Motti C, Bellincampi L, Iori R, Federici G. Modulation of GST P1-1 activity by polymerization during apoptosis. $J$ Cell Biochem 77: 645-653, 2000.

21. Bertheloot D, Latz E. HMGB1, IL-1alpha, IL-33 and S100 proteins: dualfunction alarmins. Cell Mol Immunol 14: 43-64, 2017.

22. Bestor TH, Ingram VM. Two DNA methyltransferases from murine erythroleukemia cells: purification, sequence specificity, and mode of interaction with DNA. Proc Natl Acad Sci U S A 80: 5559-5563, 1983.

23. Bienvenut WV, Sumpton D, Martinez A, Lilla S, Espagne C, Meinnel T, Giglione C. Comparative large scale characterization of plant versus mammal proteins 
Pajares and Pérez-Sala

reveals similar and idiosyncratic N-alpha-acetylation features. Mol Cell Proteomics 11: M111 015131, 2012.

24. Bigey P, Ramchandani S, Theberge J, Araujo FD, Szyf M. Transcriptional regulation of the human DNA Methyltransferase (dnmt1) gene. Gene 242: 407-418, 2000.

25. Bischoff R, Schluter H. Amino acids: chemistry, functionality and selected nonenzymatic post-translational modifications. J Proteomics 75: 2275-2296, 2012.

26. Blaker PA, Arenas-Hernandez M, Smith MA, Shobowale-Bakre EA, Fairbanks L, Irving PM, Sanderson JD, Marinaki AM. Mechanism of allopurinol induced TPMT inhibition. Biochem Pharmacol 86: 539-547, 2013.

27. Blom HJ. Consequences of homocysteine export and oxidation in the vascular system. Semin Thromb Hemost 26: 227-232, 2000.

28. Board PG, Menon D. Glutathione transferases, regulators of cellular metabolism and physiology. Biochim Biophys Acta 1830: 3267-3288, 2013.

29. Brosnan JT, Jacobs RL, Stead LM, Brosnan ME. Methylation demand: a key determinant of homocysteine metabolism. Acta Biochim Pol 51: 405-413, 2004.

30. Brown FC. gamma-Cystathionase of rat liver: effects of pyridoxal phosphate and other compounds on reaction rates. Arch Biochem Biophys 171: 378-384, 1975.

31. Brown KD, Maqsood S, Huang JY, Pan Y, Harkcom W, Li W, Sauve A, Verdin E, Jaffrey SR. Activation of SIRT3 by the $\mathrm{NAD}(+)$ precursor nicotinamide riboside protects from noise-induced hearing loss. Cell Metab 20: 1059-1068, 2014.

32. Butz LW, du Vigneaud VJ. The formation of a homologue of cystine by the decomposition of methionine with sulfuric acid. J Biol Chem 99: 135-142, 1932. 
Pajares and Pérez-Sala

33. Cabrero C, Duce AM, Ortiz P, Alemany S, Mato JM. Specific loss of the highmolecular-weight form of S-adenosyl-L-methionine synthetase in human liver cirrhosis. Hepatology 8: 1530-1534, 1988.

34. Cabrero C, Puerta J, Alemany S. Purification and comparison of two forms of Sadenosyl-L-methionine synthetase from rat liver. Eur J Biochem 170: 299-304, 1987.

35. Cadoni G, Agostino S, Scipione S, Galli J. Low serum folate levels: a risk factor for sudden sensorineural hearing loss? Acta Otolaryngol 124: 608-611, 2004.

36. Cai J, Sun WM, Hwang JJ, Stain SC, Lu SC. Changes in S-adenosylmethionine synthetase in human liver cancer: molecular characterization and significance. Hepatology 24: 1090-1097, 1996.

37. Caldeira Araujo H, Smit W, Verhoeven NM, Salomons GS, Silva S, Vasconcelos R, Tomas H, Tavares de Almeida I, Jakobs C, Duran M. Guanidinoacetate methyltransferase deficiency identified in adults and a child with mental retardation. $\mathrm{Am}$ J Med Genet A 133A: 122-127, 2005.

38. Calvisi DF, Simile MM, Ladu S, Pellegrino R, De Murtas V, Pinna F, Tomasi ML, Frau M, Virdis P, De Miglio MR, Muroni MR, Pascale RM, Feo F. Altered methionine metabolism and global DNA methylation in liver cancer: relationship with genomic instability and prognosis. Int J Cancer 121: 2410-2420, 2007.

39. Cantoni GL. Activation of methionine for transmethylation. J Biol Chem 189: 745-754, 1951.

40. Cantoni GL. S-Adenosylmethionine; a new intermediate formed enzymatically from L-methionine and adenosinetriphosphate. J Biol Chem 204: 403-416, 1953.

41. Cantoni GL. Biological methylation: selected aspects. Annu Rev Biochem 44: 435-451, 1975. 
Pajares and Pérez-Sala

42. Cardoso C, Timsit S, Villard L, Khrestchatisky M, Fontes M, Colleaux L. Specific interaction between the XNP/ATR-X gene product and the SET domain of the human EZH2 protein. Hum Mol Genet 7: 679-684, 1998.

43. Carling D, Thornton C, Woods A, Sanders MJ. AMP-activated protein kinase: new regulation, new roles? Biochem J 445: 11-27, 2012.

44. Carson NA, Neill DW. Metabolic abnormalities detected in a survey of mentally backward individuals in Northern Ireland. Arch Dis Child 37: 505-513, 1962.

45. Carter RN, Morton NM. Cysteine and hydrogen sulphide in the regulation of metabolism: insights from genetics and pharmacology. J Pathol 238: 321-332, 2016.

46. Casique L, Kabil O, Banerjee R, Martinez JC, De Lucca M. Characterization of two pathogenic mutations in cystathionine beta-synthase: different intracellular locations for wild-type and mutant proteins. Gene 531: 117-124, 2013.

47. Castano JG, Alemany S, Nieto A, Mato JM. Activation of phospholipid methyltransferase by glucagon in rat hepatocytes. J Biol Chem 255: 9041-9043, 1980.

48. Cha TL, Zhou BP, Xia W, Wu Y, Yang CC, Chen CT, Ping B, Otte AP, Hung MC. Akt-mediated phosphorylation of EZH2 suppresses methylation of lysine 27 in histone H3. Science 310: 306-310, 2005.

49. Chabner BA, Allegra CJ, Curt GA, Clendeninn NJ, Baram J, Koizumi S, Drake JC, Jolivet J. Polyglutamation of methotrexate. Is methotrexate a prodrug? J Clin Invest 76: 907-912, 1985.

50. Chacko G, Ling Q, Hajjar KA. Induction of acute translational response genes by homocysteine. Elongation factors-1alpha, -beta, and -delta. J Biol Chem 273: 1984019846, 1998. 
Pajares and Pérez-Sala

51. Chamberlin ME, Ubagai T, Mudd SH, Levy HL, Chou JY. Dominant inheritance of isolated hypermethioninemia is associated with a mutation in the human methionine adenosyltransferase 1A gene. Am J Hum Genet 60: 540-546, 1997.

52. Chasse JF, Paul V, Escanez R, Kamoun P, London J. Human cystathionine betasynthase: gene organization and expression of different $5^{\prime}$ alternative splicing. Mamm Genome 8: 917-921, 1997.

53. Chen H, Rossier C, Antonarakis SE. Cloning of a human homolog of the Drosophila enhancer of zeste gene (EZH2) that maps to chromosome 21q22.2. Genomics 38: 30-37, 1996.

54. Chen NC, Yang F, Capecci LM, Gu Z, Schafer AI, Durante W, Yang XF, Wang H. Regulation of homocysteine metabolism and methylation in human and mouse tissues. FASEB J 24: 2804-2817, 2010.

55. Chen S, Bohrer LR, Rai AN, Pan Y, Gan L, Zhou X, Bagchi A, Simon JA, Huang H. Cyclin-dependent kinases regulate epigenetic gene silencing through phosphorylation of EZH2. Nat Cell Biol 12: 1108-1114, 2010.

56. Chen X, Wang L, Fazlieva R, Kruger WD. Contrasting behaviors of mutant cystathionine beta-synthase enzymes associated with pyridoxine response. Hum Mutat 27: 474-482, 2006.

57. Chiang PK. Biological effects of inhibitors of S-adenosylhomocysteine hydrolase. Pharmacol Ther 77: 115-134, 1998.

58. Chiba T, Suzuki E, Negishi M, Saraya A, Miyagi S, Konuma T, Tanaka S, Tada M, Kanai F, Imazeki F, Iwama A, Yokosuka O. 3-Deazaneplanocin A is a promising therapeutic agent for the eradication of tumor-initiating hepatocellular carcinoma cells. Int J Cancer 130: 2557-2567, 2012. 
Pajares and Pérez-Sala

59. Chu CS, Lo PW, Yeh YH, Hsu PH, Peng SH, Teng YC, Kang ML, Wong CH, Juan LJ. O-GlcNAcylation regulates EZH2 protein stability and function. Proc Natl Acad Sci U S A 111: 1355-1360, 2014.

60. Clarke SG. 16 Inhibition of mammalian protein methyltransferases by 5'methylthioadenosine (MTA): A mechanism of action of dietary same? Enzymes 24: 467-493, 2006.

61. Cohen-Salmon M, Regnault B, Cayet N, Caille D, Demuth K, Hardelin JP, Janel N, Meda P, Petit C. Connexin30 deficiency causes instrastrial fluid-blood barrier disruption within the cochlear stria vascularis. Proc Natl Acad Sci U S A 104: 62296234, 2007.

62. Cole LK, Vance DE. A role for Sp1 in transcriptional regulation of phosphatidylethanolamine N-methyltransferase in liver and 3T3-L1 adipocytes. $J$ Biol Chem 285: 11880-11891, 2010.

63. Colell A, Garcia-Ruiz C, Morales A, Ballesta A, Ookhtens M, Rodes J, Kaplowitz N, Fernandez-Checa JC. Transport of reduced glutathione in hepatic mitochondria and mitoplasts from ethanol-treated rats: effect of membrane physical properties and S-adenosyl-L-methionine. Hepatology 26: 699-708, 1997.

64. Corrales F, Gimenez A, Alvarez L, Caballeria J, Pajares MA, Andreu H, Pares A, Mato JM, Rodes J. S-adenosylmethionine treatment prevents carbon tetrachlorideinduced S-adenosylmethionine synthetase inactivation and attenuates liver injury. Hepatology 16: 1022-1027, 1992.

65. Corrales F, Ochoa P, Rivas C, Martin-Lomas M, Mato JM, Pajares MA. Inhibition of glutathione synthesis in the liver leads to S-adenosyl-L-methionine synthetase reduction. Hepatology 14: 528-533, 1991.

66. Craig SA. Betaine in human nutrition. Am J Clin Nutr 80: 539-549, 2004. 
Pajares and Pérez-Sala

67. d'Emmanuele di Villa Bianca R, Mitidieri E, Fusco F, Russo A, Pagliara V, Tramontano T, Donnarumma E, Mirone V, Cirino G, Russo G, Sorrentino R. Urothelium muscarinic activation phosphorylates CBS(Ser227) via cGMP/PKG pathway causing human bladder relaxation through H2S production. Sci Rep 6: 31491, 2016.

68. da Silva VR, Ralat MA, Quinlivan EP, DeRatt BN, Garrett TJ, Chi YY, Frederik Nijhout H, Reed MC, Gregory JF, 3rd. Targeted metabolomics and mathematical modeling demonstrate that vitamin B-6 restriction alters one-carbon metabolism in cultured HepG2 cells. Am J Physiol Endocrinol Metab 307: E93-101, 2014.

69. da Silva VR, Rios-Avila L, Lamers Y, Ralat MA, Midttun O, Quinlivan EP, Garrett TJ, Coats B, Shankar MN, Percival SS, Chi YY, Muller KE, Ueland PM, Stacpoole PW, Gregory JF, 3rd. Metabolite profile analysis reveals functional effects of 28-day vitamin B-6 restriction on one-carbon metabolism and tryptophan catabolic pathways in healthy men and women. J Nutr 143: 1719-1727, 2013.

70. De La Haba G, Cantoni GL. The enzymatic synthesis of S-adenosyl-Lhomocysteine from adenosine and homocysteine. J Biol Chem 234: 603-608, 1959.

71. Delgado M, Garrido F, Perez-Miguelsanz J, Pacheco M, Partearroyo T, PerezSala D, Pajares MA. Acute liver injury induces nucleocytoplasmic redistribution of hepatic methionine metabolism enzymes. Antioxid Redox Signal 20: 2541-2554, 2014.

72. Delgado M, Perez-Miguelsanz J, Garrido F, Rodriguez-Tarduchy G, Perez-Sala D, Pajares MA. Early effects of copper accumulation on methionine metabolism. Cell Mol Life Sci 65: 2080-2090, 2008.

73. Demaine AG. Polymorphisms of the aldose reductase gene and susceptibility to diabetic microvascular complications. Curr Med Chem 10: 1389-1398, 2003. 
Pajares and Pérez-Sala

74. Deplus R, Blanchon L, Rajavelu A, Boukaba A, Defrance M, Luciani J, Rothe F, Dedeurwaerder S, Denis H, Brinkman AB, Simmer F, Muller F, Bertin B, Berdasco M, Putmans P, Calonne E, Litchfield DW, de Launoit Y, Jurkowski TP, Stunnenberg HG, Bock C, Sotiriou C, Fraga MF, Esteller M, Jeltsch A, Fuks F. Regulation of DNA methylation patterns by CK2-mediated phosphorylation of Dnmt3a. Cell Rep 8: 743$753,2014$.

75. Deplus R, Denis H, Putmans P, Calonne E, Fourrez M, Yamamoto K, Suzuki A, Fuks F. Citrullination of DNMT3A by PADI4 regulates its stability and controls DNA methylation. Nucleic Acids Res 42: 8285-8296, 2014.

76. DeRatt BN, Ralat MA, Kabil O, Chi YY, Banerjee R, Gregory JF, 3rd. Vitamin B-6 restriction reduces the production of hydrogen sulfide and its biomarkers by the transsulfuration pathway in cultured human hepatoma cells. J Nutr 144: 1501-1508, 2014.

77. Devogelaere B, Sammels E, De Smedt H. The IRBIT domain adds new functions to the AHCY family. Bioessays 30: 642-652, 2008.

78. Diala ES, Hoffman RM. Hypomethylation of HeLa cell DNA and the absence of 5-methylcytosine in SV40 and adenovirus (type 2) DNA: analysis by HPLC. Biochem Biophys Res Commun 107: 19-26, 1982.

79. Diez-Dacal B, Gayarre J, Gharbi S, Timms JF, Coderch C, Gago F, Perez-Sala D. Identification of aldo-keto reductase AKR1B10 as a selective target for modification and inhibition by prostaglandin A(1): implications for antitumoral activity. Cancer Res 71: 4161-4171, 2011.

80. Diez-Dacal B, Sanchez-Gomez FJ, Sanchez-Murcia PA, Milackova I, Zimmerman T, Ballekova J, Garcia-Martin E, Agundez JA, Gharbi S, Gago F, Stefek M, Perez-Sala D. Molecular Interactions and Implications of Aldose Reductase 
Pajares and Pérez-Sala

Inhibition by PGA1 and Clinically Used Prostaglandins. Mol Pharmacol 89: 42-52, 2016.

81. Ding N, Bonham EM, Hannon BE, Amick TR, Baylin SB, O'Hagan HM. Mismatch repair proteins recruit DNA methyltransferase 1 to sites of oxidative DNA damage. J Mol Cell Biol 8: 244-254, 2016.

82. Doorn JA, Srivastava SK, Petersen DR. Aldose reductase catalyzes reduction of the lipid peroxidation product 4-oxonon-2-enal. Chem Res Toxicol 16: 1418-1423, 2003.

83. Dubinsky MC, Lamothe S, Yang HY, Targan SR, Sinnett D, Theoret Y, Seidman EG. Pharmacogenomics and metabolite measurement for 6-mercaptopurine therapy in inflammatory bowel disease. Gastroenterology 118: 705-713, 2000.

84. Duce AM, Ortiz P, Cabrero C, Mato JM. S-adenosyl-L-methionine synthetase and phospholipid methyltransferase are inhibited in human cirrhosis. Hepatology 8: 6568, 1988.

85. Dullemeijer C, Verhoef P, Brouwer IA, Kok FJ, Brummer RJ, Durga J. Plasma very long-chain n-3 polyunsaturated fatty acids and age-related hearing loss in older adults. J Nutr Health Aging 14: 347-351, 2010.

86. Duncan TM, Reed MC, Nijhout HF. The relationship between intracellular and plasma levels of folate and metabolites in the methionine cycle: a model. Mol Nutr Food Res 57: 628-636, 2013.

87. Durga J, Verhoef P, Anteunis LJ, Schouten E, Kok FJ. Effects of folic acid supplementation on hearing in older adults: a randomized, controlled trial. Ann Intern Med 146: 1-9, 2007.

88. Eagle H. Nutrition needs of mammalian cells in tissue culture. Science 122: 501$514,1955$. 
Pajares and Pérez-Sala

89. Ereno-Orbea J, Majtan T, Oyenarte I, Kraus JP, Martinez-Cruz LA. Structural basis of regulation and oligomerization of human cystathionine beta-synthase, the central enzyme of transsulfuration. Proc Natl Acad Sci U S A 110: E3790-3799, 2013.

90. Ericson LE, Williams JN, Jr., Elvehjem CA. Studies on partially purified betaine-homocysteine transmethylase of liver. J Biol Chem 212: 537-544, 1955.

91. Espinos C, Garcia-Cazorla A, Martinez-Rubio D, Martinez-Martinez E, Vilaseca MA, Perez-Duenas B, Kozich V, Palau F, Artuch R. Ancient origin of the CTH alelle carrying the c.200C > T (p.T67I) variant in patients with cystathioninuria. Clin Genet 78: $554-559,2010$.

92. Esteve PO, Chang Y, Samaranayake M, Upadhyay AK, Horton JR, Feehery GR, Cheng X, Pradhan S. A methylation and phosphorylation switch between an adjacent lysine and serine determines human DNMT1 stability. Nat Struct Mol Biol 18: 42-48, 2011.

93. Esteve PO, Terragni J, Deepti K, Chin HG, Dai N, Espejo A, Correa IR, Jr., Bedford MT, Pradhan S. Methyllysine reader plant homeodomain (PHD) finger protein 20-like 1 (PHF20L1) antagonizes DNA (cytosine-5) methyltransferase 1 (DNMT1) proteasomal degradation. J Biol Chem 289: 8277-8287, 2014.

94. Esteve PO, Zhang G, Ponnaluri VK, Deepti K, Chin HG, Dai N, Sagum C, Black K, Correa IR, Jr., Bedford MT, Cheng X, Pradhan S. Binding of 14-3-3 reader proteins to phosphorylated DNMT1 facilitates aberrant DNA methylation and gene expression. Nucleic Acids Res 44: 1642-1656, 2016.

95. Fan K, Li N, Qi J, Yin P, Zhao C, Wang L, Li Z, Zha X. Wnt/beta-catenin signaling induces the transcription of cystathionine-gamma-lyase, a stimulator of tumor in colon cancer. Cell Signal 26: 2801-2808, 2014. 
Pajares and Pérez-Sala

96. Fan W, Yang H, Liu T, Wang J, Li TW, Mavila N, Tang Y, Yang J, Peng H, Tu J, Annamalai A, Noureddin M, Krishnan A, Gores GJ, Martinez-Chantar ML, Mato JM, Lu SC. Prohibitin 1 suppresses liver cancer tumorigenesis in mice and human hepatocellular and cholangiocarcinoma cells. Hepatology 65: 1249-1266, 2017.

97. Fatima S, Wagstaff KM, Loveland KL, Jans DA. Interactome of the negative regulator of nuclear import BRCA1-binding protein 2. Sci Rep 5: 9459, 2015.

98. Ferraro A, Mourtzoukou D, Kosmidou V, Avlonitis S, Kontogeorgos G, Zografos G, Pintzas A. EZH2 is regulated by ERK/AKT and targets integrin alpha2 gene to control Epithelial-Mesenchymal Transition and anoikis in colon cancer cells. Int J Biochem Cell Biol 45: 243-254, 2013.

99. Finamor I, Perez S, Bressan CA, Brenner CE, Rius-Perez S, Brittes PC, Cheiran G, Rocha MI, da Veiga M, Sastre J, Pavanato MA. Chronic aspartame intake causes changes in the trans-sulphuration pathway, glutathione depletion and liver damage in mice. Redox Biol 11: 701-707, 2017.

100. Finkelstein JD, Kyle WE, Harris BJ, Martin JJ. Methionine metabolism in mammals: concentration of metabolites in rat tissues. J Nutr 112: 1011-1018, 1982.

101. Finkelstein JD, Kyle WE, Martin JL, Pick AM. Activation of cystathionine synthase by adenosylmethionine and adenosylethionine. Biochem Biophys Res Commun 66: 81-87, 1975.

102. Finkelstein JD, Martin JJ, Harris B. Effect of nicotinamide on methionine metabolism in rat liver. J Nutr 118: 829-833, 1988.

103. Gallego O, Ruiz FX, Ardevol A, Dominguez M, Alvarez R, de Lera AR, Rovira C, Farres J, Fita I, Pares X. Structural basis for the high all-trans-retinaldehyde reductase activity of the tumor marker AKR1B10. Proc Natl Acad Sci U S A 104: 20764-20769, 2007. 
Pajares and Pérez-Sala

104. Gasbarrini G, Miglio F, Corazza GR, Stefanini GF. [On the therapeutic combination of S-adenosylmethionine with D-penicillamine in Wilson's disease]. Minerva Med 66: 1600-1604, 1975.

105. Gassen NC, Fries GR, Zannas AS, Hartmann J, Zschocke J, Hafner K, CarrilloRoa T, Steinbacher J, Preissinger SN, Hoeijmakers L, Knop M, Weber F, Kloiber S, Lucae S, Chrousos GP, Carell T, Ising M, Binder EB, Schmidt MV, Ruegg J, Rein T. Chaperoning epigenetics: FKBP51 decreases the activity of DNMT1 and mediates epigenetic effects of the antidepressant paroxetine. Sci Signal 8: ra119, 2015.

106. Gayarre J, Avellano MI, Sanchez-Gomez FJ, Carrasco MJ, Canada FJ, PerezSala D. Modification of proteins by cyclopentenone prostaglandins is differentially modulated by GSH in vitro. Ann N Y Acad Sci 1096: 78-85, 2007.

107. Ge Y, Jensen TL, Matherly LH, Taub JW. Transcriptional regulation of the cystathionine-beta -synthase gene in Down syndrome and non-Down syndrome megakaryocytic leukemia cell lines. Blood 101: 1551-1557, 2003.

108. Ge Y, Konrad MA, Matherly LH, Taub JW. Transcriptional regulation of the human cystathionine beta-synthase $-1 \mathrm{~b}$ basal promoter: synergistic transactivation by transcription factors NF-Y and Sp1/Sp3. Biochem J 357: 97-105, 2001.

109. Ge Y, Matherly LH, Taub JW. Transcriptional regulation of cell-specific expression of the human cystathionine beta -synthase gene by differential binding of Sp1/Sp3 to the -1b promoter. J Biol Chem 276: 43570-43579, 2001.

110. Ghosh S, Wanders D, Stone KP, Van NT, Cortez CC, Gettys TW. A systems biology analysis of the unique and overlapping transcriptional responses to caloric restriction and dietary methionine restriction in rats. FASEB J 28: 2577-2590, 2014. 
Pajares and Pérez-Sala

111. Gil B, Casado M, Pajares MA, Bosca L, Mato JM, Martin-Sanz P, Alvarez L. Differential expression pattern of S-adenosylmethionine synthetase isoenzymes during rat liver development. Hepatology 24: 876-881, 1996.

112. Gil B, Pajares MA, Mato JM, Alvarez L. Glucocorticoid regulation of hepatic Sadenosylmethionine synthetase gene expression. Endocrinology 138: 1251-1258, 1997.

113. Gilot D, Loyer P, Corlu A, Glaise D, Lagadic-Gossmann D, Atfi A, Morel F, Ichijo H, Guguen-Guillouzo C. Liver protection from apoptosis requires both blockage of initiator caspase activities and inhibition of ASK1/JNK pathway via glutathione Stransferase regulation. J Biol Chem 277: 49220-49229, 2002.

114. Gong D, Cheng HP, Xie W, Zhang M, Liu D, Lan G, Huang C, Zhao ZW, Chen LY, Yao F, Tan YL, Li L, Xia XD, Zheng XL, Wang ZB, Tang CK. Cystathionine gamma-lyase(CSE)/hydrogen sulfide system is regulated by miR-216a and influences cholesterol efflux in macrophages via the PI3K/AKT/ABCA1 pathway. Biochem Biophys Res Commun 470: 107-116, 2016.

115. Gonzalez B, Garrido F, Ortega R, Martinez-Julvez M, Revilla-Guarinos A, Perez-Pertejo Y, Velazquez-Campoy A, Sanz-Aparicio J, Pajares MA. NADP+ binding to the regulatory subunit of methionine adenosyltransferase II increases intersubunit binding affinity in the hetero-trimer. PLoS One 7: e50329, 2012.

116. Gonzalez B, Pajares MA, Hermoso JA, Alvarez L, Garrido F, Sufrin JR, SanzAparicio J. The crystal structure of tetrameric methionine adenosyltransferase from rat liver reveals the methionine-binding site. J Mol Biol 300: 363-375, 2000.

117. Gonzalez B, Pajares MA, Hermoso JA, Guillerm D, Guillerm G, Sanz-Aparicio J. Crystal structures of methionine adenosyltransferase complexed with substrates and products reveal the methionine-ATP recognition and give insights into the catalytic mechanism. J Mol Biol 331: 407-416, 2003. 
Pajares and Pérez-Sala

118. González MP, Varela-Moreiras G, Selhub J, Paul L, Pajares MA. Methionine adenosyltransferase expression depends on methionine concentrations in the culture media. Ann Nutr Metab 63: 1342, 2013.

119. Gopinath B, Flood VM, Rochtchina E, McMahon CM, Mitchell P. Consumption of omega-3 fatty acids and fish and risk of age-related hearing loss. Am J Clin Nutr 92: 416-421, 2010.

120. Gopinath B, Flood VM, Rochtchina E, McMahon CM, Mitchell P. Serum homocysteine and folate concentrations are associated with prevalent age-related hearing loss. J Nutr 140: 1469-1474, 2010.

121. Guggenheim S, Flavin M. Cystathionine gamma-synthase. A pyridoxal phosphate enzyme catalyzing rapid exchanges of beta and alpha hydrogen atoms in amino acids. J Biol Chem 244: 6217-6227, 1969.

122. Guo DC, Gong L, Regalado ES, Santos-Cortez RL, Zhao R, Cai B, Veeraraghavan S, Prakash SK, Johnson RJ, Muilenburg A, Willing M, Jondeau G, Boileau C, Pannu H, Moran R, Debacker J, GenTac Investigators NHL, Blood Institute Go Exome Sequencing P, Montalcino Aortic C, Bamshad MJ, Shendure J, Nickerson DA, Leal SM, Raman CS, Swindell EC, Milewicz DM. MAT2A mutations predispose individuals to thoracic aortic aneurysms. Am J Hum Genet 96: 170-177, 2015.

123. Gupta RA, Yuan CS, Ault-Riche DB, Borchardt RT. Limited proteolysis of Sadenosylhomocysteine hydrolase: implications for the three-dimensional structure. Arch Biochem Biophys 319: 365-371, 1995.

124. Ha SY, Song DH, Lee JJ, Lee HW, Cho SY, Park CK. High expression of aldoketo reductase $1 \mathrm{~B} 10$ is an independent predictor of favorable prognosis in patients with hepatocellular carcinoma. Gut Liver 8: 648-654, 2014. 
Pajares and Pérez-Sala

125. Halim AB, LeGros L, Chamberlin ME, Geller A, Kotb M. Regulation of the human MAT2A gene encoding the catalytic alpha 2 subunit of methionine adenosyltransferase, MAT II: gene organization, promoter characterization, and identification of a site in the proximal promoter that is essential for its activity. $J$ Biol Chem 276: 9784-9791, 2001.

126. Halsted $\mathrm{CH}$, Villanueva JA, Devlin AM. Folate deficiency, methionine metabolism, and alcoholic liver disease. Alcohol 27: 169-172, 2002.

127. Halsted CH, Villanueva JA, Devlin AM, Niemela O, Parkkila S, Garrow TA, Wallock LM, Shigenaga MK, Melnyk S, James SJ. Folate deficiency disturbs hepatic methionine metabolism and promotes liver injury in the ethanol-fed micropig. Proc Natl Acad Sci U S A 99: 10072-10077, 2002.

128. Harris H, Penrose LS, Thomas DH. Cystathioniuria. Ann Hum Genet 23: 442453, 1959.

129. Hayakawa J, Mittal S, Wang Y, Korkmaz KS, Adamson E, English C, Ohmichi M, McClelland M, Mercola D. Identification of promoters bound by c-Jun/ATF2 during rapid large-scale gene activation following genotoxic stress. Mol Cell 16: 521-535, 2004.

130. Hazelwood S, Bernardini I, Shotelersuk V, Tangerman A, Guo J, Mudd H, Gahl WA. Normal brain myelination in a patient homozygous for a mutation that encodes a severely truncated methionine adenosyltransferase I/III. Am J Med Genet 75: 395-400, 1998.

131. Heady JE, Kerr SJ. Alteration of glycine N-methyltransferase activity in fetal, adult, and tumor tissues. Cancer Res 35: 640-643, 1975. 
Pajares and Pérez-Sala

132. Heibein AD, Guo B, Sprowl JA, Maclean DA, Parissenti AM. Role of aldo-keto reductases and other doxorubicin pharmacokinetic genes in doxorubicin resistance, DNA binding, and subcellular localization. BMC Cancer 12: 381, 2012.

133. Henderson CJ, McLaren AW, Wolf CR. In vivo regulation of human glutathione transferase GSTP by chemopreventive agents. Cancer Res 74: 4378-4387, 2014.

134. Hendriks IA, D'Souza RC, Yang B, Verlaan-de Vries M, Mann M, Vertegaal AC. Uncovering global SUMOylation signaling networks in a site-specific manner. Nat Struct Mol Biol 21: 927-936, 2014.

135. Heringlake S, Hofdmann M, Fiebeler A, Manns MP, Schmiegel W, Tannapfel A. Identification and expression analysis of the aldo-ketoreductase1-B10 gene in primary malignant liver tumours. J Hepatol 52: 220-227, 2010.

136. Hine C, Harputlugil E, Zhang Y, Ruckenstuhl C, Lee BC, Brace L, Longchamp A, Trevino-Villarreal JH, Mejia P, Ozaki CK, Wang R, Gladyshev VN, Madeo F, Mair WB, Mitchell JR. Endogenous hydrogen sulfide production is essential for dietary restriction benefits. Cell 160: 132-144, 2015.

137. Hine C, Kim HJ, Zhu Y, Harputlugil E, Longchamp A, Matos MS, Ramadoss P, Bauerle K, Brace L, Asara JM, Ozaki CK, Cheng SY, Singha S, Ahn KH, Kimmelman A, Fisher FM, Pissios P, Withers DJ, Selman C, Wang R, Yen K, Longo VD, Cohen P, Bartke A, Kopchick JJ, Miller R, Hollenberg AN, Mitchell JR. Hypothalamic-Pituitary Axis Regulates Hydrogen Sulfide Production. Cell Metab 25: 1320-1333 e5, 2017.

138. Hoffman DR, Haning JA, Cornatzer WE. Microsomal phosphatidylethanolamine methyltransferase: inhibition by S-adenosylhomocysteine. Lipids 16: 561-567, 1981.

139. Hogarth LA, Redfern CP, Teodoridis JM, Hall AG, Anderson H, Case MC, Coulthard SA. The effect of thiopurine drugs on DNA methylation in relation to TPMT expression. Biochem Pharmacol 76: 1024-1035, 2008. 
Pajares and Pérez-Sala

140. Horne DW, Holloway RS, Wagner C. Transport of S-adenosylmethionine in isolated rat liver mitochondria. Arch Biochem Biophys 343: 201-206, 1997.

141. Horowitz JH, Rypins EB, Henderson JM, Heymsfield SB, Moffitt SD, Bain RP, Chawla RK, Bleier JC, Rudman D. Evidence for impairment of transsulfuration pathway in cirrhosis. Gastroenterology 81: 668-675, 1981.

142. Howarth KD, Blood KA, Ng BL, Beavis JC, Chua Y, Cooke SL, Raby S, Ichimura K, Collins VP, Carter NP, Edwards PA. Array painting reveals a high frequency of balanced translocations in breast cancer cell lines that break in cancerrelevant genes. Oncogene 27: 3345-3359, 2008.

143. Huang Y, Li W, Su ZY, Kong AN. The complexity of the Nrf2 pathway: beyond the antioxidant response. J Nutr Biochem 26: 1401-1413, 2015.

144. Huang YC, Lee CM, Chen M, Chung MY, Chang YH, Huang WJ, Ho DM, Pan CC, Wu TT, Yang S, Lin MW, Hsieh JT, Chen YM. Haplotypes, loss of heterozygosity, and expression levels of glycine N-methyltransferase in prostate cancer. Clin Cancer Res 13: 1412-1420, 2007.

145. Hudson CA, Betschart AA, Turnlund JR, Kretsch MJ, Sauberlich HE. Protein utilization by young women consuming animal or plant protein diets at various levels of vitamin B-6 intake. Am J Clin Nutr 49: 636-640, 1989.

146. Huidobro C, Torano EG, Fernandez AF, Urdinguio RG, Rodriguez RM, Ferrero C, Martinez-Camblor P, Boix L, Bruix J, Garcia-Rodriguez JL, Varela-Rey M, Mato JM, Martinez-Chantar ML, Fraga MF. A DNA methylation signature associated with the epigenetic repression of glycine $\mathrm{N}$-methyltransferase in human hepatocellular carcinoma. J Mol Med (Berl) 91: 939-950, 2013.

147. Hutchison CA, 3rd, Chuang RY, Noskov VN, Assad-Garcia N, Deerinck TJ, Ellisman MH, Gill J, Kannan K, Karas BJ, Ma L, Pelletier JF, Qi ZQ, Richter RA, 
Pajares and Pérez-Sala

Strychalski EA, Sun L, Suzuki Y, Tsvetanova B, Wise KS, Smith HO, Glass JI, Merryman C, Gibson DG, Venter JC. Design and synthesis of a minimal bacterial genome. Science 351: aad6253, 2016.

148. Ikeda R, Nishida T, Watanabe F, Shimizu-Saito K, Asahina K, Horikawa S, Teraoka H. Involvement of CCAAT/enhancer binding protein-beta (C/EBPbeta) in epigenetic regulation of mouse methionine adenosyltransferase 1A gene expression. Int J Biochem Cell Biol 40: 1956-1969, 2008.

149. Item CB, Mercimek-Mahmutoglu S, Battini R, Edlinger-Horvat C, Stromberger C, Bodamer O, Muhl A, Vilaseca MA, Korall H, Stockler-Ipsiroglu S. Characterization of seven novel mutations in seven patients with GAMT deficiency. Hum Mutat 23: 524, 2004.

150. Jacobs RL, Zhao Y, Koonen DP, Sletten T, Su B, Lingrell S, Cao G, Peake DA, Kuo MS, Proctor SD, Kennedy BP, Dyck JR, Vance DE. Impaired de novo choline synthesis explains why phosphatidylethanolamine N-methyltransferase-deficient mice are protected from diet-induced obesity. J Biol Chem 285: 22403-22413, 2010.

151. Jacometo CB, Zhou Z, Luchini D, Correa MN, Loor JJ. Maternal supplementation with rumen-protected methionine increases prepartal plasma methionine concentration and alters hepatic mRNA abundance of 1-carbon, methionine, and transsulfuration pathways in neonatal Holstein calves. J Dairy Sci 100: 3209-3219, 2017.

152. Jakubowski H, Glowacki R. Chemical biology of homocysteine thiolactone and related metabolites. Adv Clin Chem 55: 81-103, 2011.

153. Jencks DA, Mathews RG. Allosteric inhibition of methylenetetrahydrofolate reductase by adenosylmethionine. Effects of adenosylmethionine and NADPH on the 
Pajares and Pérez-Sala

equilibrium between active and inactive forms of the enzyme and on the kinetics of approach to equilibrium. $J$ Biol Chem 262: 2485-2493, 1987.

154. Jiang L, Luo X, Shi J, Sun H, Sun Q, Sheikh MS, Huang Y. PDRG1, a novel tumor marker for multiple malignancies that is selectively regulated by genotoxic stress. Cancer Biol Ther 11: 567-573, 2011.

155. Jin X, Yang C, Fan P, Xiao J, Zhang W, Zhan S, Liu T, Wang D, Wu H. CDK5/FBW7-dependent ubiquitination and degradation of EZH2 inhibits pancreatic cancer cell migration and invasion. J Biol Chem 292: 6269-6280, 2017.

156. Jinawath A, Miyake S, Yanagisawa Y, Akiyama Y, Yuasa Y. Transcriptional regulation of the human DNA methyltransferase 3A and 3B genes by Sp3 and Sp1 zinc finger proteins. Biochem J 385: 557-564, 2005.

157. Johnsson LG, Hawkins JE, Jr. Vascular changes in the human inner ear associated with aging. Ann Otol Rhinol Laryngol 81: 364-376, 1972.

158. Joncquel-Chevalier Curt M, Voicu PM, Fontaine M, Dessein AF, Porchet N, Mention-Mulliez K, Dobbelaere D, Soto-Ares G, Cheillan D, Vamecq J. Creatine biosynthesis and transport in health and disease. Biochimie 119: 146-165, 2015.

159. Jurkowska RZ, Rajavelu A, Anspach N, Urbanke C, Jankevicius G, Ragozin S, Nellen W, Jeltsch A. Oligomerization and binding of the Dnmt3a DNA methyltransferase to parallel DNA molecules: heterochromatic localization and role of Dnmt3L. J Biol Chem 286: 24200-24207, 2011.

160. Kabil O, Banerjee R. Enzymology of H2S biogenesis, decay and signaling. Antioxid Redox Signal 20: 770-782, 2014.

161. Kabil O, Vitvitsky V, Banerjee R. Sulfur as a signaling nutrient through hydrogen sulfide. Annu Rev Nutr 34: 171-205, 2014. 
Pajares and Pérez-Sala

162. Kabil O, Zhou Y, Banerjee R. Human cystathionine beta-synthase is a target for sumoylation. Biochemistry 45: 13528-13536, 2006.

163. Kajander O, Eloranta T, Raina A. A sensitive isotopic assay method for Sadenosylhomocysteine hydrolase. Some properties of the enzyme from rat liver. Biochim Biophys Acta 438: 522-531, 1976.

164. Kaneko S, Li G, Son J, Xu CF, Margueron R, Neubert TA, Reinberg D. Phosphorylation of the PRC2 component Ezh2 is cell cycle-regulated and up-regulates its binding to ncRNA. Genes Dev 24: 2615-2620, 2010.

165. Kang ES, Park CW, Chung JH. Dnmt3b, de novo DNA methyltransferase, interacts with SUMO-1 and Ubc9 through its N-terminal region and is subject to modification by SUMO-1. Biochem Biophys Res Commun 289: 862-868, 2001.

166. Kang ES, Woo IS, Kim HJ, Eun SY, Paek KS, Kim HJ, Chang KC, Lee JH, Lee HT, Kim JH, Nishinaka T, Yabe-Nishimura C, Seo HG. Up-regulation of aldose reductase expression mediated by phosphatidylinositol 3-kinase/Akt and Nrf2 is involved in the protective effect of curcumin against oxidative damage. Free Radic Biol Med 43: 535-545, 2007.

167. Kang J, Gemberling M, Nakamura M, Whitby FG, Handa H, Fairbrother WG, Tantin D. A general mechanism for transcription regulation by Oct1 and Oct4 in response to genotoxic and oxidative stress. Genes Dev 23: 208-222, 2009.

168. Katoh Y, Ikura T, Hoshikawa Y, Tashiro S, Ito T, Ohta M, Kera Y, Noda T, Igarashi K. Methionine adenosyltransferase II serves as a transcriptional corepressor of Maf oncoprotein. Mol Cell 41: 554-566, 2011.

169. Kawamoto Y, Nakamura Y, Naito Y, Torii Y, Kumagai T, Osawa T, Ohigashi H, Satoh K, Imagawa M, Uchida K. Cyclopentenone prostaglandins as potential 
Pajares and Pérez-Sala

inducers of phase II detoxification enzymes. 15-deoxy-delta(12,14)-prostaglandin j2induced expression of glutathione S-transferases. J Biol Chem 275: 11291-11299, 2000.

170. Kera Y, Katoh Y, Ohta M, Matsumoto M, Takano-Yamamoto T, Igarashi K. Methionine adenosyltransferase II-dependent histone H3K9 methylation at the COX-2 gene locus. J Biol Chem 288: 13592-13601, 2013.

171. Kerr SJ. Competing methyltransferase systems. J Biol Chem 247: 4248-4252, 1972.

172. Kim GD, Ni J, Kelesoglu N, Roberts RJ, Pradhan S. Co-operation and communication between the human maintenance and de novo DNA (cytosine-5) methyltransferases. EMBO J 21: 4183-4195, 2002.

173. Kim SH, Park J, Choi MC, Park JH, Kim HP, Lee JH, Oh DY, Im SA, Bang YJ, Kim TY. DNA methyltransferase 3B acts as a co-repressor of the human polycomb protein $\mathrm{hPc} 2$ to repress fibroblast growth factor receptor 3 transcription. Int J Biochem Cell Biol 40: 2462-2471, 2008.

174. Kimura H. Hydrogen sulfide and polysulfides as biological mediators. Molecules 19: 16146-16157, 2014.

175. Kinsell LW, Harper HA, Barton HC, Michaels GD, Weiss HA. Rate of Disappearance From Plasma of Intravenously Administered Methionine in Patients With Liver Damage. Science 106: 589-590, 1947.

176. Kitteringham NR, Powell H, Jenkins RE, Hamlett J, Lovatt C, Elsby R, Henderson CJ, Wolf CR, Pennington SR, Park BK. Protein expression profiling of glutathione S-transferase pi null mice as a strategy to identify potential markers of resistance to paracetamol-induced toxicity in the liver. Proteomics 3: 191-207, 2003.

177. Kloor D, Osswald H. S-Adenosylhomocysteine hydrolase as a target for intracellular adenosine action. Trends Pharmacol Sci 25: 294-297, 2004. 
Pajares and Pérez-Sala

178. Kobayashi M, Yamamoto M. Molecular mechanisms activating the Nrf2-Keap1 pathway of antioxidant gene regulation. Antioxid Redox Signal 7: 385-394, 2005.

179. Kolb NS, Hunsaker LA, Vander Jagt DL. Aldose reductase-catalyzed reduction of acrolein: implications in cyclophosphamide toxicity. Mol Pharmacol 45: 797-801, 1994.

180. Komoto J, Huang Y, Takata Y, Yamada T, Konishi K, Ogawa H, Gomi T, Fujioka M, Takusagawa F. Crystal structure of guanidinoacetate methyltransferase from rat liver: a model structure of protein arginine methyltransferase. J Mol Biol 320: 223$235,2002$.

181. Kong X, Chen L, Jiao L, Jiang X, Lian F, Lu J, Zhu K, Du D, Liu J, Ding H, Zhang N, Shen J, Zheng M, Chen K, Liu X, Jiang H, Luo C. Astemizole arrests the proliferation of cancer cells by disrupting the EZH2-EED interaction of polycomb repressive complex 2. J Med Chem 57: 9512-9521, 2014.

182. Konishi K, Fujioka M. Rat liver glycine methyltransferase. Cooperative binding of S-adenosylmethionine and loss of cooperativity by removal of a short NH2-terminal segment. J Biol Chem 263: 13381-13385, 1988.

183. Kotb M, Mudd SH, Mato JM, Geller AM, Kredich NM, Chou JY, Cantoni GL. Consensus nomenclature for the mammalian methionine adenosyltransferase genes and gene products. Trends Genet 13: 51-52, 1997.

184. Koturbash I, Melnyk S, James SJ, Beland FA, Pogribny IP. Role of epigenetic and miR-22 and miR-29b alterations in the downregulation of Matla and Mthfr genes in early preneoplastic livers in rats induced by 2-acetylaminofluorene. Mol Carcinog 52: 318-327, 2013.

185. Kovatscheva EG, Popova JG. [S-Methylmethionine content in plant and animal tissues and stability during storage]. Nahrung 21: 465-472, 1977. 
Pajares and Pérez-Sala

186. Kraus JP, Hasek J, Kozich V, Collard R, Venezia S, Janosikova B, Wang J, Stabler SP, Allen RH, Jakobs C, Finn CT, Chien YH, Hwu WL, Hegele RA, Mudd SH. Cystathionine gamma-lyase: Clinical, metabolic, genetic, and structural studies. Mol Genet Metab 97: 250-259, 2009.

187. Krupenko NI, Wagner C. Transport of rat liver glycine N-methyltransferase into rat liver nuclei. J Biol Chem 272: 27140-27146, 1997.

188. Kuchino Y, Yasuda T, Nishimura S. Deficiency of S-adenosylmethioninehomocysteine methyltransferase activity in hepatoma cells. Cancer Res 37: 206-208, 1977.

189. Kumar D, Lassar AB. Fibroblast growth factor maintains chondrogenic potential of limb bud mesenchymal cells by modulating DNMT3A recruitment. Cell Rep 8: 1419-1431, 2014.

190. Kundu S, Munjal C, Tyagi N, Sen U, Tyagi AC, Tyagi SC. Folic acid improves inner ear vascularization in hyperhomocysteinemic mice. Hear Res 284: 42-51, 2012.

191. Kuzmichev A, Nishioka K, Erdjument-Bromage H, Tempst P, Reinberg D. Histone methyltransferase activity associated with a human multiprotein complex containing the Enhancer of Zeste protein. Genes Dev 16: 2893-2905, 2002.

192. Laffin B, Petrash JM. Expression of the Aldo-Ketoreductases AKR1B1 and AKR1B10 in Human Cancers. Front Pharmacol 3: 104, 2012.

193. Laird PW, Jaenisch R. DNA methylation and cancer. Hum Mol Genet 3 Spec No: 1487-1495, 1994.

194. Landgraf BJ, McCarthy EL, Booker SJ. Radical S-Adenosylmethionine Enzymes in Human Health and Disease. Annu Rev Biochem 85: 485-514, 2016.

195. Lasisi AO, Fehintola FA, Yusuf OB. Age-related hearing loss, vitamin B12, and folate in the elderly. Otolaryngol Head Neck Surg 143: 826-830, 2010. 
Pajares and Pérez-Sala

196. Lau A, Villeneuve NF, Sun Z, Wong PK, Zhang DD. Dual roles of Nrf2 in cancer. Pharmacol Res 58: 262-270, 2008.

197. Le A, Shibata NM, French SW, Kim K, Kharbanda KK, Islam MS, LaSalle JM, Halsted CH, Keen CL, Medici V. Characterization of timed changes in hepatic copper concentrations, methionine metabolism, gene expression, and global DNA methylation in the Jackson toxic milk mouse model of Wilson disease. Int J Mol Sci 15: 8004-8023, 2014.

198. Leal JF, Ferrer I, Blanco-Aparicio C, Hernandez-Losa J, Ramon YCS, Carnero A, Lleonart ME. S-adenosylhomocysteine hydrolase downregulation contributes to tumorigenesis. Carcinogenesis 29: 2089-2095, 2008.

199. Lee B, Muller MT. SUMOylation enhances DNA methyltransferase 1 activity. Biochem J 421: 449-461, 2009.

200. Lee CM, Shih YP, Wu CH, Chen YM. Characterization of the 5' regulatory region of the human Glycine N-methyltransferase gene. Gene 443: 151-157, 2009.

201. Lee CM, Yen CH, Tzeng TY, Huang YZ, Chou KH, Chang TJ, Arthur Chen YM. Androgen response element of the glycine N-methyltransferase gene is located in the coding region of its first exon. Biosci Rep 33: e00070, 2013.

202. LeGros HL, Jr., Geller AM, Kotb M. Differential regulation of methionine adenosyltransferase in superantigen and mitogen stimulated human T lymphocytes. $J$ Biol Chem 272: 16040-16047, 1997.

203. LeGros HL, Jr., Halim AB, Geller AM, Kotb M. Cloning, expression, and functional characterization of the beta regulatory subunit of human methionine adenosyltransferase (MAT II). J Biol Chem 275: 2359-2366, 2000. 
Pajares and Pérez-Sala

204. LeGros L, Halim AB, Chamberlin ME, Geller A, Kotb M. Regulation of the human MAT2B gene encoding the regulatory beta subunit of methionine adenosyltransferase, MAT II. J Biol Chem 276: 24918-24924, 2001.

205. Li CH, Yen CH, Chen YF, Lee KJ, Fang CC, Zhang X, Lai CC, Huang SF, Lin HK, Arthur Chen YM. Characterization of the GNMT-HectH9-PREX2 Tripartite Relationship in the Pathogenesis of Hepatocellular Carcinoma. Int J Cancer 140: 2284$2297,2017$.

206. Li D, Bi FF, Chen NN, Cao JM, Sun WP, Zhou YM, Cao C, Li CY, Yang Q. Epigenetic repression of phosphatidylethanolamine N-methyltransferase (PEMT) in BRCA1-mutated breast cancer. Oncotarget 5: 1315-1325, 2014.

207. Li D, Tian YJ, Guo J, Sun WP, Lun YZ, Guo M, Luo N, Cao Y, Cao JM, Gong XJ, Zhou SS. Nicotinamide supplementation induces detrimental metabolic and epigenetic changes in developing rats. Br J Nutr 110: 2156-2164, 2013.

208. Li JJ, Li Q, Du HP, Wang YL, You SJ, Wang F, Xu XS, Cheng J, Cao YJ, Liu CF, Hu LF. Homocysteine Triggers Inflammatory Responses in Macrophages through Inhibiting CSE-H2S Signaling via DNA Hypermethylation of CSE Promoter. Int $J$ Mol Sci 16: 12560-12577, 2015.

209. Li M, Li Y, Chen J, Wei W, Pan X, Liu J, Liu Q, Leu W, Zhang L, Yang X, Lu J, Wang K. Copper ions inhibit S-adenosylhomocysteine hydrolase by causing dissociation of NAD+ cofactor. Biochemistry 46: 11451-11458, 2007.

210. Li Q, Su Z, Xu X, Liu G, Song X, Wang R, Sui X, Liu T, Chang X, Huang D. AS1DHRS4, a head-to-head natural antisense transcript, silences the DHRS4 gene cluster in cis and trans. Proc Natl Acad Sci US A 109: 14110-14115, 2012.

211. Li Z, Hou P, Fan D, Dong M, Ma M, Li H, Yao R, Li Y, Wang G, Geng P, Mihretab A, Liu D, Zhang Y, Huang B, Lu J. The degradation of EZH2 mediated by 
Pajares and Pérez-Sala

lncRNA ANCR attenuated the invasion and metastasis of breast cancer. Cell Death Differ 24: 59-71, 2017.

212. Liu HH, Lu P, Guo Y, Farrell E, Zhang X, Zheng M, Bosano B, Zhang Z, Allard J, Liao G, Fu S, Chen J, Dolim K, Kuroda A, Usuka J, Cheng J, Tao W, Welch K, Liu Y, Pease J, de Keczer SA, Masjedizadeh M, Hu JS, Weller P, Garrow T, Peltz G. An integrative genomic analysis identifies Bhmt2 as a diet-dependent genetic factor protecting against acetaminophen-induced liver toxicity. Genome Res 20: 28-35, 2010.

213. Loenen WA. S-adenosylmethionine: jack of all trades and master of everything? Biochem Soc Trans 34: 330-333, 2006.

214. Lopez-Sanchez LM, Corrales FJ, Barcos M, Espejo I, Munoz-Castaneda JR, Rodriguez-Ariza A. Inhibition of nitric oxide synthesis during induced cholestasis ameliorates hepatocellular injury by facilitating S-nitrosothiol homeostasis. Lab Invest 90: 116-127, 2010.

215. Lu SC, Alvarez L, Huang ZZ, Chen L, An W, Corrales FJ, Avila MA, Kanel G, Mato JM. Methionine adenosyltransferase 1A knockout mice are predisposed to liver injury and exhibit increased expression of genes involved in proliferation. Proc Natl Acad Sci U S A 98: 5560-5565, 2001.

216. Lu SC, Gukovsky I, Lugea A, Reyes CN, Huang ZZ, Chen L, Mato JM, Bottiglieri T, Pandol SJ. Role of S-adenosylmethionine in two experimental models of pancreatitis. FASEB J 17: 56-58, 2003.

217. Lu SC, Huang ZZ, Yang H, Mato JM, Avila MA, Tsukamoto H. Changes in methionine adenosyltransferase and S-adenosylmethionine homeostasis in alcoholic rat liver. Am J Physiol Gastrointest Liver Physiol 279: G178-185, 2000.

218. Lu SC, Mato JM. S-adenosylmethionine in liver health, injury, and cancer. Physiol Rev 92: 1515-1542, 2012. 
Pajares and Pérez-Sala

219. Luka Z, Cerone R, Phillips JA, 3rd, Mudd HS, Wagner C. Mutations in human glycine N-methyltransferase give insights into its role in methionine metabolism. Hum Genet 110: 68-74, 2002.

220. Luka Z, Ham AJ, Norris JL, Yeo EJ, Yermalitsky V, Glenn B, Caprioli RM, Liebler DC, Wagner C. Identification of phosphorylation sites in glycine Nmethyltransferase from rat liver. Protein Sci 15: 785-794, 2006.

221. Luka Z, Loukachevitch LV, Wagner C. Acetylation of N-terminal valine of glycine N-methyltransferase affects enzyme inhibition by folate. Biochim Biophys Acta 1784: 1342-1346, 2008.

222. Luka Z, Pakhomova S, Luka Y, Newcomer ME, Wagner C. Destabilization of human glycine N-methyltransferase by H176N mutation. Protein Sci 16: 1957-1964, 2007.

223. Luka Z, Wagner C. Effect of naturally occurring mutations in human glycine Nmethyltransferase on activity and conformation. Biochem Biophys Res Commun 312: 1067-1072, 2003.

224. Lyon RC, Li D, McGarvie G, Ellis EM. Aldo-keto reductases mediate constitutive and inducible protection against aldehyde toxicity in human neuroblastoma SH-SY5Y cells. Neurochem Int 62: 113-121, 2013.

225. Ma J, Yan R, Zu X, Cheng JM, Rao K, Liao DF, Cao D. Aldo-keto reductase family 1 B10 affects fatty acid synthesis by regulating the stability of acetyl-CoA carboxylase-alpha in breast cancer cells. J Biol Chem 283: 3418-3423, 2008.

226. Maclean KN, Kraus E, Kraus JP. The dominant role of Sp1 in regulating the cystathionine beta-synthase $-1 \mathrm{a}$ and $-1 \mathrm{~b}$ promoters facilitates potential tissue-specific regulation by Kruppel-like factors. J Biol Chem 279: 8558-8566, 2004. 
Pajares and Pérez-Sala

227. Maddocks OD, Labuschagne CF, Adams PD, Vousden KH. Serine Metabolism Supports the Methionine Cycle and DNA/RNA Methylation through De Novo ATP Synthesis in Cancer Cells. Mol Cell 61: 210-221, 2016.

228. Markham GD, Pajares MA. Structure-function relationships in methionine adenosyltransferases. Cell Mol Life Sci 66: 636-648, 2009.

229. Marsh S, Van Booven DJ. The increasing complexity of mercaptopurine pharmacogenomics. Clin Pharmacol Ther 85: 139-141, 2009.

230. Martin HJ, Maser E. Role of human aldo-keto-reductase AKR1B10 in the protection against toxic aldehydes. Chem Biol Interact 178: 145-150, 2009.

231. Martinez M, Cuskelly GJ, Williamson J, Toth JP, Gregory JF, 3rd. Vitamin B-6 deficiency in rats reduces hepatic serine hydroxymethyltransferase and cystathionine beta-synthase activities and rates of in vivo protein turnover, homocysteine remethylation and transsulfuration. J Nutr 130: 1115-1123, 2000.

232. Martinez-Chantar ML, Garcia-Trevijano ER, Latasa MU, Martin-Duce A, Fortes P, Caballeria J, Avila MA, Mato JM. Methionine adenosyltransferase II beta subunit gene expression provides a proliferative advantage in human hepatoma. Gastroenterology 124: 940-948, 2003.

233. Martinez-Chantar ML, Latasa MU, Varela-Rey M, Lu SC, Garcia-Trevijano ER, Mato JM, Avila MA. L-methionine availability regulates expression of the methionine adenosyltransferase $2 \mathrm{~A}$ gene in human hepatocarcinoma cells: role of $\mathrm{S}$ adenosylmethionine. J Biol Chem 278: 19885-19890, 2003.

234. Martinez-Chantar ML, Pajares MA. Role of thioltransferases on the modulation of rat liver S-adenosylmethionine synthetase activity by glutathione. FEBS Lett 397: 293-297, 1996. 
Pajares and Pérez-Sala

235. Martinez-Chantar ML, Pajares MA. Assignment of a single disulfide bridge in rat liver methionine adenosyltransferase. Eur J Biochem 267: 132-137, 2000.

236. Martinez-Chantar ML, Vazquez-Chantada M, Ariz U, Martinez N, Varela M, Luka Z, Capdevila A, Rodriguez J, Aransay AM, Matthiesen R, Yang H, Calvisi DF, Esteller M, Fraga M, Lu SC, Wagner C, Mato JM. Loss of the glycine Nmethyltransferase gene leads to steatosis and hepatocellular carcinoma in mice. Hepatology 47: 1191-1199, 2008.

237. Martinez-Chantar ML, Vazquez-Chantada M, Garnacho M, Latasa MU, VarelaRey M, Dotor J, Santamaria M, Martinez-Cruz LA, Parada LA, Lu SC, Mato JM. Sadenosylmethionine regulates cytoplasmic HuR via AMP-activated kinase. Gastroenterology 131: 223-232, 2006.

238. Martinez-Vega R, Garrido F, Partearroyo T, Cediel R, Zeisel SH, MartinezAlvarez C, Varela-Moreiras G, Varela-Nieto I, Pajares MA. Folic acid deficiency induces premature hearing loss through mechanisms involving cochlear oxidative stress and impairment of homocysteine metabolism. FASEB J 29: 418-432, 2015.

239. Martinez-Vega R, Murillo-Cuesta S, Partearroyo T, Varela-Moreiras G, VarelaNieto I, Pajares MA. Long-Term Dietary Folate Deficiency Accelerates Progressive Hearing Loss on CBA/Ca Mice. Front Aging Neurosci 8: 209, 2016.

240. Martinez-Vega R, Partearroyo T, Vallecillo N, Varela-Moreiras G, Pajares MA, Varela-Nieto I. Long-term omega-3 fatty acid supplementation prevents expression changes in cochlear homocysteine metabolism and ameliorates progressive hearing loss in C57BL/6J mice. J Nutr Biochem 26: 1424-1433, 2015.

241. Massafra V, Milona A, Vos HR, Ramos RJJ, Gerrits J, Willemsen ECL, Ramos Pittol JM, Ijssennagger N, Houweling M, Prinsen H, Verhoeven-Duif NM, Burgering BMT, van Mil SWC. Farnesoid X Receptor Activation Promotes Hepatic Amino Acid 
Pajares and Pérez-Sala

Catabolism and Ammonium Clearance in Mice. Gastroenterology 152: 1462-1476 e10, 2017.

242. McDonald I, Connolly M, Tobin AM. A review of psoriasis, a known risk factor for cardiovascular disease and its impact on folate and homocysteine metabolism. $J$ Nutr Metab 2012: 965385, 2012.

243. McGarry DJ, Chakravarty P, Wolf CR, Henderson CJ. Altered protein Sglutathionylation identifies a potential mechanism of resistance to acetaminopheninduced hepatotoxicity. J Pharmacol Exp Ther 355: 137-144, 2015.

244. Mecham JO, Rowitch D, Wallace CD, Stern PH, Hoffman RM. The metabolic defect of methionine dependence occurs frequently in human tumor cell lines. Biochem Biophys Res Commun 117: 429-434, 1983.

245. Medici V, Shibata NM, Kharbanda KK, Islam MS, Keen CL, Kim K, Tillman B, French SW, Halsted CH, LaSalle JM. Maternal choline modifies fetal liver copper, gene expression, DNA methylation, and neonatal growth in the tx-j mouse model of Wilson disease. Epigenetics 9: 286-296, 2014.

246. Medici V, Shibata NM, Kharbanda KK, LaSalle JM, Woods R, Liu S, Engelberg JA, Devaraj S, Torok NJ, Jiang JX, Havel PJ, Lonnerdal B, Kim K, Halsted CH. Wilson's disease: changes in methionine metabolism and inflammation affect global DNA methylation in early liver disease. Hepatology 57: 555-565, 2013.

247. Meister A, Anderson ME. Glutathione. Annu Rev Biochem 52: 711-760, 1983.

248. Michael AJ. Polyamines in Eukaryotes, Bacteria, and Archaea. J Biol Chem 291: 14896-14903, 2016.

249. Milek M, Karas Kuzelicki N, Smid A, Mlinaric-Rascan I. S-adenosylmethionine regulates thiopurine methyltransferase activity and decreases 6-mercaptopurine cytotoxicity in MOLT lymphoblasts. Biochem Pharmacol 77: 1845-1853, 2009. 
Pajares and Pérez-Sala

250. Mindnich RD, Penning TM. Aldo-keto reductase (AKR) superfamily: genomics and annotation. Hum Genomics 3: 362-370, 2009.

251. Mingorance J, Alvarez L, Pajares MA, Mato JM. Recombinant rat liver Sadenosyl-L-methionine synthetase tetramers and dimers are in equilibrium. Int $J$ Biochem Cell Biol 29: 485-491, 1997.

252. Mingorance J, Alvarez L, Sanchez-Gongora E, Mato JM, Pajares MA. Sitedirected mutagenesis of rat liver S-adenosylmethionine synthetase. Identification of a cysteine residue critical for the oligomeric state. Biochem J 315: 761-766, 1996.

253. Minnebo N, Gornemann J, O'Connell N, Van Dessel N, Derua R, Vermunt MW, Page R, Beullens M, Peti W, Van Eynde A, Bollen M. NIPP1 maintains EZH2 phosphorylation and promoter occupancy at proliferation-related target genes. Nucleic Acids Res 41: 842-854, 2013.

254. Mistry RK, Murray TV, Prysyazhna O, Martin D, Burgoyne JR, Santos C, Eaton P, Shah AM, Brewer AC. Transcriptional Regulation of Cystathionine-gamma-Lyase in Endothelial Cells by NADPH Oxidase 4-Dependent Signaling. J Biol Chem 291: 17741788, 2016.

255. Morita SY, Takeuchi A, Kitagawa S. Functional analysis of two isoforms of phosphatidylethanolamine N-methyltransferase. Biochem J 432: 387-398, 2010.

256. Mosharov E, Cranford MR, Banerjee R. The quantitatively important relationship between homocysteine metabolism and glutathione synthesis by the transsulfuration pathway and its regulation by redox changes. Biochemistry 39: 1300513011, 2000.

257. Moskovitz J, Bar-Noy S, Williams WM, Requena J, Berlett BS, Stadtman ER. Methionine sulfoxide reductase (MsrA) is a regulator of antioxidant defense and lifespan in mammals. Proc Natl Acad Sci U S A 98: 12920-12925, 2001. 
Pajares and Pérez-Sala

258. Mudd SH, Ebert MH, Scriver CR. Labile methyl group balances in the human: the role of sarcosine. Metabolism 29: 707-720, 1980.

259. Mudd SH, Levy HL, Tangerman A, Boujet C, Buist N, Davidson-Mundt A, Hudgins L, Oyanagi K, Nagao M, Wilson WG. Isolated persistent hypermethioninemia. Am J Hum Genet 57: 882-892, 1995.

260. Mudd SH, Poole JR. Labile methyl balances for normal humans on various dietary regimens. Metabolism 24: 721-735, 1975.

261. Murray B, Antonyuk SV, Marina A, Van Liempd SM, Lu SC, Mato JM, Hasnain SS, Rojas AL. Structure and function study of the complex that synthesizes Sadenosylmethionine. IUCrJ 1: 240-249, 2014.

262. Nagao M, Tanaka T, Furujo M. Spectrum of mutations associated with methionine adenosyltransferase I/III deficiency among individuals identified during newborn screening in Japan. Mol Genet Metab 110: 460-464, 2013.

263. Neo WH, Lim JF, Grumont R, Gerondakis S, Su IH. c-Rel regulates Ezh2 expression in activated lymphocytes and malignant lymphoid cells. J Biol Chem 289: 31693-31707, 2014.

264. Nieman KM, Rowling MJ, Garrow TA, Schalinske KL. Modulation of methyl group metabolism by streptozotocin-induced diabetes and all-trans-retinoic acid. J Biol Chem 279: 45708-45712, 2004.

265. Niu WN, Yadav PK, Adamec J, Banerjee R. S-glutathionylation enhances human cystathionine beta-synthase activity under oxidative stress conditions. Antioxid Redox Signal 22: 350-361, 2015.

266. O'Leary VB, Ovsepian SV, Carrascosa LG, Buske FA, Radulovic V, Niyazi M, Moertl S, Trau M, Atkinson MJ, Anastasov N. PARTICLE, a Triplex-Forming Long 
Pajares and Pérez-Sala

ncRNA, Regulates Locus-Specific Methylation in Response to Low-Dose Irradiation. Cell Rep 11: 474-485, 2015.

267. Oakley A. Glutathione transferases: a structural perspective. Drug Metab Rev 43: 138-151, 2011.

268. Ogawa H, Gomi T, Takata Y, Date T, Fujioka M. Recombinant expression of rat glycine N-methyltransferase and evidence for contribution of N-terminal acetylation to co-operative binding of S-adenosylmethionine. Biochem J 327: 407-412, 1997.

269. Ogawa H, Ishiguro Y, Fujioka M. Guanidoacetate methyltransferase from rat liver: purification, properties, and evidence for the involvement of sulfhydryl groups for activity. Arch Biochem Biophys 226: 265-275, 1983.

270. Okano M, Bell DW, Haber DA, Li E. DNA methyltransferases Dnmt3a and Dnmt3b are essential for de novo methylation and mammalian development. Cell 99: 247-257, 1999.

271. Orentreich N, Matias JR, DeFelice A, Zimmerman JA. Low methionine ingestion by rats extends life span. J Nutr 123: 269-274, 1993.

272. Ou X, Yang H, Ramani K, Ara AI, Chen H, Mato JM, Lu SC. Inhibition of human betaine-homocysteine methyltransferase expression by S-adenosylmethionine and methylthioadenosine. Biochem J 401: 87-96, 2007.

273. Pajares MA, Alemany S, Varela I, Marin Cao D, Mato JM. Purification and photoaffinity labelling of lipid methyltransferase from rat liver. Biochem $J$ 223: 61-66, 1984.

274. Pajares MA, Corrales FJ, Ochoa P, Mato JM. The role of cysteine-150 in the structure and activity of rat liver S-adenosyl-L-methionine synthetase. Biochem $J 274$ : 225-229, 1991. 
Pajares and Pérez-Sala

275. Pajares MA, Duran C, Corrales F, Mato JM. Protein kinase C phosphorylation of rat liver S-adenosylmethionine synthetase: dissociation and production of an active monomer. Biochem J 303: 949-955, 1994.

276. Pajares MA, Duran C, Corrales F, Pliego MM, Mato JM. Modulation of rat liver S-adenosylmethionine synthetase activity by glutathione. J Biol Chem 267: 1759817605, 1992.

277. Pajares MA, Markham GD. Methionine adenosyltransferase (sadenosylmethionine synthetase). Adv Enzymol Relat Areas Mol Biol 78: 449-521, 2011. 278. Pajares MA, Villalba M, Mato JM. Purification of phospholipid methyltransferase from rat liver microsomal fraction. Biochem J 237: 699-705, 1986.

279. Palmer JL, Abeles RH. The mechanism of action of S-adenosylhomocysteinase. J Biol Chem 254: 1217-1226, 1979.

280. Parkhitko AA, Binari R, Zhang N, Asara JM, Demontis F, Perrimon N. Tissuespecific down-regulation of S-adenosyl-homocysteine via suppression of dAhcyL1/dAhcyL2 extends health span and life span in Drosophila. Genes Dev 30: 1409-1422, 2016.

281. Pasini D, Bracken AP, Jensen MR, Lazzerini Denchi E, Helin K. Suz12 is essential for mouse development and for EZH2 histone methyltransferase activity. EMBO J 23: 4061-4071, 2004.

282. Paul BD, Sbodio JI, Xu R, Vandiver MS, Cha JY, Snowman AM, Snyder SH. Cystathionine gamma-lyase deficiency mediates neurodegeneration in Huntington's disease. Nature 509: 96-100, 2014.

283. Pauwels S, Ghosh M, Duca RC, Bekaert B, Freson K, Huybrechts I, Langie SA, Koppen G, Devlieger R, Godderis L. Maternal intake of methyl-group donors affects DNA methylation of metabolic genes in infants. Clin Epigenetics 9: 16, 2017. 
Pajares and Pérez-Sala

284. Pavlova NN, Thompson CB. The Emerging Hallmarks of Cancer Metabolism. Cell Metab 23: 27-47, 2016.

285. Paz MF, Fraga MF, Avila S, Guo M, Pollan M, Herman JG, Esteller M. A systematic profile of DNA methylation in human cancer cell lines. Cancer Res 63: 1114-1121, 2003.

286. Pelech SL, Power E, Vance DE. Activities of the phosphatidylcholine biosynthetic enzymes in rat liver during development. Can J Biochem Cell Biol 61: 1147-1152, 1983.

287. Pendleton KE, Chen B, Liu K, Hunter OV, Xie Y, Tu BP, Conrad NK. The U6 snRNA m6A Methyltransferase METTL16 Regulates SAM Synthetase Intron Retention. Cell 169: 824-835 e14, 2017.

288. Peng H, Dara L, Li TW, Zheng Y, Yang H, Tomasi ML, Tomasi I, Giordano P, Mato JM, Lu SC. MAT2B-GIT1 interplay activates MEK1/ERK 1 and 2 to induce growth in human liver and colon cancer. Hepatology 57: 2299-2313, 2013.

289. Peng H, Li TW, Yang H, Moyer MP, Mato JM, Lu SC. Methionine adenosyltransferase 2B-GIT1 complex serves as a scaffold to regulate Ras/Raf/MEK1/2 activity in human liver and colon cancer cells. Am J Pathol 185: 1135-1144, 2015.

290. Peng L, Yuan Z, Ling H, Fukasawa K, Robertson K, Olashaw N, Koomen J, Chen J, Lane WS, Seto E. SIRT1 deacetylates the DNA methyltransferase 1 (DNMT1) protein and alters its activities. Mol Cell Biol 31: 4720-4734, 2011.

291. Perez C, Perez-Zuniga FJ, Garrido F, Reytor E, Portillo F, Pajares MA. The Oncogene PDRG1 Is an Interaction Target of Methionine Adenosyltransferases. PLoS One 11: e0161672, 2016. 
Pajares and Pérez-Sala

292. Perez-Mato I, Castro C, Ruiz FA, Corrales FJ, Mato JM. Methionine adenosyltransferase S-nitrosylation is regulated by the basic and acidic amino acids surrounding the target thiol. J Biol Chem 274: 17075-17079, 1999.

293. Perez-Miguelsanz J, Vallecillo N, Garrido F, Reytor E, Perez-Sala D, Pajares MA. Betaine homocysteine S-methyltransferase emerges as a new player of the nuclear methionine cycle. Biochim Biophys Acta 1864: 1165-1182, 2017.

294. Perrone CE, Mattocks DA, Plummer JD, Chittur SV, Mohney R, Vignola K, Orentreich DS, Orentreich N. Genomic and metabolic responses to methioninerestricted and methionine-restricted, cysteine-supplemented diets in Fischer 344 rat inguinal adipose tissue, liver and quadriceps muscle. $J$ Nutrigenet Nutrigenomics 5: 132-157, 2012.

295. Petrash JM. All in the family: aldose reductase and closely related aldo-keto reductases. Cell Mol Life Sci 61: 737-749, 2004.

296. Phulukdaree A, Moodley D, Khan S, Chuturgoon AA. Atorvastatin increases miR-124a expression: a mechanism of Gamt modulation in liver cells. $J$ Cell Biochem 116: 2620-2627, 2015.

297. Piper M, Barry G, Harvey TJ, McLeay R, Smith AG, Harris L, Mason S, Stringer BW, Day BW, Wray NR, Gronostajski RM, Bailey TL, Boyd AW, Richards LJ. NFIB-mediated repression of the epigenetic factor Ezh2 regulates cortical development. J Neurosci 34: 2921-2930, 2014.

298. Pradhan S, Chin HG, Esteve PO, Jacobsen SE. SET7/9 mediated methylation of non-histone proteins in mammalian cells. Epigenetics 4: 383-387, 2009.

299. Qin W, Leonhardt H, Spada F. Usp7 and Uhrf1 control ubiquitination and stability of the maintenance DNA methyltransferase Dnmt1. J Cell Biochem 112: 439$444,2011$. 
Pajares and Pérez-Sala

300. Qin W, Wolf P, Liu N, Link S, Smets M, La Mastra F, Forne I, Pichler G, Horl D, Fellinger K, Spada F, Bonapace IM, Imhof A, Harz H, Leonhardt H. DNA methylation requires a DNMT1 ubiquitin interacting motif (UIM) and histone ubiquitination. Cell Res 25: 911-929, 2015.

301. Qiu Y, Benet LZ, Burlingame AL. Identification of the hepatic protein targets of reactive metabolites of acetaminophen in vivo in mice using two-dimensional gel electrophoresis and mass spectrometry. J Biol Chem 273: 17940-17953, 1998.

302. Quemeneur L, Gerland LM, Flacher M, Ffrench M, Revillard JP, Genestier L. Differential control of cell cycle, proliferation, and survival of primary $\mathrm{T}$ lymphocytes by purine and pyrimidine nucleotides. J Immunol 170: 4986-4995, 2003.

303. Quinn AM, Harvey RG, Penning TM. Oxidation of PAH trans-dihydrodiols by human aldo-keto reductase AKR1B10. Chem Res Toxicol 21: 2207-2215, 2008.

304. Radomski N, Kaufmann C, Dreyer C. Nuclear accumulation of Sadenosylhomocysteine hydrolase in transcriptionally active cells during development of Xenopus laevis. Mol Biol Cell 10: 4283-4298, 1999.

305. Ramani K, Donoyan S, Tomasi ML, Park S. Role of methionine adenosyltransferase alpha2 and beta phosphorylation and stabilization in human hepatic stellate cell trans-differentiation. J Cell Physiol 230: 1075-1085, 2015.

306. Ratnam S, Maclean KN, Jacobs RL, Brosnan ME, Kraus JP, Brosnan JT. Hormonal regulation of cystathionine beta-synthase expression in liver. J Biol Chem 277: 42912-42918, 2002.

307. Renga B, Cipriani S, Carino A, Simonetti M, Zampella A, Fiorucci S. Reversal of Endothelial Dysfunction by GPBAR1 Agonism in Portal Hypertension Involves a AKT/FOXOA1 Dependent Regulation of H2S Generation and Endothelin-1. PLoS One 10: e0141082, 2015. 
Pajares and Pérez-Sala

308. Renga B, Mencarelli A, Migliorati M, Distrutti E, Fiorucci S. Bile-acid-activated farnesoid $\mathrm{X}$ receptor regulates hydrogen sulfide production and hepatic microcirculation. World J Gastroenterol 15: 2097-2108, 2009.

309. Resseguie M, Song J, Niculescu MD, da Costa KA, Randall TA, Zeisel SH. Phosphatidylethanolamine N-methyltransferase (PEMT) gene expression is induced by estrogen in human and mouse primary hepatocytes. FASEB J 21: 2622-2632, 2007.

310. Reuter S, Gupta SC, Chaturvedi MM, Aggarwal BB. Oxidative stress, inflammation, and cancer: how are they linked? Free Radic Biol Med 49: 1603-1616, 2010.

311. Reytor E, Perez-Miguelsanz J, Alvarez L, Perez-Sala D, Pajares MA. Conformational signals in the C-terminal domain of methionine adenosyltransferase I/III determine its nucleocytoplasmic distribution. FASEB J 23: 3347-3360, 2009.

312. Richie JP, Jr., Leutzinger Y, Parthasarathy S, Malloy V, Orentreich N, Zimmerman JA. Methionine restriction increases blood glutathione and longevity in F344 rats. FASEB J 8: 1302-1307, 1994.

313. Rigbolt KT, Prokhorova TA, Akimov V, Henningsen J, Johansen PT, Kratchmarova I, Kassem M, Mann M, Olsen JV, Blagoev B. System-wide temporal characterization of the proteome and phosphoproteome of human embryonic stem cell differentiation. Sci Signal 4: rs3, 2011.

314. Rios-Avila L, Coats B, Ralat M, Chi YY, Midttun O, Ueland PM, Stacpoole PW, Gregory JF, 3rd. Pyridoxine supplementation does not alter in vivo kinetics of onecarbon metabolism but modifies patterns of one-carbon and tryptophan metabolites in vitamin B-6-insufficient oral contraceptive users. Am J Clin Nutr 102: 616-625, 2015.

315. Robertson KD, Uzvolgyi E, Liang G, Talmadge C, Sumegi J, Gonzales FA, Jones PA. The human DNA methyltransferases (DNMTs) 1, 3a and 3b: coordinate 
Pajares and Pérez-Sala

mRNA expression in normal tissues and overexpression in tumors. Nucleic Acids Res 27: 2291-2298, 1999.

316. Robertson KD, Wolffe AP. DNA methylation in health and disease. Nat Rev Genet 1: 11-19, 2000.

317. Rodriguez JL, Boukaba A, Sandoval J, Georgieva EI, Latasa MU, GarciaTrevijano ER, Serviddio G, Nakamura T, Avila MA, Sastre J, Torres L, Mato JM, Lopez-Rodas G. Transcription of the MAT2A gene, coding for methionine adenosyltransferase, is up-regulated by $\mathrm{E} 2 \mathrm{~F}$ and $\mathrm{Sp} 1$ at a chromatin level during proliferation of liver cells. Int J Biochem Cell Biol 39: 842-850, 2007.

318. Rosas-Rodriguez JA, Valenzuela-Soto EM. Inactivation of porcine kidney betaine aldehyde dehydrogenase by hydrogen peroxide. Chem Biol Interact 191: 159$164,2011$.

319. Rowling MJ, Schalinske KL. Retinoic acid and glucocorticoid treatment induce hepatic glycine N-methyltransferase and lower plasma homocysteine concentrations in rats and rat hepatoma cells. $J$ Nutr 133: 3392-3398, 2003.

320. Sahasrabuddhe AA, Chen X, Chung F, Velusamy T, Lim MS, ElenitobaJohnson KS. Oncogenic Y641 mutations in EZH2 prevent Jak2/beta-TrCP-mediated degradation. Oncogene 34: 445-454, 2015.

321. Sakai M, Muramatsu M. Regulation of glutathione transferase P: a tumor marker of hepatocarcinogenesis. Biochem Biophys Res Commun 357: 575-578, 2007.

322. Sanchez-Gomez FJ, Diez-Dacal B, Pajares MA, Llorca O, Perez-Sala D. Cyclopentenone prostaglandins with dienone structure promote cross-linking of the chemoresistance-inducing enzyme glutathione transferase P1-1. Mol Pharmacol 78: 723-733, 2010. 
Pajares and Pérez-Sala

323. Sanchez-Gomez FJ, Dorado CG, Ayuso P, Agundez JA, Pajares MA, Perez-Sala D. Modulation of GSTP1-1 oligomerization by electrophilic inflammatory mediators and reactive drugs. Inflamm Allergy Drug Targets 12: 162-171, 2013.

324. Sanchez-Gongora E, Pastorino JG, Alvarez L, Pajares MA, Garcia C, Vina JR, Mato JM, Farber JL. Increased sensitivity to oxidative injury in chinese hamster ovary cells stably transfected with rat liver S-adenosylmethionine synthetase cDNA. Biochem J319: 767-773, 1996.

325. Sanchez-Perez GF, Gasset M, Calvete JJ, Pajares MA. Role of an intrasubunit disulfide in the association state of the cytosolic homo-oligomer methionine adenosyltransferase. J Biol Chem 278: 7285-7293, 2003.

326. Sanchez-Roman I, Gomez A, Perez I, Sanchez C, Suarez H, Naudi A, Jove M, Lopez-Torres M, Pamplona R, Barja G. Effects of aging and methionine restriction applied at old age on ROS generation and oxidative damage in rat liver mitochondria. Biogerontology 13: 399-411, 2012.

327. Sanz A, Caro P, Ayala V, Portero-Otin M, Pamplona R, Barja G. Methionine restriction decreases mitochondrial oxygen radical generation and leak as well as oxidative damage to mitochondrial DNA and proteins. FASEB J 20: 1064-1073, 2006.

328. Sarma K, Margueron R, Ivanov A, Pirrotta V, Reinberg D. Ezh2 requires PHF1 to efficiently catalyze H3 lysine 27 trimethylation in vivo. Mol Cell Biol 28: 2718-2731, 2008.

329. Satow R, Shitashige M, Kanai Y, Takeshita F, Ojima H, Jigami T, Honda K, Kosuge T, Ochiya T, Hirohashi S, Yamada T. Combined functional genome survey of therapeutic targets for hepatocellular carcinoma. Clin Cancer Res 16: 2518-2528, 2010. 
Pajares and Pérez-Sala

330. Schnackenberg LK, Chen M, Sun J, Holland RD, Dragan Y, Tong W, Welsh W, Beger RD. Evaluations of the trans-sulfuration pathway in multiple liver toxicity studies. Toxicol Appl Pharmacol 235: 25-32, 2009.

331. Seinen ML, de Boer NK, Smid K, van Asseldonk DP, Bouma G, van Bodegraven AA, Peters GJ. Allopurinol enhances the activity of hypoxanthine-guanine phosphoribosyltransferase in inflammatory bowel disease patients during low-dose thiopurine therapy: preliminary data of an ongoing series. Nucleosides Nucleotides Nucleic Acids 30: 1085-1090, 2011.

332. Sen N, Paul BD, Gadalla MM, Mustafa AK, Sen T, Xu R, Kim S, Snyder SH. Hydrogen sulfide-linked sulfhydration of NF-kappaB mediates its antiapoptotic actions. Mol Cell 45: 13-24, 2012.

333. Sewalt RG, van der Vlag J, Gunster MJ, Hamer KM, den Blaauwen JL, Satijn DP, Hendrix T, van Driel R, Otte AP. Characterization of interactions between the mammalian polycomb-group proteins Enx1/EZH2 and EED suggests the existence of different mammalian polycomb-group protein complexes. Mol Cell Biol 18: 3586-3595, 1998.

334. Shafqat N, Muniz JR, Pilka ES, Papagrigoriou E, von Delft F, Oppermann U, Yue WW. Insight into S-adenosylmethionine biosynthesis from the crystal structures of the human methionine adenosyltransferase catalytic and regulatory subunits. Biochem $J$ 452: 27-36, 2013.

335. Sharma A, Bartell SM, Baile CA, Chen B, Podolsky RH, McIndoe RA, She JX. Hepatic gene expression profiling reveals key pathways involved in leptin-mediated weight loss in ob/ob mice. PLoS One 5: e12147, 2010. 
Pajares and Pérez-Sala

336. Shen Y, Shen Z, Miao L, Xin X, Lin S, Zhu Y, Guo W, Zhu YZ. miRNA-30 family inhibition protects against cardiac ischemic injury by regulating cystathioninegamma-lyase expression. Antioxid Redox Signal 22: 224-240, 2015.

337. Shields DJ, Agellon LB, Vance DE. Structure, expression profile and alternative processing of the human phosphatidylethanolamine N-methyltransferase (PEMT) gene. Biochim Biophys Acta 1532: 105-114, 2001.

338. Shields DJ, Altarejos JY, Wang X, Agellon LB, Vance DE. Molecular dissection of the S-adenosylmethionine-binding site of phosphatidylethanolamine Nmethyltransferase. J Biol Chem 278: 35826-35836, 2003.

339. Shimizu K, Abe M, Yokoyama S, Takahashi H, Sawada N, Mori M, Tsukada K. Decreased activities of S-adenosylmethionine synthetase isozymes in hereditary hepatitis in Long-Evans rats. Life Sci 46: 1837-1842, 1990.

340. Slomka M, Zieminska E, Lazarewicz J. Nicotinamide and 1-methylnicotinamide reduce homocysteine neurotoxicity in primary cultures of rat cerebellar granule cells. Acta Neurobiol Exp (Wars) 68: 1-9, 2008.

341. Snykers S, Henkens T, De Rop E, Vinken M, Fraczek J, De Kock J, De Prins E, Geerts A, Rogiers V, Vanhaecke T. Role of epigenetics in liver-specific gene transcription, hepatocyte differentiation and stem cell reprogrammation. $J$ Hepatol 51: 187-211, 2009.

342. Someya S, Yu W, Hallows WC, Xu J, Vann JM, Leeuwenburgh C, Tanokura M, Denu JM, Prolla TA. Sirt3 mediates reduction of oxidative damage and prevention of age-related hearing loss under caloric restriction. Cell 143: 802-812, 2010.

343. Song J, da Costa KA, Fischer LM, Kohlmeier M, Kwock L, Wang S, Zeisel SH. Polymorphism of the PEMT gene and susceptibility to nonalcoholic fatty liver disease (NAFLD). FASEB J 19: 1266-1271, 2005. 
Pajares and Pérez-Sala

344. Song J, Du Z, Ravasz M, Dong B, Wang Z, Ewing RM. A Protein Interaction between beta-Catenin and Dnmt1 Regulates Wnt Signaling and DNA Methylation in Colorectal Cancer Cells. Mol Cancer Res 13: 969-981, 2015.

345. Srivastava SK, Ramana KV, Bhatnagar A. Role of aldose reductase and oxidative damage in diabetes and the consequent potential for therapeutic options. Endocr Rev 26: 380-392, 2005.

346. Srivastava SK, Ramana KV, Chandra D, Srivastava S, Bhatnagar A. Regulation of aldose reductase and the polyol pathway activity by nitric oxide. Chem Biol Interact 143-144: 333-340, 2003.

347. Steegborn C, Clausen T, Sondermann P, Jacob U, Worbs M, Marinkovic S, Huber R, Wahl MC. Kinetics and inhibition of recombinant human cystathionine gamma-lyase. Toward the rational control of transsulfuration. J Biol Chem 274: 1267512684, 1999.

348. Stender S, Chakrabarti RS, Xing C, Gotway G, Cohen JC, Hobbs HH. Adultonset liver disease and hepatocellular carcinoma in S-adenosylhomocysteine hydrolase deficiency. Mol Genet Metab 116: 269-274, 2015.

349. Sterling KM. 4S polycyclic aromatic hydrocarbon receptor (glycine Nmethyltransferase) and the aryl hydrocarbon receptor nuclear translocator (hypoxia inducible factor-1beta) interaction in Chinese hamster ovary and rat hepatoma cells: $4 \mathrm{~S}$ PAH-R/ARNT hetero-oligomers? J Cell Biochem 112: 2015-2018, 2011.

350. Straus DS, Pascual G, Li M, Welch JS, Ricote M, Hsiang CH, Sengchanthalangsy LL, Ghosh G, Glass CK. 15-deoxy-delta 12,14-prostaglandin J2 inhibits multiple steps in the NF-kappa B signaling pathway. Proc Natl Acad Sci U S A 97: 4844-4849, 2000. 
Pajares and Pérez-Sala

351. Sufrin JR, Finckbeiner S, Oliver CM. Marine-derived metabolites of Sadenosylmethionine as templates for new anti-infectives. Mar Drugs 7: 401-434, 2009.

352. Sugimura T, Birnbaum SM, Winitz M, Greenstein JP. Quantitative nutritional studies with water-soluble, chemically defined diets. VIII. The forced feeding of diets each lacking in one essential amino acid. Arch Biochem Biophys 81: 448-455, 1959.

353. Sun Q, Collins R, Huang S, Holmberg-Schiavone L, Anand GS, Tan CH, vanden-Berg S, Deng LW, Moore PK, Karlberg T, Sivaraman J. Structural basis for the inhibition mechanism of human cystathionine gamma-lyase, an enzyme responsible for the production of H(2)S. J Biol Chem 284: 3076-3085, 2009.

354. Surtees R, Leonard J, Austin S. Association of demyelination with deficiency of cerebrospinal-fluid S-adenosylmethionine in inborn errors of methyl-transfer pathway. Lancet 338: 1550-1554, 1991.

355. Sutter BM, Wu X, Laxman S, Tu BP. Methionine inhibits autophagy and promotes growth by inducing the SAM-responsive methylation of PP2A. Cell 154: 403$415,2013$.

356. Szegedi SS, Castro CC, Koutmos M, Garrow TA. Betaine-homocysteine Smethyltransferase-2 is an S-methylmethionine-homocysteine methyltransferase. J Biol Chem 283: 8939-8945, 2008.

357. Talaei F, van Praag VM, Henning RH. Hydrogen sulfide restores a normal morphological phenotype in Werner syndrome fibroblasts, attenuates oxidative damage and modulates mTOR pathway. Pharmacol Res 74: 34-44, 2013.

358. Tang D, Kang R, Livesey KM, Cheh CW, Farkas A, Loughran P, Hoppe G, Bianchi ME, Tracey KJ, Zeh HJ, 3rd, Lotze MT. Endogenous HMGB1 regulates autophagy. J Cell Biol 190: 881-892, 2010. 
Pajares and Pérez-Sala

359. Tang X, Milyavsky M, Shats I, Erez N, Goldfinger N, Rotter V. Activated p53 suppresses the histone methyltransferase EZH2 gene. Oncogene 23: 5759-5769, 2004.

360. Taoka S, Lepore BW, Kabil O, Ojha S, Ringe D, Banerjee R. Human cystathionine beta-synthase is a heme sensor protein. Evidence that the redox sensor is heme and not the vicinal cysteines in the CXXC motif seen in the crystal structure of the truncated enzyme. Biochemistry 41: 10454-10461, 2002.

361. Taylor RT, Hanna LM. Folate-dependent enzymes in cultured Chinese hamster ovary cells: induction of 5-methyltetrahydrofolate homocysteine cobalamin methyltransferase by folate and methionine. Arch Biochem Biophys 171: 507-520, 1975. 362. Tew KD, Townsend DM. Glutathione-s-transferases as determinants of cell survival and death. Antioxid Redox Signal 17: 1728-1737, 2012.

363. Tomasi ML, Ryoo M, Ramani K, Tomasi I, Giordano P, Mato JM, Lu SC. Methionine adenosyltransferase alpha2 sumoylation positively regulate Bcl-2 expression in human colon and liver cancer cells. Oncotarget 6: 37706-37723, 2015.

364. Tomasi ML, Ryoo M, Skay A, Tomasi I, Giordano P, Mato JM, Lu SC. Polyamine and methionine adenosyltransferase $2 \mathrm{~A}$ crosstalk in human colon and liver cancer. Exp Cell Res 319: 1902-1911, 2013.

365. Tomasi ML, Tomasi I, Ramani K, Pascale RM, Xu J, Giordano P, Mato JM, Lu SC. S-adenosyl methionine regulates ubiquitin-conjugating enzyme 9 protein expression and sumoylation in murine liver and human cancers. Hepatology 56: 982993, 2012.

366. Torre L, Lopez-Rodas G, Latasa MU, Carretero MV, Boukaba A, Rodriguez JL, Franco L, Mato JM, Avila MA. DNA methylation and histone acetylation of rat methionine adenosyltransferase 1A and 2A genes is tissue-specific. Int J Biochem Cell Biol 32: 397-404, 2000. 
Pajares and Pérez-Sala

367. Torres L, Avila MA, Carretero MV, Latasa MU, Caballeria J, Lopez-Rodas G, Boukaba A, Lu SC, Franco L, Mato JM. Liver-specific methionine adenosyltransferase MAT1A gene expression is associated with a specific pattern of promoter methylation and histone acetylation: implications for MAT1A silencing during transformation. FASEB J 14: 95-102, 2000.

368. Tsukada K, Okada G. S-Adenosylmethionine synthetase isozyme patterns from rat hepatoma induced by N-2-fluorenylacetamide. Biochem Biophys Res Commun 94: 1078-1082, 1980.

369. Tsuzura H, Genda T, Sato S, Murata A, Kanemitsu Y, Narita Y, Ishikawa S, Kikuchi T, Mori M, Hirano K, Iijima K, Wada R, Ichida T. Expression of aldo-keto reductase family 1 member b10 in the early stages of human hepatocarcinogenesis. Int $J$ Mol Sci 15: 6556-6568, 2014.

370. Turner MA, Yang X, Yin D, Kuczera K, Borchardt RT, Howell PL. Structure and function of S-adenosylhomocysteine hydrolase. Cell Biochem Biophys 33: 101-125, 2000.

371. Turner MA, Yuan CS, Borchardt RT, Hershfield MS, Smith GD, Howell PL. Structure determination of selenomethionyl S-adenosylhomocysteine hydrolase using data at a single wavelength. Nat Struct Biol 5: 369-376, 1998.

372. Ueland PM, Refsum H. Plasma homocysteine, a risk factor for vascular disease: plasma levels in health, disease, and drug therapy. J Lab Clin Med 114: 473-501, 1989.

373. Ugrinova I, Pasheva E. HMGB1 Protein: A Therapeutic Target Inside and Outside the Cell. Adv Protein Chem Struct Biol 107: 37-76, 2017.

374. Ulanovskaya OA, Zuhl AM, Cravatt BF. NNMT promotes epigenetic remodeling in cancer by creating a metabolic methylation sink. Nat Chem Biol 9: 300306, 2013. 
Pajares and Pérez-Sala

375. Vailati-Riboni M, Osorio JS, Trevisi E, Luchini D, Loor JJ. Supplemental Smartamine $\mathrm{M}$ in higher-energy diets during the prepartal period improves hepatic biomarkers of health and oxidative status in Holstein cows. J Anim Sci Biotechnol 8: 17, 2017.

376. Vance DE. Physiological roles of phosphatidylethanolamine Nmethyltransferase. Biochim Biophys Acta 1831: 626-632, 2013.

377. Vazquez-Chantada M, Fernandez-Ramos D, Embade N, Martinez-Lopez N, Varela-Rey M, Woodhoo A, Luka Z, Wagner C, Anglim PP, Finnell RH, Caballeria J, Laird-Offringa IA, Gorospe M, Lu SC, Mato JM, Martinez-Chantar ML. HuR/MethylHuR and AU-Rich RNA Binding Factor 1 Regulate the Methionine Adenosyltransferase Expressed During Liver Proliferation, Differentiation, and Carcinogenesis. Gastroenterology 138: 1943-1953, 2010.

378. Vazquez-Chantada M, Fernandez-Ramos D, Embade N, Martinez-Lopez N, Varela-Rey M, Woodhoo A, Luka Z, Wagner C, Anglim PP, Finnell RH, Caballeria J, Laird-Offringa IA, Gorospe M, Lu SC, Mato JM, Martinez-Chantar ML. HuR/methylHuR and AUF1 regulate the MAT expressed during liver proliferation, differentiation, and carcinogenesis. Gastroenterology 138: 1943-1953, 2010.

379. Vicente JB, Colaco HG, Sarti P, Leandro P, Giuffre A. S-Adenosyl-1-methionine Modulates $\mathrm{CO}$ and $\mathrm{NO}^{*}$ Binding to the Human H2S-generating Enzyme Cystathionine beta-Synthase. J Biol Chem 291: 572-581, 2016.

380. Villalba M, Pajares MA, Renart MF, Mato JM. Protein kinase C catalyses the phosphorylation and activation of rat liver phospholipid methyltransferase. Biochem $J$ 241: 911-916, 1987.

381. Vire E, Brenner C, Deplus R, Blanchon L, Fraga M, Didelot C, Morey L, Van Eynde A, Bernard D, Vanderwinden JM, Bollen M, Esteller M, Di Croce L, de Launoit 
Pajares and Pérez-Sala

Y, Fuks F. The Polycomb group protein EZH2 directly controls DNA methylation. Nature 439: 871-874, 2006.

382. Vitvitsky V, Dayal S, Stabler S, Zhou Y, Wang H, Lentz SR, Banerjee R. Perturbations in homocysteine-linked redox homeostasis in a murine model for hyperhomocysteinemia. Am J Physiol Regul Integr Comp Physiol 287: R39-46, 2004.

383. Vugrek O, Beluzic R, Nakic N, Mudd SH. S-adenosylhomocysteine hydrolase (AHCY) deficiency: two novel mutations with lethal outcome. Hum Mutat 30: E555$565,2009$.

384. Wagner C, Briggs WT, Cook RJ. Inhibition of glycine N-methyltransferase activity by folate derivatives: implications for regulation of methyl group metabolism. Biochem Biophys Res Commun 127: 746-752, 1985.

385. Wagner C, Decha-Umphai W, Corbin J. Phosphorylation modulates the activity of glycine N-methyltransferase, a folate binding protein. In vitro phosphorylation is inhibited by the natural folate ligand. J Biol Chem 264: 9638-9642, 1989.

386. Walsh CT, Garneau-Tsodikova S, Gatto GJ, Jr. Protein posttranslational modifications: the chemistry of proteome diversifications. Angew Chem Int Ed Engl 44: 7342-7372, 2005.

387. Wan C, Borgeson B, Phanse S, Tu F, Drew K, Clark G, Xiong X, Kagan O, Kwan J, Bezginov A, Chessman K, Pal S, Cromar G, Papoulas O, Ni Z, Boutz DR, Stoilova S, Havugimana PC, Guo X, Malty RH, Sarov M, Greenblatt J, Babu M, Derry WB, Tillier ER, Wallingford JB, Parkinson J, Marcotte EM, Emili A. Panorama of ancient metazoan macromolecular complexes. Nature 525: 339-344, 2015.

388. Wan J, Zhan J, Li S, Ma J, Xu W, Liu C, Xue X, Xie Y, Fang W, Chin YE, Zhang H. PCAF-primed EZH2 acetylation regulates its stability and promotes lung adenocarcinoma progression. Nucleic Acids Res 43: 3591-3604, 2015. 
Pajares and Pérez-Sala

389. Wang C, Yan R, Luo D, Watabe K, Liao DF, Cao D. Aldo-keto reductase family 1 member B10 promotes cell survival by regulating lipid synthesis and eliminating carbonyls. J Biol Chem 284: 26742-26748, 2009.

390. Wang J, Zhang X, Wang L, Yang Y, Dong Z, Wang H, Du L, Wang C. MicroRNA-214 suppresses oncogenesis and exerts impact on prognosis by targeting PDRG1 in bladder cancer. PLoS One 10: e0118086, 2015.

391. Wang L, Tang ZP, Zhao W, Cong BH, Lu JQ, Tang XL, Li XH, Zhu XY, Ni X. MiR-22/Sp-1 Links Estrogens With the Up-Regulation of Cystathionine gamma-Lyase in Myocardium, Which Contributes to Estrogenic Cardioprotection Against Oxidative Stress. Endocrinology 156: 2124-2137, 2015.

392. Wang M, Guo Z, Wang S. The binding site for the transcription factor, NFkappaB, on the cystathionine gamma-lyase promoter is critical for LPS-induced cystathionine gamma-lyase expression. Int J Mol Med 34: 639-645, 2014.

393. Wang R. Physiological implications of hydrogen sulfide: a whiff exploration that blossomed. Physiol Rev 92: 791-896, 2012.

394. Wang YC, Tang FY, Chen SY, Chen YM, Chiang EP. Glycine-N methyltransferase expression in HepG2 cells is involved in methyl group homeostasis by regulating transmethylation kinetics and DNA methylation. $J$ Nutr 141: 777-782, 2011.

395. Womack M, Kemmerer KS, Rose WC. The relation of dietary methionine and cysteine to growth. J Biol Chem 121: 403-410, 1937.

396. Wu SM, Huang YH, Lu YH, Chien LF, Yeh CT, Tsai MM, Liao CH, Chen WJ, Liao CJ, Cheng WL, Lin KH. Thyroid hormone receptor-mediated regulation of the methionine adenosyltransferase 1 gene is associated with cell invasion in hepatoma cell lines. Cell Mol Life Sci 67: 1831-1843, 2010. 
Pajares and Pérez-Sala

397. Xia H, Ooi LL, Hui KM. MiR-214 targets beta-catenin pathway to suppress invasion, stem-like traits and recurrence of human hepatocellular carcinoma. PLoS One 7: e44206, 2012.

398. Xia M, Chen Y, Wang LC, Zandi E, Yang H, Bemanian S, Martinez-Chantar ML, Mato JM, Lu SC. Novel function and intracellular localization of methionine adenosyltransferase 2 \{beta\} splicing variants. J Biol Chem 285: 20015-20021, 2010.

399. Xiao B, Sanders MJ, Carmena D, Bright NJ, Haire LF, Underwood E, Patel BR, Heath RB, Walker PA, Hallen S, Giordanetto F, Martin SR, Carling D, Gamblin SJ. Structural basis of AMPK regulation by small molecule activators. Nat Commun 4: 3017, 2013.

400. $\mathrm{Xu} \mathrm{L}$, Chen J, Gao J, Yu H, Yang P. Crosstalk of homocysteinylation, methylation and acetylation on histone H3. Analyst 140: 3057-3063, 2015.

401. Yan J, Li B, Lin B, Lee PT, Chung TH, Tan J, Bi C, Lee XT, Selvarajan V, Ng SB, Yang H, Yu Q, Chng WJ. EZH2 phosphorylation by JAK3 mediates a switch to noncanonical function in natural killer/T-cell lymphoma. Blood 128: 948-958, 2016.

402. Yang F, Zhang L, Huo XS, Yuan JH, Xu D, Yuan SX, Zhu N, Zhou WP, Yang GS, Wang YZ, Shang JL, Gao CF, Zhang FR, Wang F, Sun SH. Long noncoding RNA high expression in hepatocellular carcinoma facilitates tumor growth through enhancer of zeste homolog 2 in humans. Hepatology 54: 1679-1689, 2011.

403. Yang G, Pei Y, Teng H, Cao Q, Wang R. Specificity protein-1 as a critical regulator of human cystathionine gamma-lyase in smooth muscle cells. J Biol Chem 286: 26450-26460, 2011.

404. Yang H, Ara AI, Magilnick N, Xia M, Ramani K, Chen H, Lee TD, Mato JM, Lu SC. Expression pattern, regulation, and functions of methionine adenosyltransferase 2beta splicing variants in hepatoma cells. Gastroenterology 134: 281-291, 2008. 
Pajares and Pérez-Sala

405. Yang H, Cho ME, Li TW, Peng H, Ko KS, Mato JM, Lu SC. MicroRNAs regulate methionine adenosyltransferase 1A expression in hepatocellular carcinoma. $J$ Clin Invest 123: 285-298, 2013.

406. Yang H, Huang ZZ, Wang J, Lu SC. The role of c-Myb and Sp1 in the upregulation of methionine adenosyltransferase $2 \mathrm{~A}$ gene expression in human hepatocellular carcinoma. FASEB J 15: 1507-1516, 2001.

407. Yang H, Li TW, Peng J, Mato JM, Lu SC. Insulin-like growth factor 1 activates methionine adenosyltransferase 2A transcription by multiple pathways in human colon cancer cells. Biochem J 436: 507-516, 2011.

408. Yang H, Liu T, Wang J, Li TW, Fan W, Peng H, Krishnan A, Gores GJ, Mato JM, Lu SC. Deregulated methionine adenosyltransferase alpha1, c-Myc, and Maf proteins together promote cholangiocarcinoma growth in mice and humans(double dagger). Hepatology 64: 439-455, 2016.

409. Yang H, Sadda MR, Yu V, Zeng Y, Lee TD, Ou X, Chen L, Lu SC. Induction of human methionine adenosyltransferase $2 \mathrm{~A}$ expression by tumor necrosis factor alpha. Role of NF-kappa B and AP-1. J Biol Chem 278: 50887-50896, 2003.

410. Yang H, Zheng Y, Li TW, Peng H, Fernandez-Ramos D, Martinez-Chantar ML, Rojas AL, Mato JM, Lu SC. Methionine adenosyltransferase 2B, HuR, and sirtuin 1 protein cross-talk impacts on the effect of resveratrol on apoptosis and growth in liver cancer cells. J Biol Chem 288: 23161-23670, 2013.

411. Yang HB, Xu YY, Zhao XN, Zou SW, Zhang Y, Zhang M, Li JT, Ren F, Wang LY, Lei QY. Acetylation of MAT IIalpha represses tumour cell growth and is decreased in human hepatocellular cancer. Nat Commun 6: 6973, 2015.

412. Yeo EJ, Briggs WT, Wagner C. Inhibition of glycine N-methyltransferase by 5methyltetrahydrofolate pentaglutamate. J Biol Chem 274: 37559-37564, 1999. 
Pajares and Pérez-Sala

413. Yie Y, Zhao S, Tang Q, Zheng F, Wu J, Yang L, Deng S, Hann SS. Ursolic acid inhibited growth of hepatocellular carcinoma HepG2 cells through AMPKalphamediated reduction of DNA methyltransferase 1. Mol Cell Biochem 402: 63-74, 2015.

414. Yin P, Zhao C, Li Z, Mei C, Yao W, Liu Y, Li N, Qi J, Wang L, Shi Y, Qiu S, Fan J, Zha X. Sp1 is involved in regulation of cystathionine gamma-lyase gene expression and biological function by PI3K/Akt pathway in human hepatocellular carcinoma cell lines. Cell Signal 24: 1229-1240, 2012.

415. Yuan G, Vasavda C, Peng YJ, Makarenko VV, Raghuraman G, Nanduri J, Gadalla MM, Semenza GL, Kumar GK, Snyder SH, Prabhakar NR. Protein kinase Gregulated production of H2S governs oxygen sensing. Sci Signal 8: ra37, 2015.

416. Zeisel SH, Wurtman RJ. Dietary intake of methionine: Influence of brain Sadenosylmethionine. In: Transmethylation. edited by Usdin E, Borchardt RT, Creveling CR. New York: Elsevier; 1979. pp. 59-68.

417. Zhang JJ, Chen JT, Hua L, Yao KH, Wang CY. miR-98 inhibits hepatocellular carcinoma cell proliferation via targeting EZH2 and suppressing Wnt/beta-catenin signaling pathway. Biomed Pharmacother 85: 472-478, 2017.

418. Zhang T, Wei W, Dirsch O, Kruger T, Kan C, Xie C, Kniemeyer O, Fang H, Settmacher U, Dahmen U. Identification of Proteins Interacting with Cytoplasmic HighMobility Group Box 1 during the Hepatocellular Response to Ischemia Reperfusion Injury. Int J Mol Sci 18: E167, 2017.

419. Zhang W, Braun A, Bauman Z, Olteanu H, Madzelan P, Banerjee R. Expression profiling of homocysteine junction enzymes in the NCI60 panel of human cancer cell lines. Cancer Res 65: 1554-1560, 2005.

420. Zhang Y, Yang X, Gui B, Xie G, Zhang D, Shang Y, Liang J. Corepressor protein CDYL functions as a molecular bridge between polycomb repressor complex 2 
Pajares and Pérez-Sala

and repressive chromatin mark trimethylated histone lysine 27. J Biol Chem 286: 42414-42425, 2011.

421. Zhao Y, Wu S, Gao X, Zhang Z, Gong J, Zhan R, Wang X, Wang W, Qian L. Inhibition of cystathionine beta-synthase is associated with glucocorticoids oversecretion in psychological stress-induced hyperhomocystinemia rat liver. Cell Stress Chaperones 18: 631-641, 2013.

422. Zheng F, Liao YJ, Cai MY, Liu YH, Liu TH, Chen SP, Bian XW, Guan XY, Lin MC, Zeng YX, Kung HF, Xie D. The putative tumour suppressor microRNA-124 modulates hepatocellular carcinoma cell aggressiveness by repressing ROCK2 and EZH2. Gut 61: 278-289, 2012.

423. Zhou Q, Agoston AT, Atadja P, Nelson WG, Davidson NE. Inhibition of histone deacetylases promotes ubiquitin-dependent proteasomal degradation of DNA methyltransferase 1 in human breast cancer cells. Mol Cancer Res 6: 873-883, 2008.

424. Zhou Y, Harrison DE, Love-Myers K, Chen Y, Grider A, Wickwire K, Burgess JR, Stochelski MA, Pazdro R. Genetic analysis of tissue glutathione concentrations and redox balance. Free Radic Biol Med 71: 157-164, 2014.

425. Zhuang $\mathrm{C}$, Wang $\mathrm{P}$, Huang $\mathrm{D}, \mathrm{Xu} \mathrm{L}$, Wang $\mathrm{X}$, Wang $\mathrm{L}, \mathrm{Hu} \mathrm{L}$. A doublenegative feedback loop between EZH2 and miR-26a regulates tumor cell growth in hepatocellular carcinoma. Int J Oncol 48: 1195-1204, 2016.

426. Zou K, Ouyang Q, Li H, Zheng J. A global characterization of the translational and transcriptional programs induced by methionine restriction through ribosome profiling and RNA-seq. BMC Genomics 18: 189, 2017. 


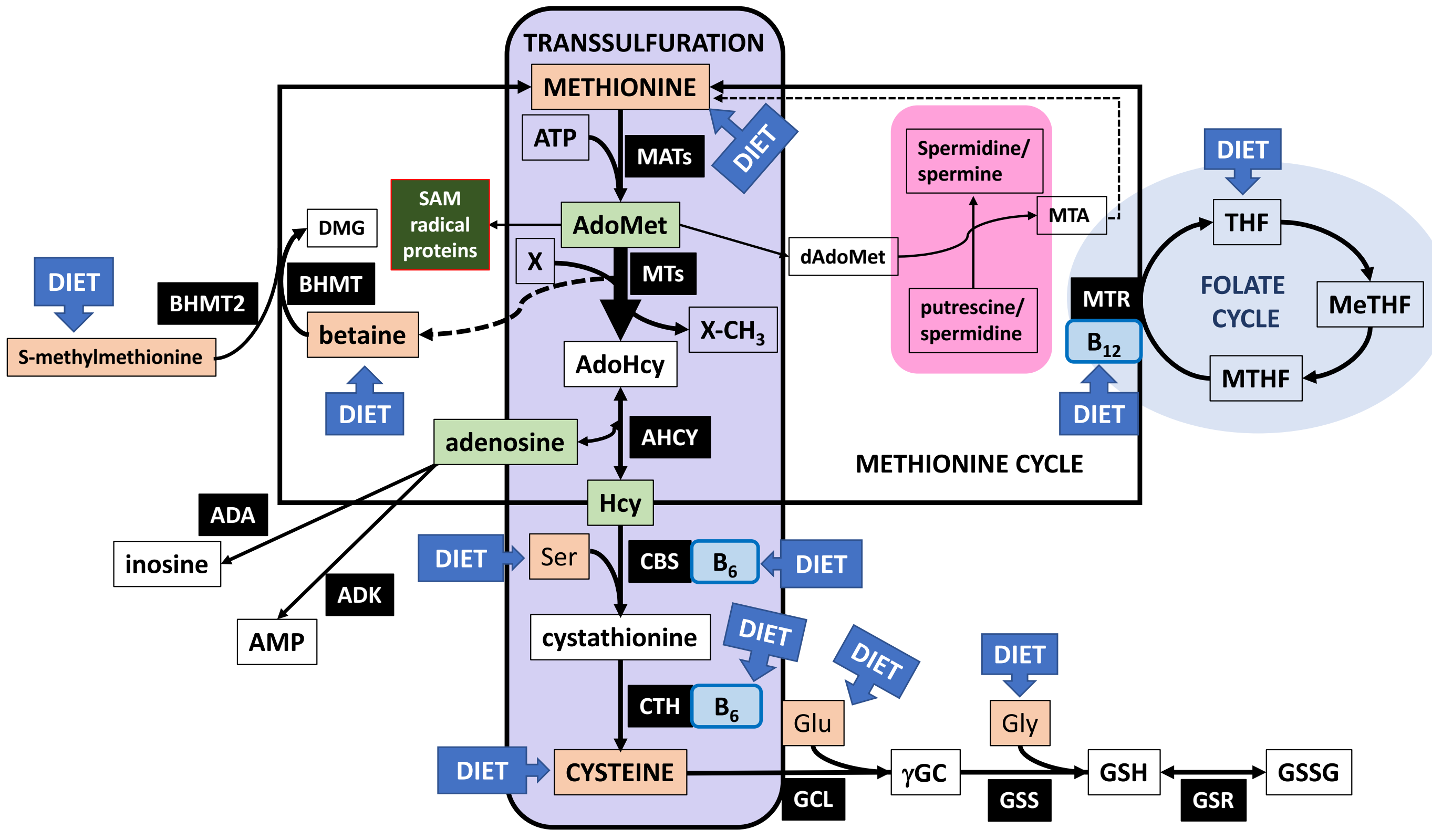


A
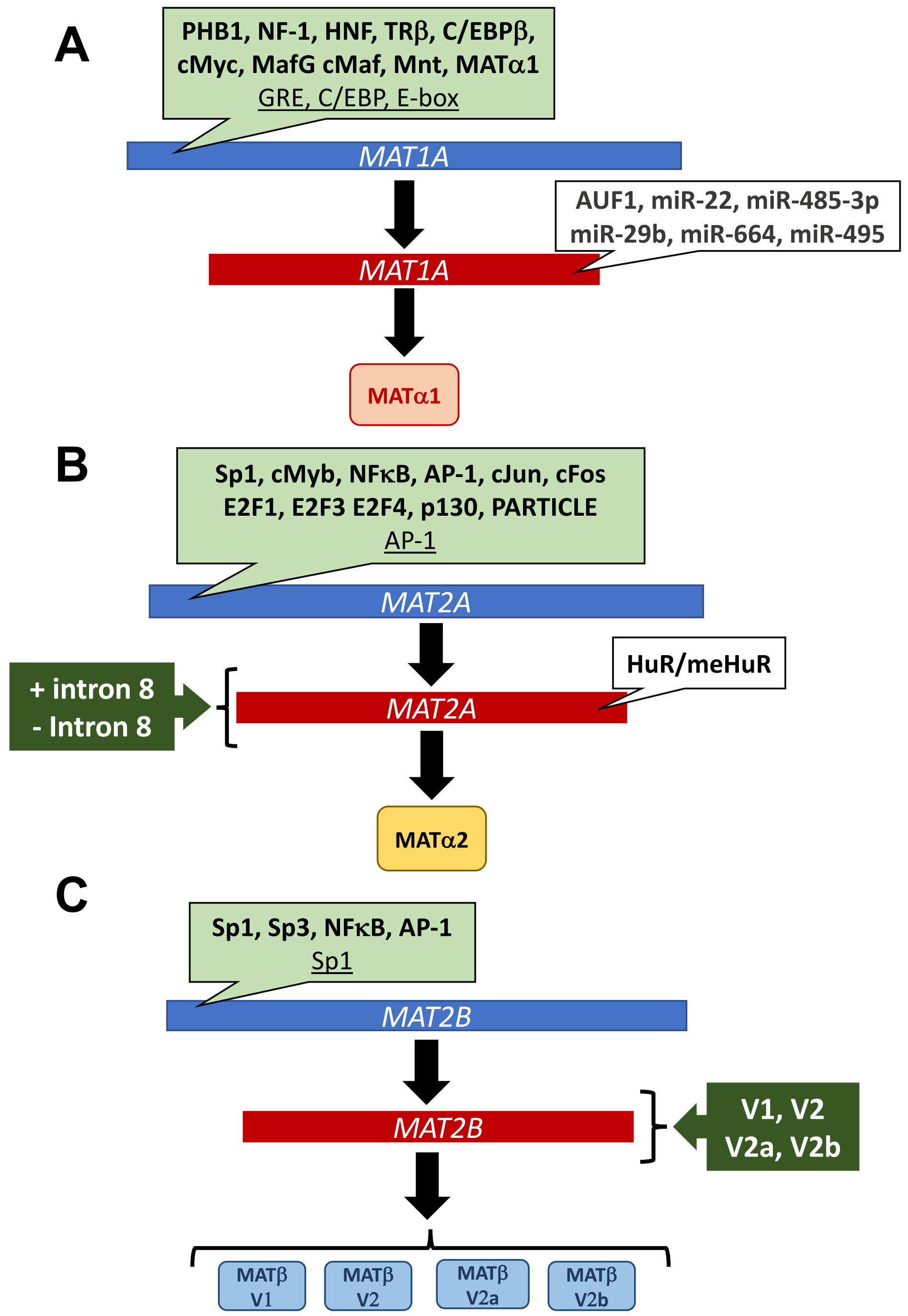


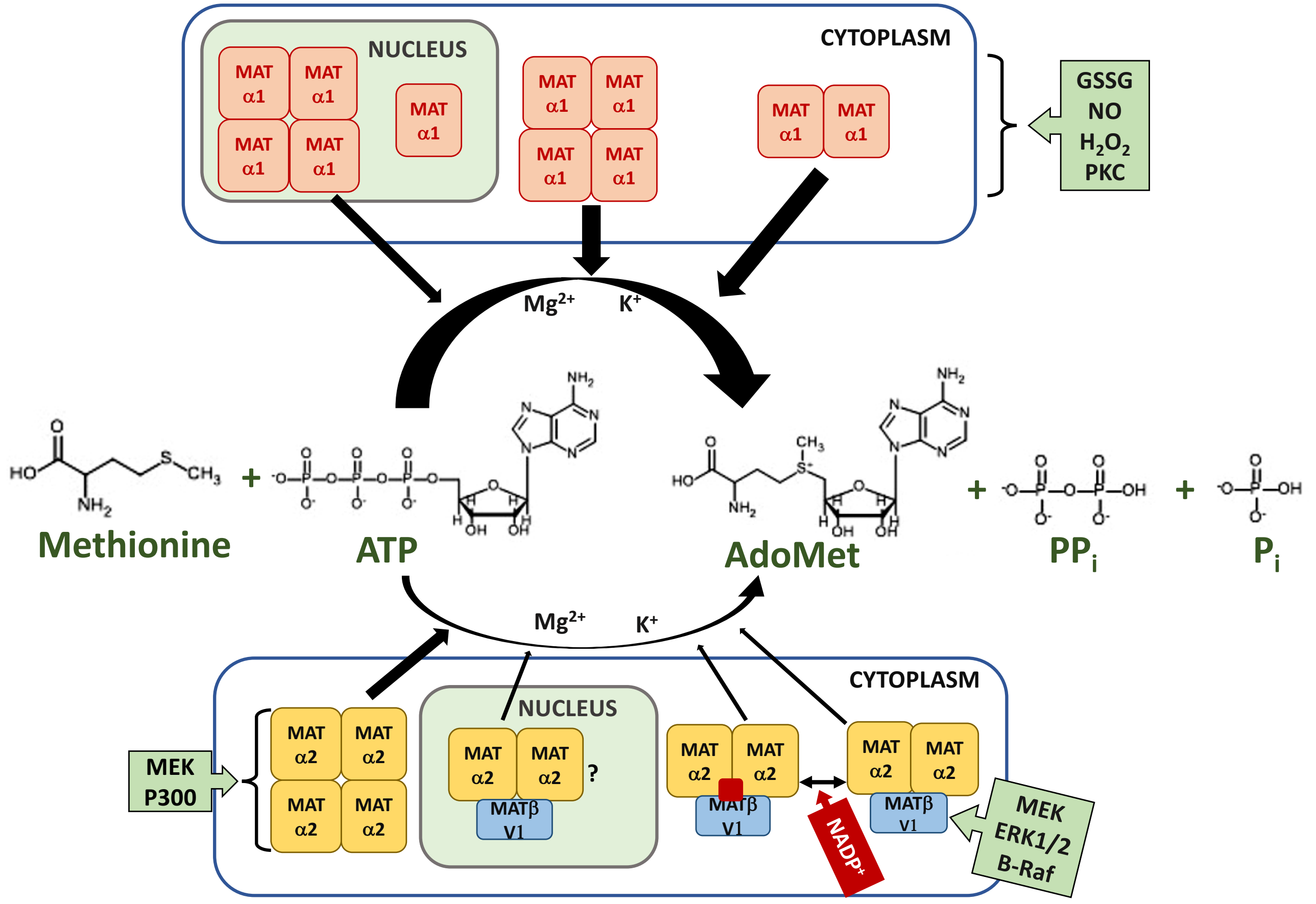


A
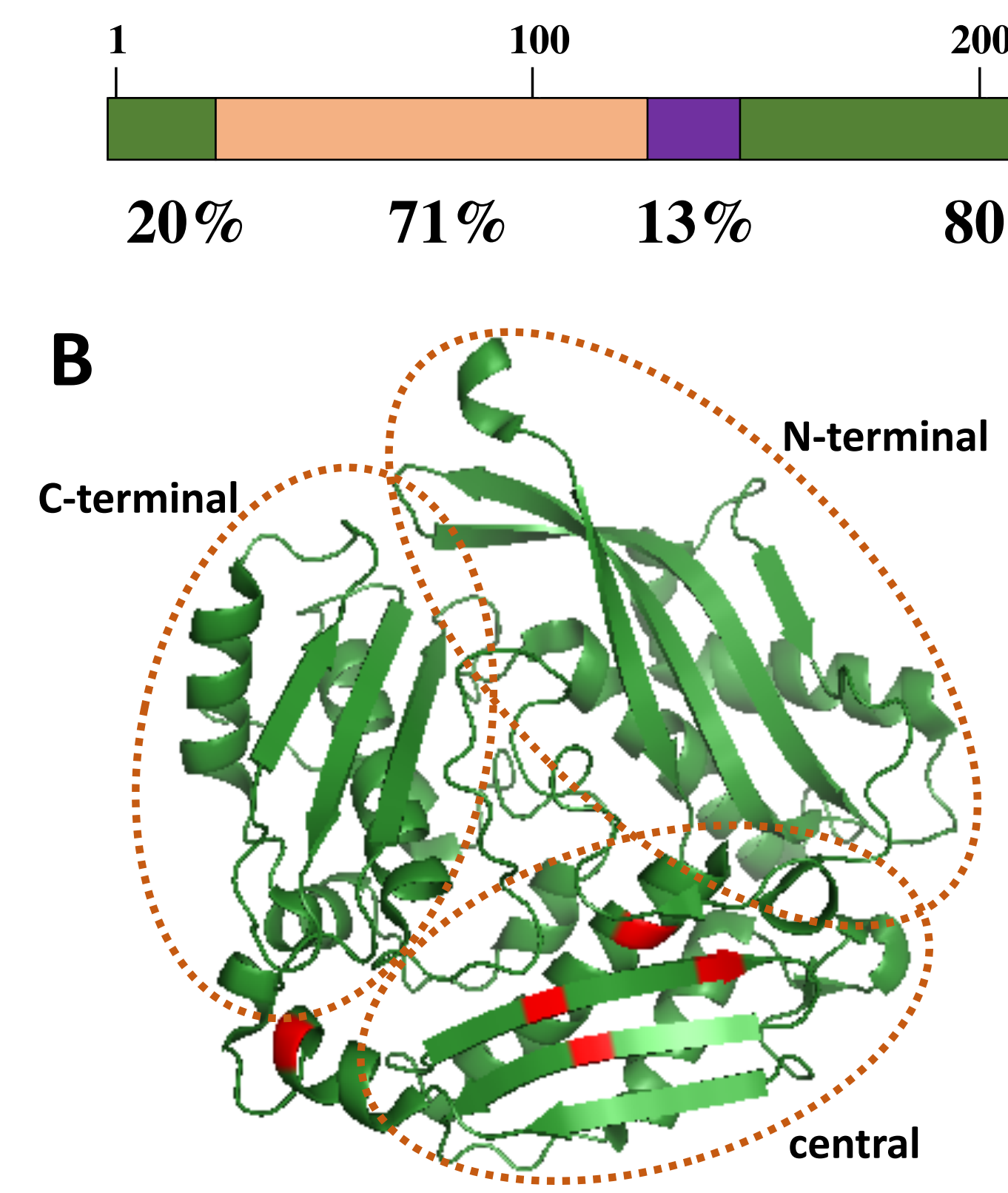

C

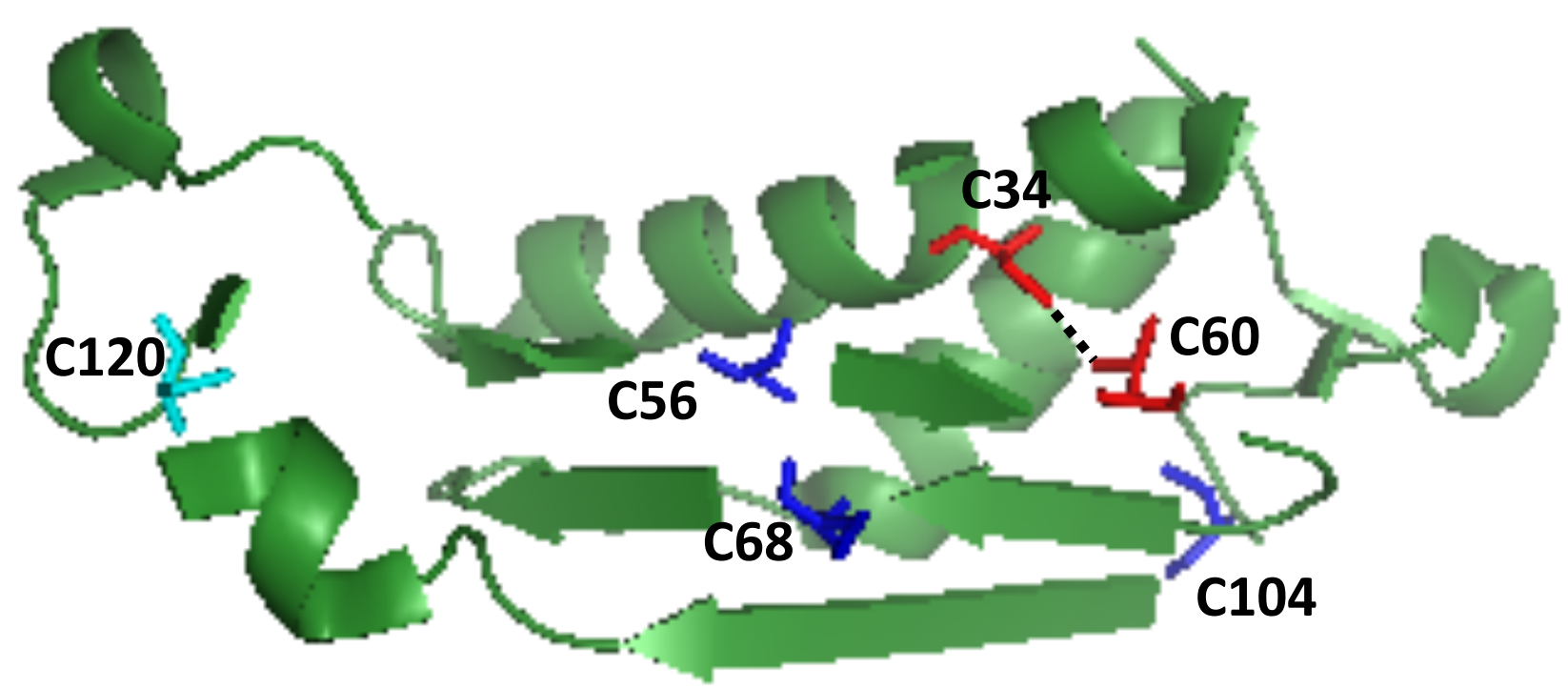



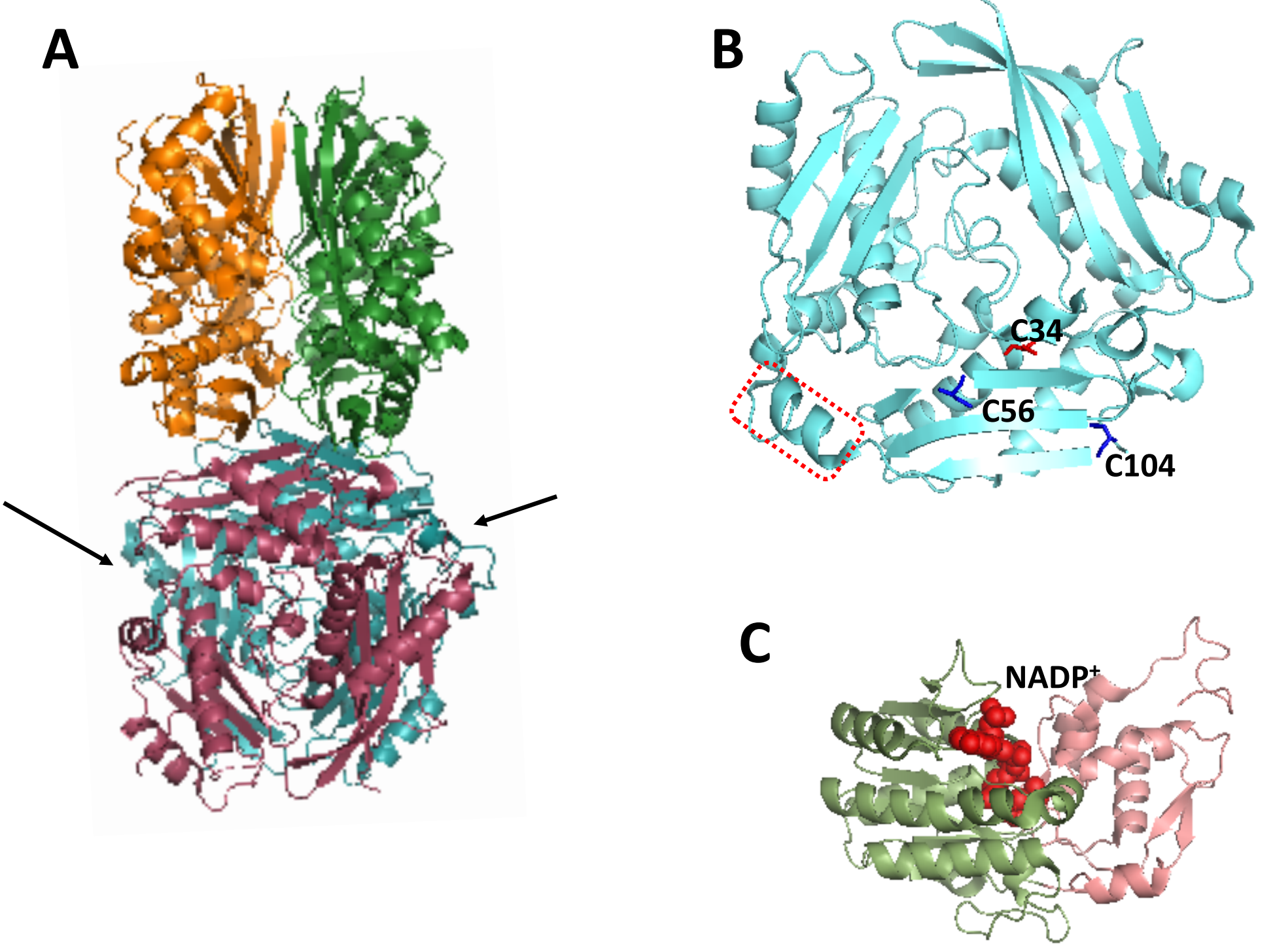


\section{CLASSICAL VIEW}

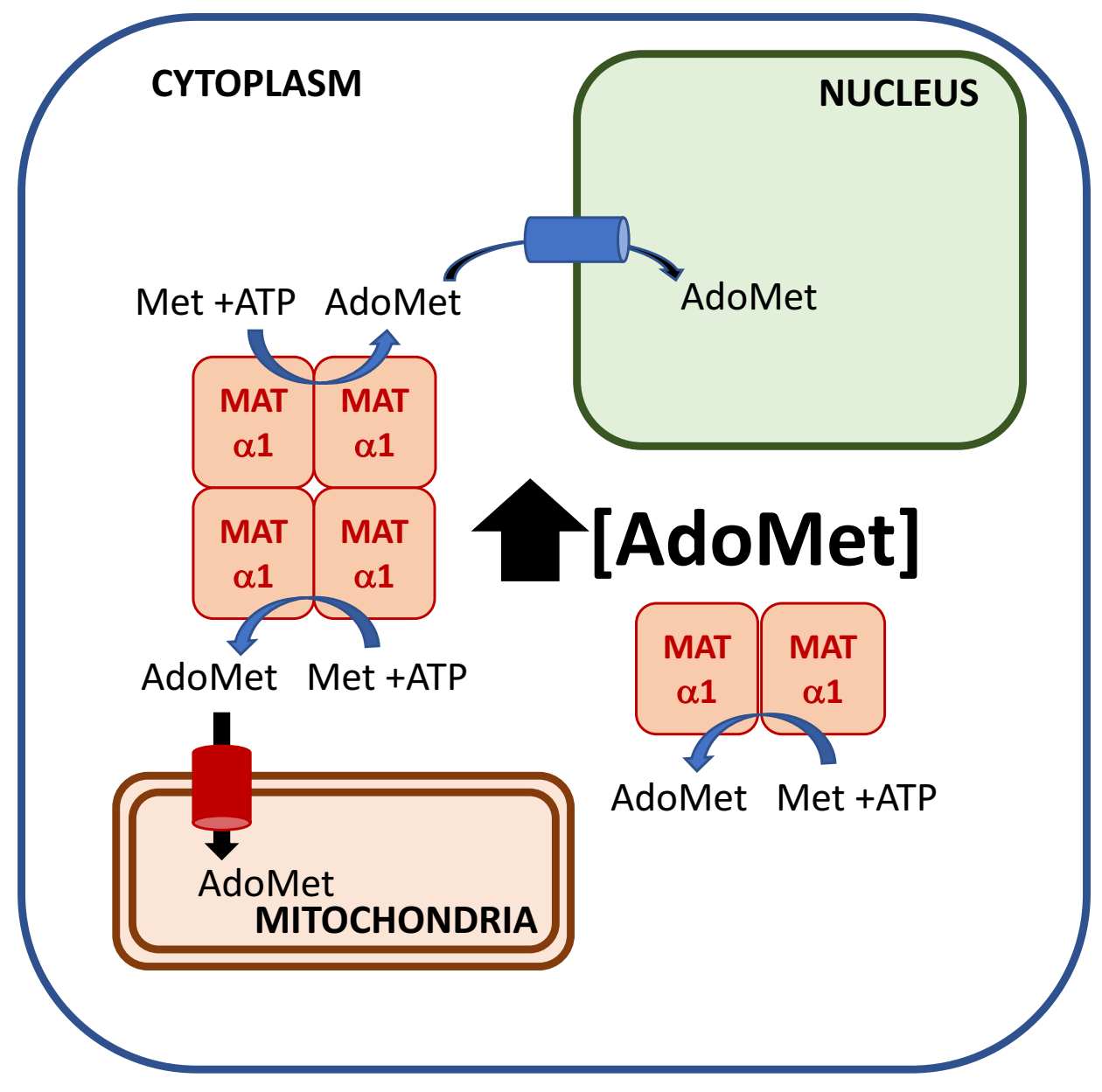

CURRENT VIEW

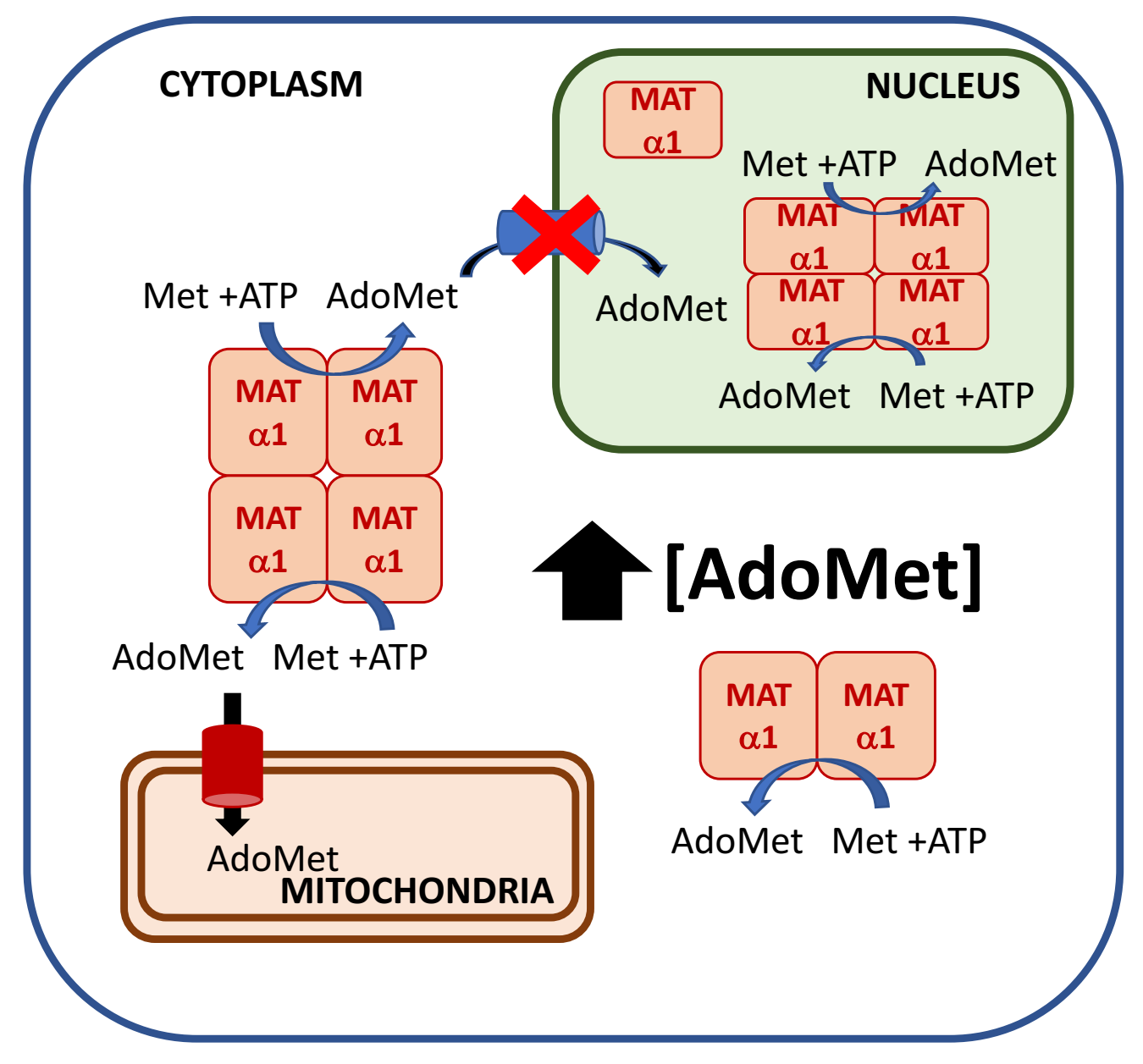




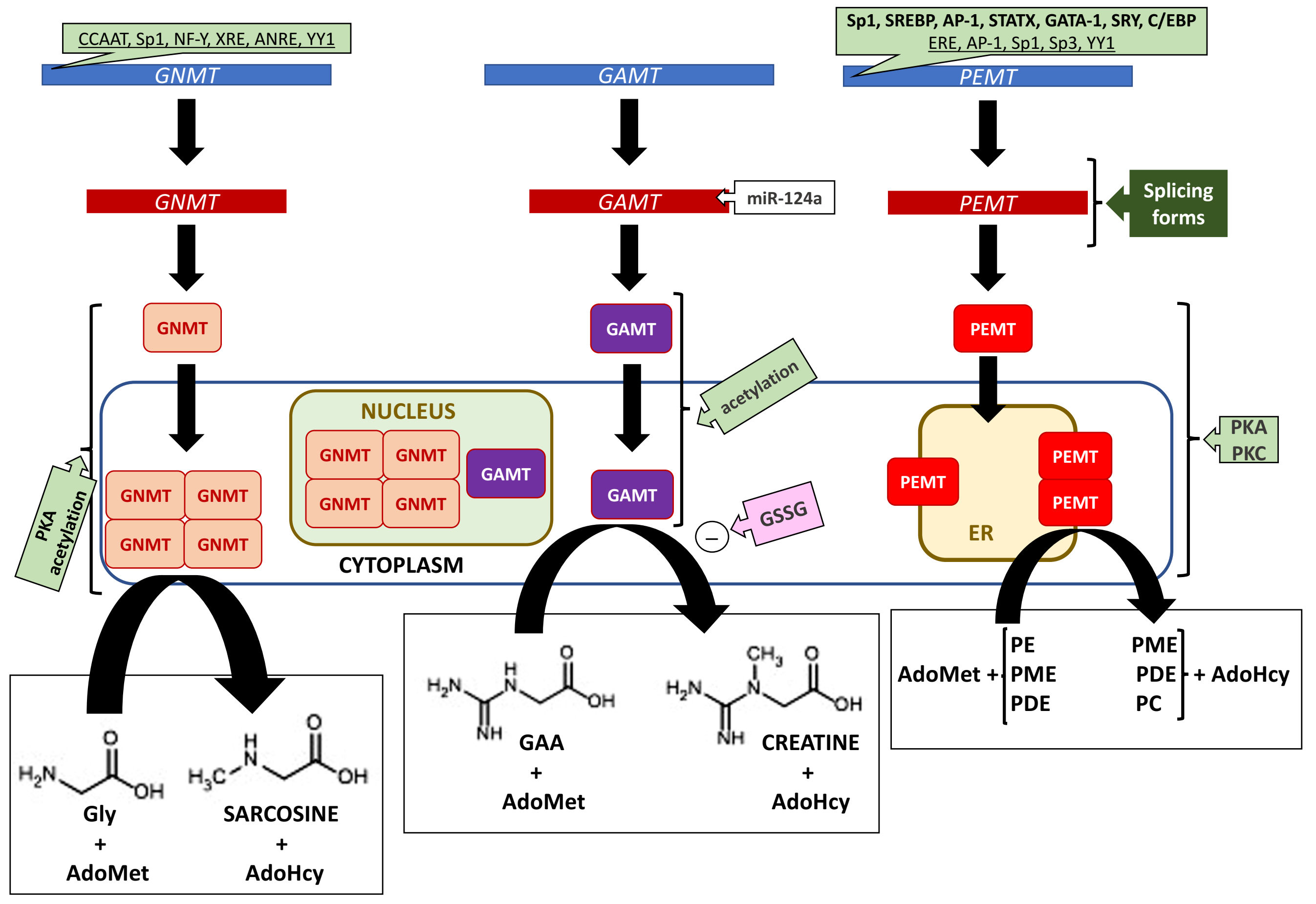


A

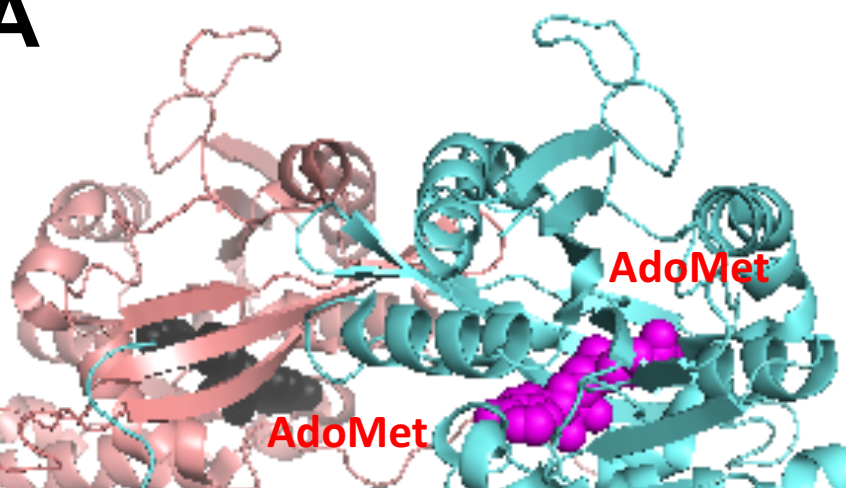

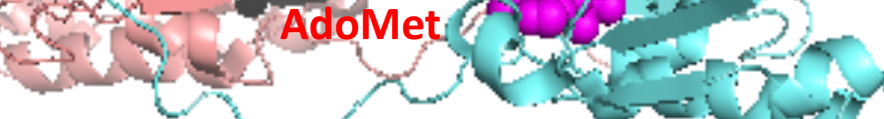

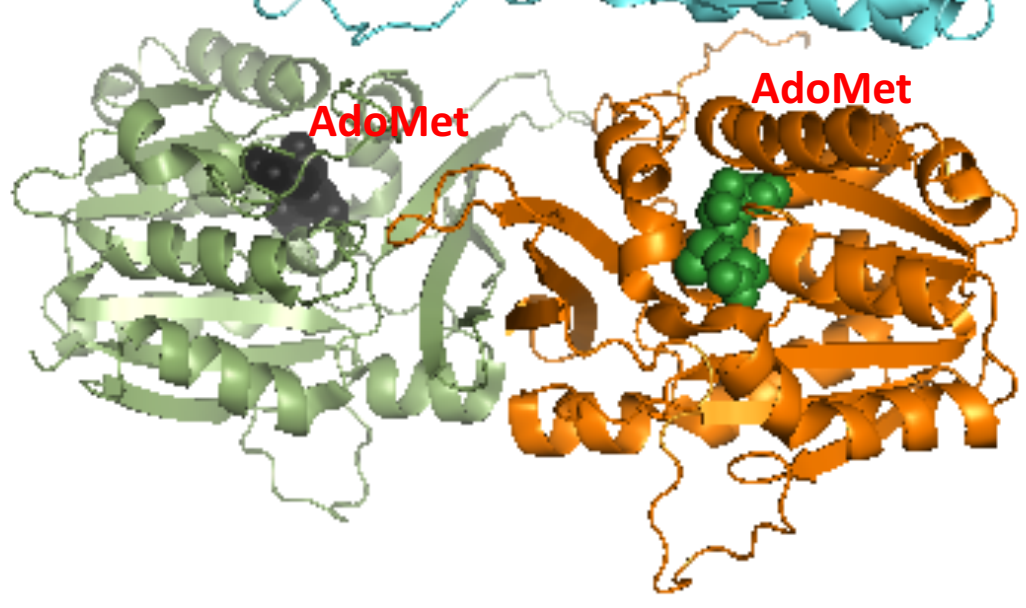

B

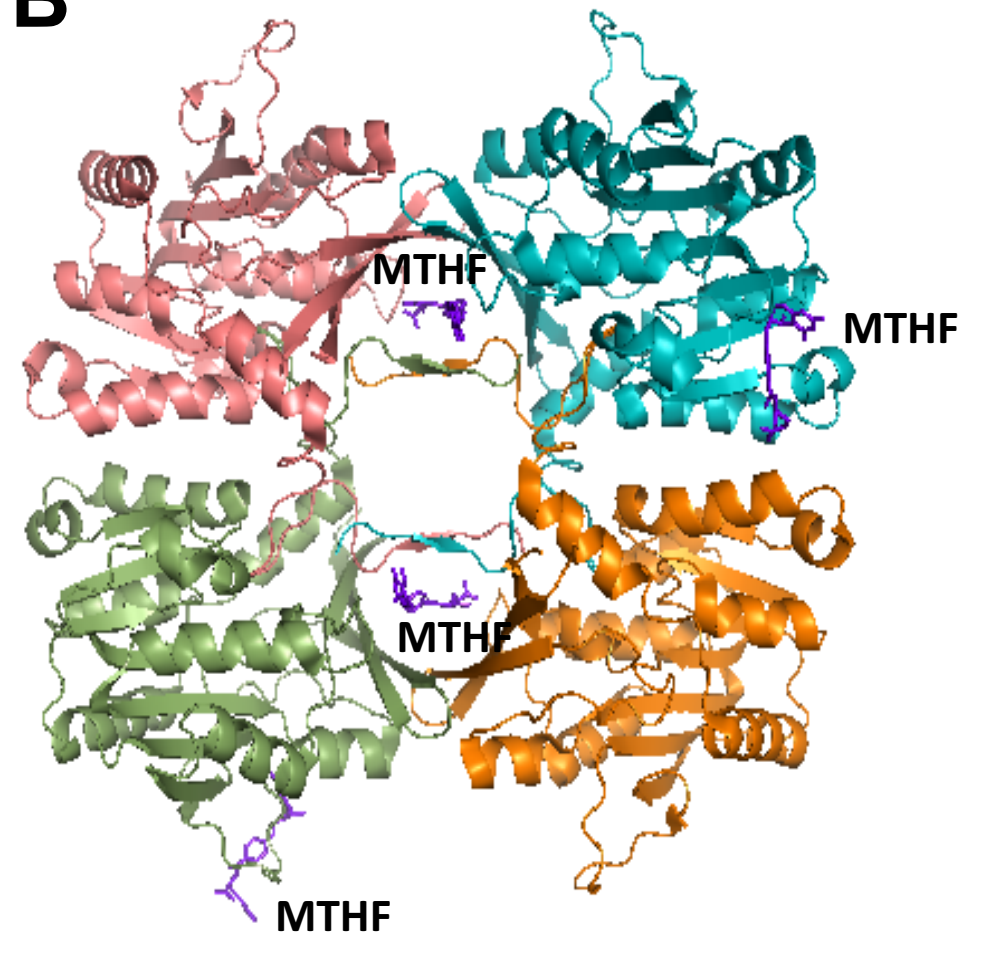

C

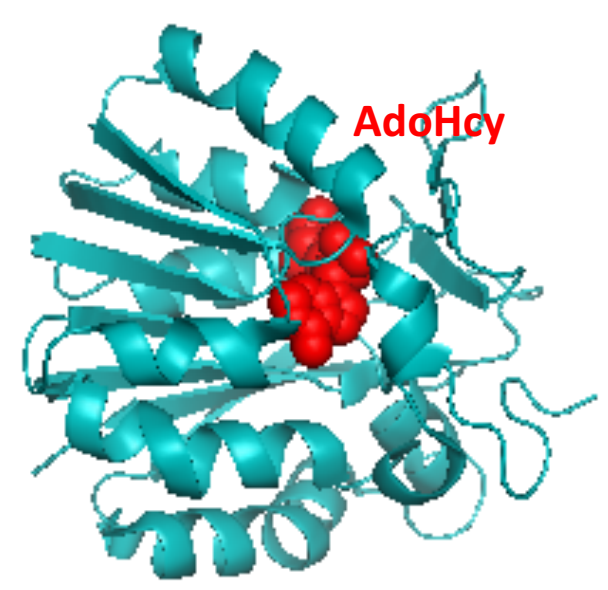




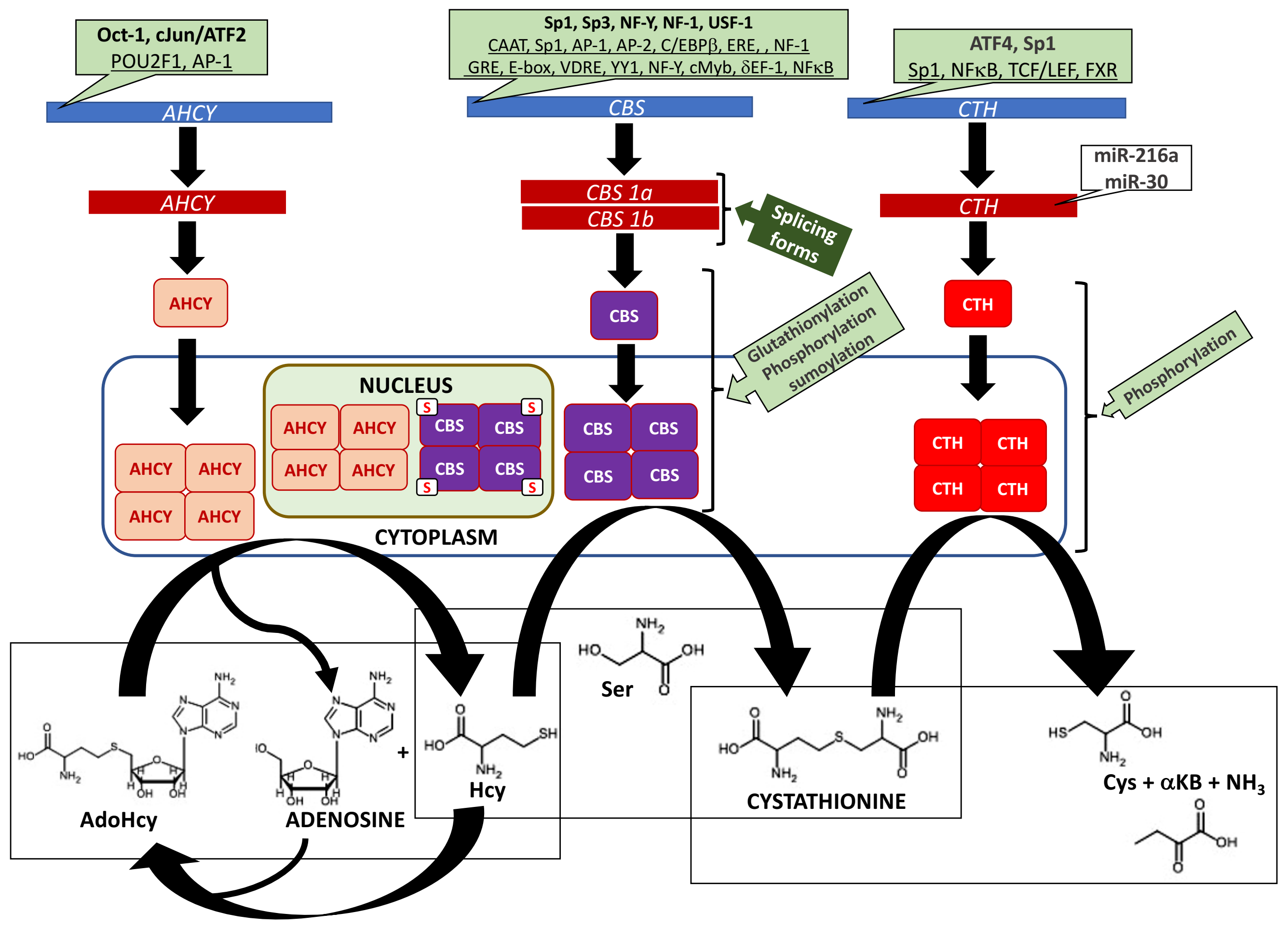


A

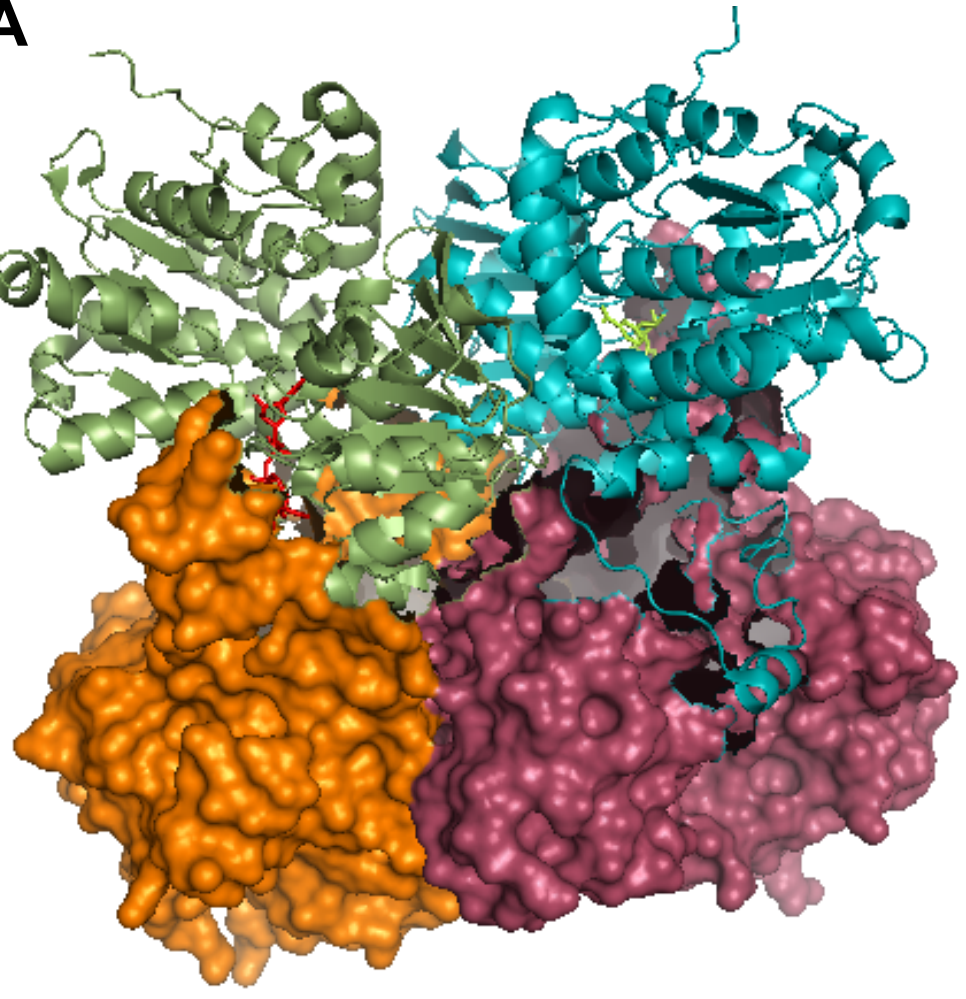

C

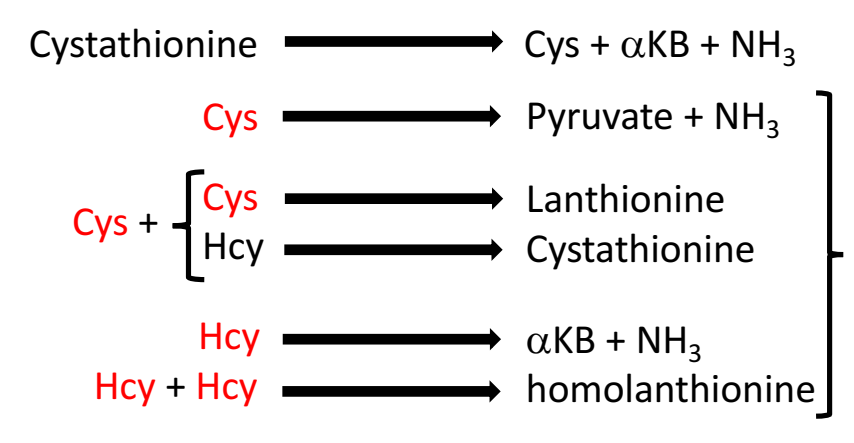

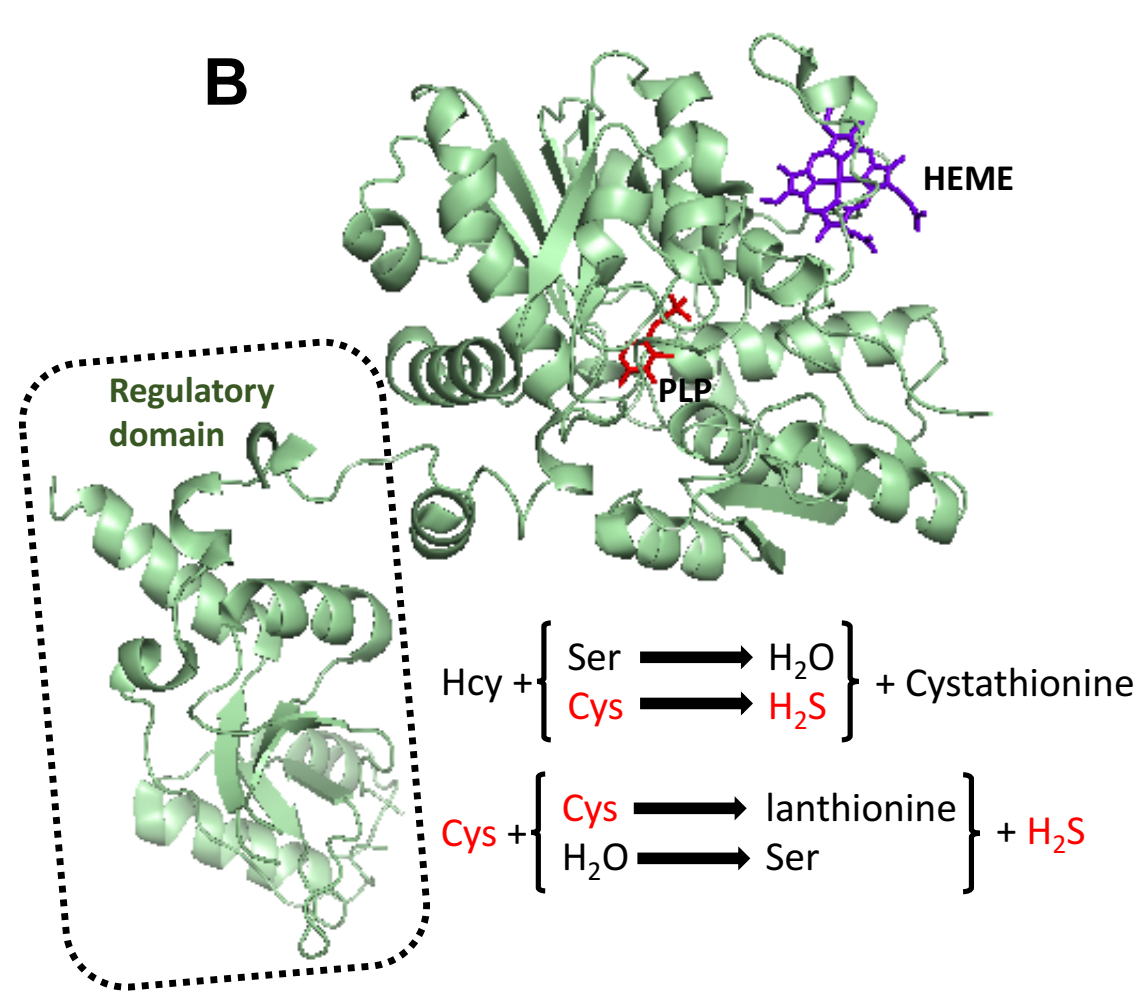

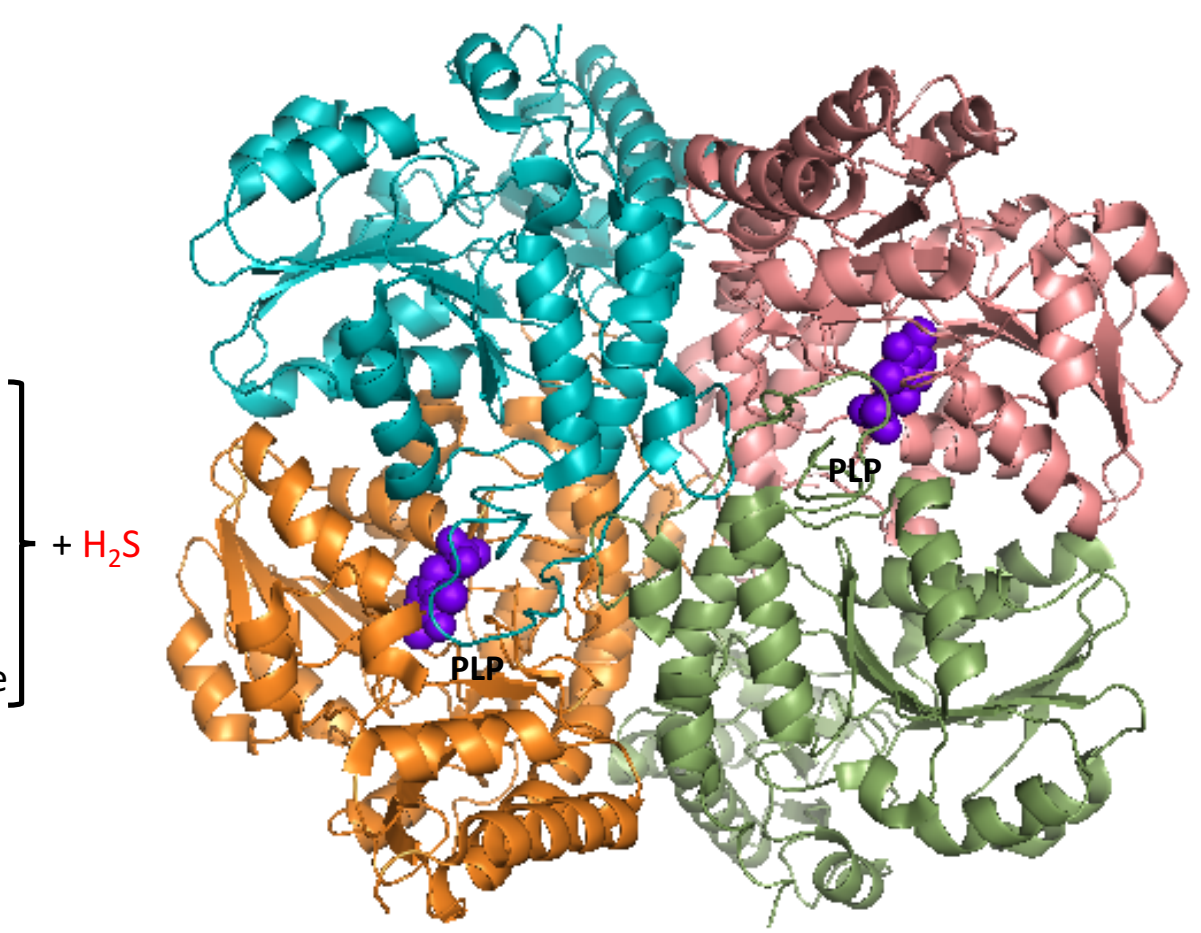




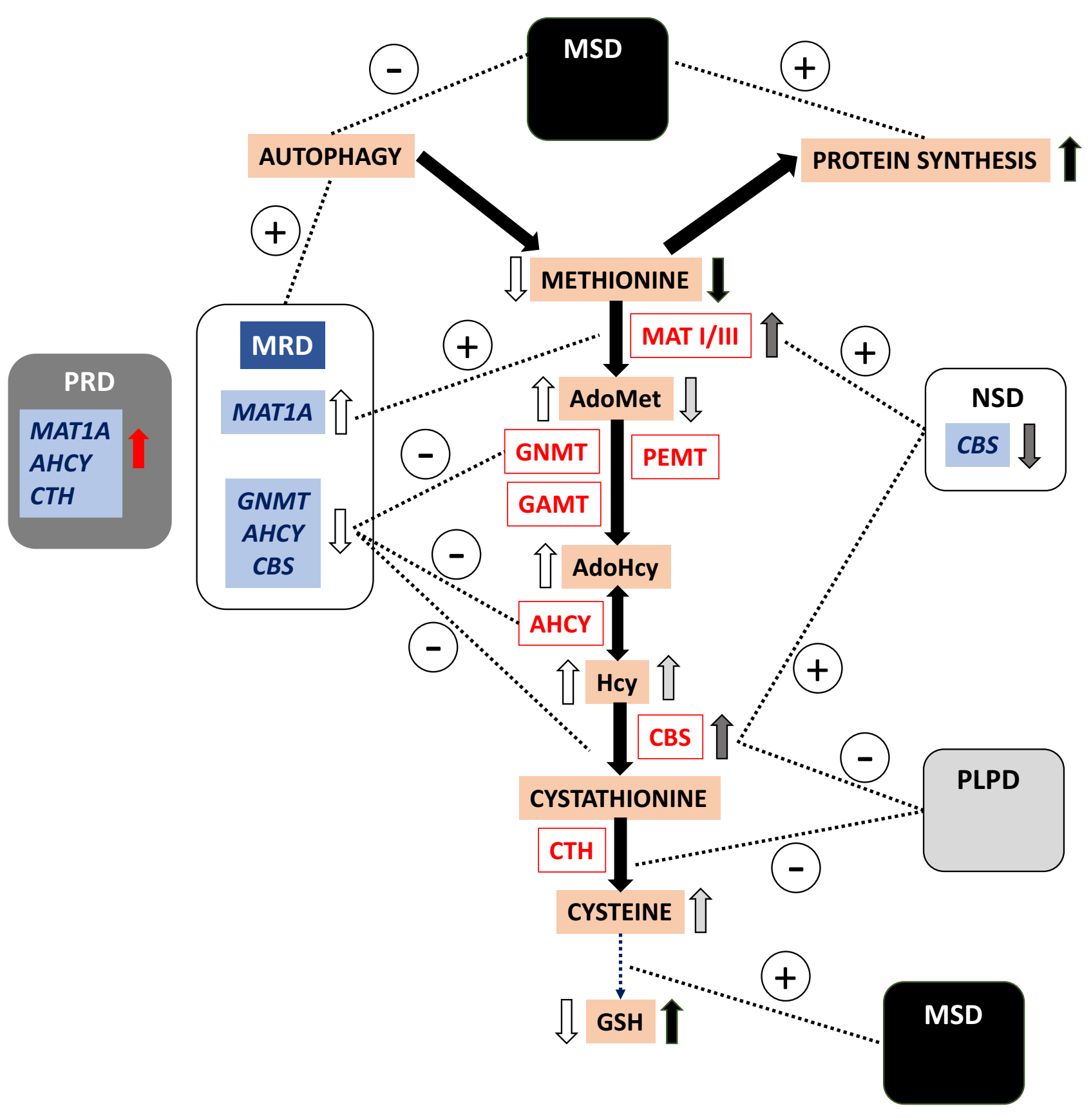




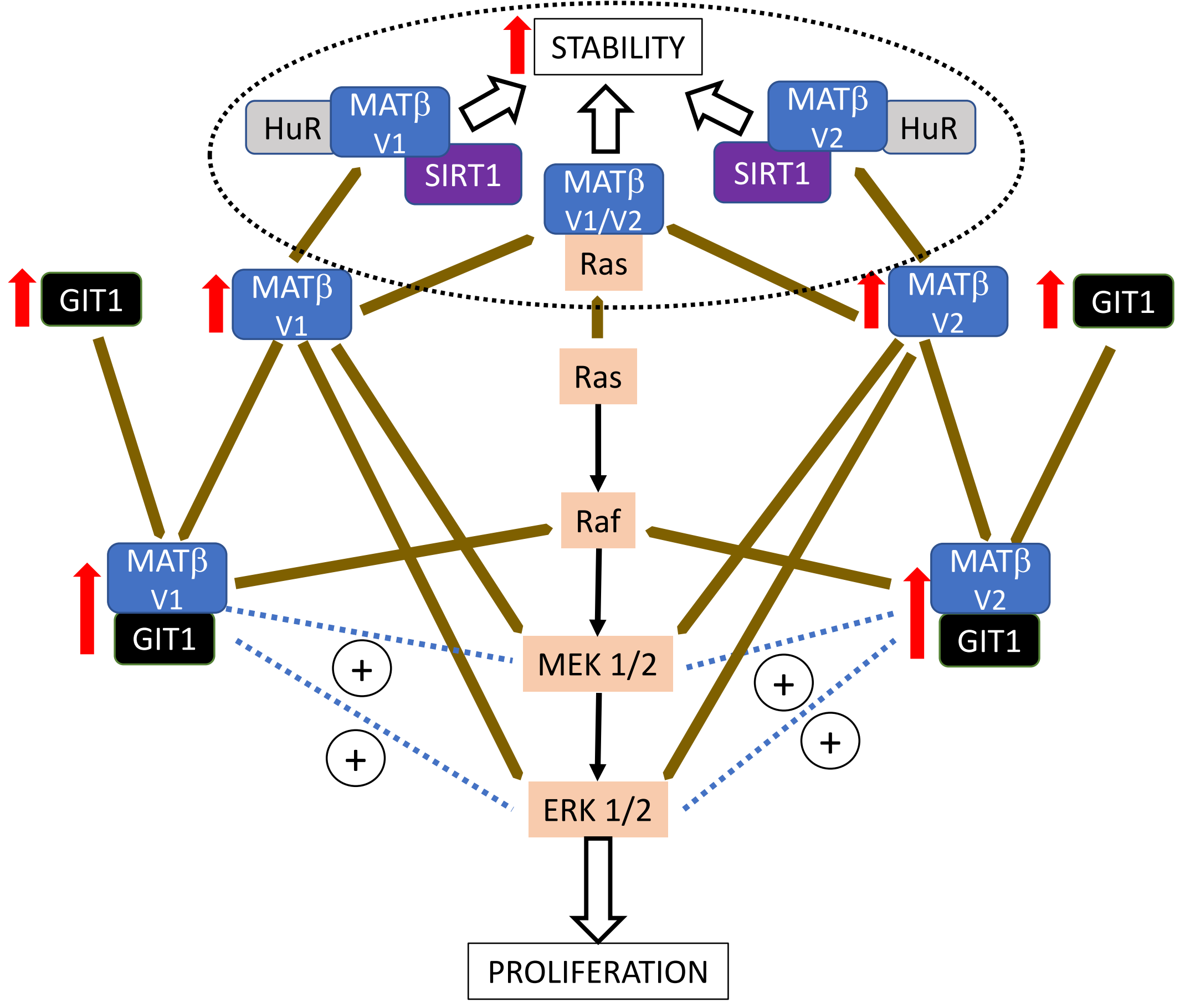



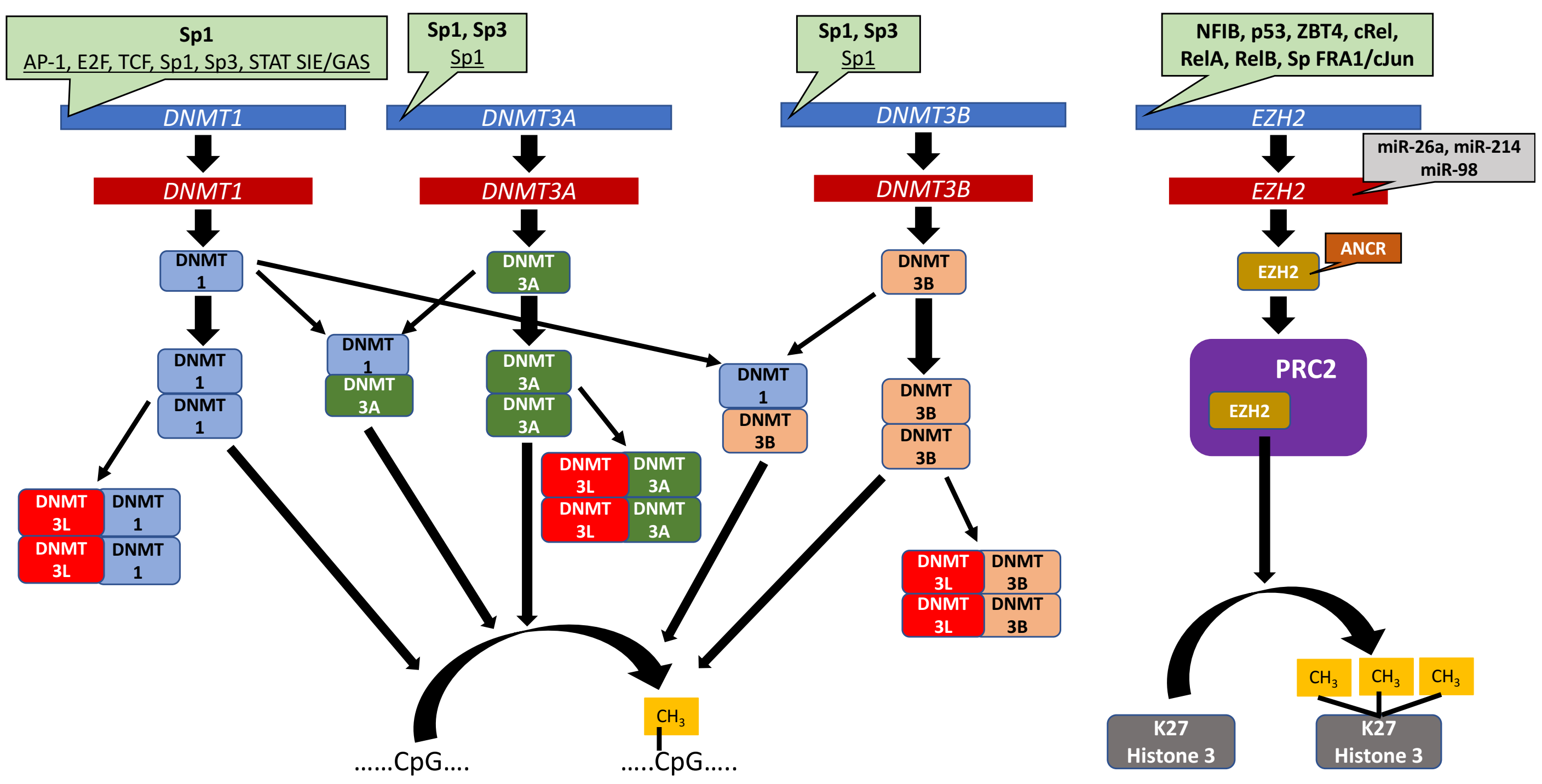


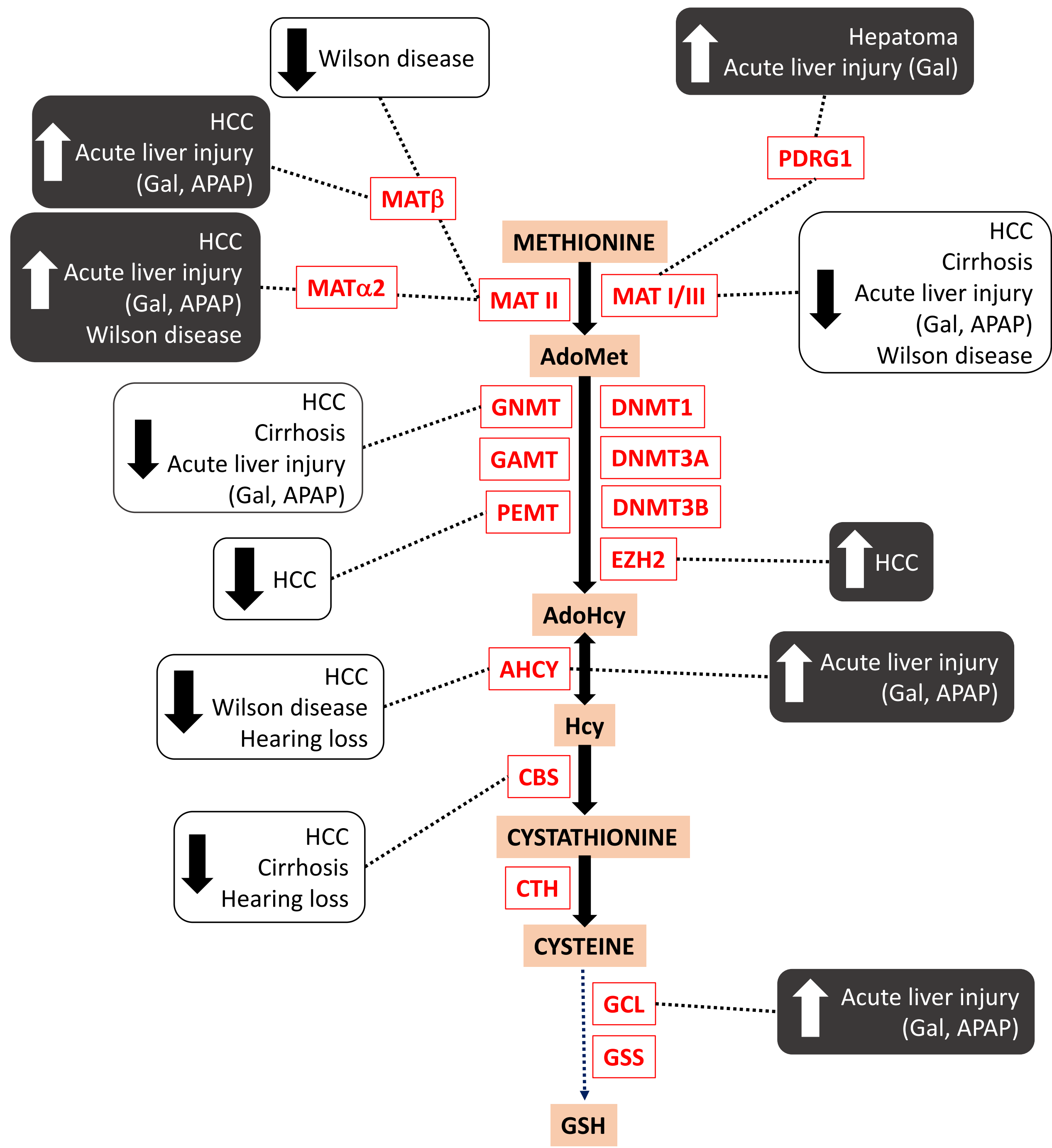




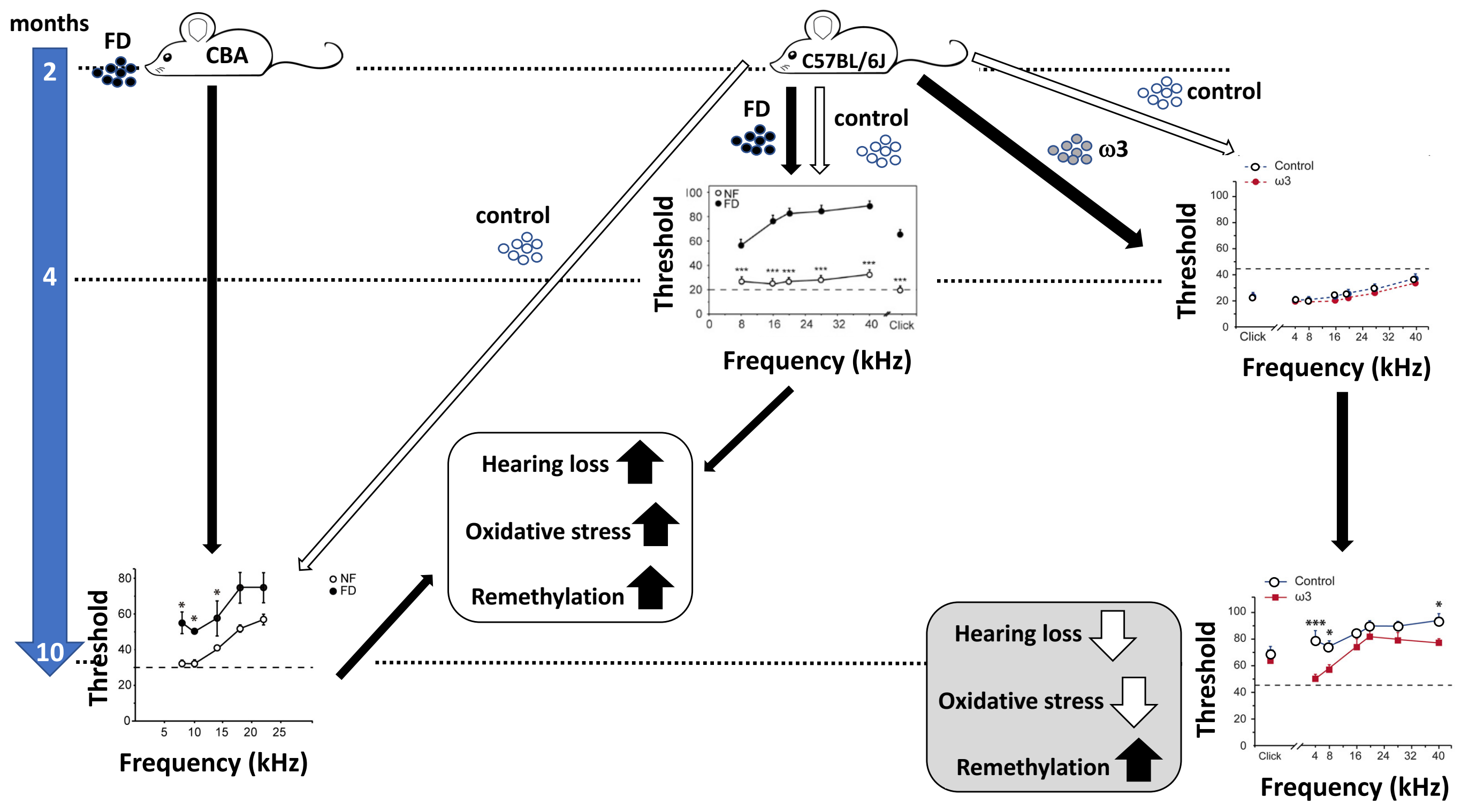




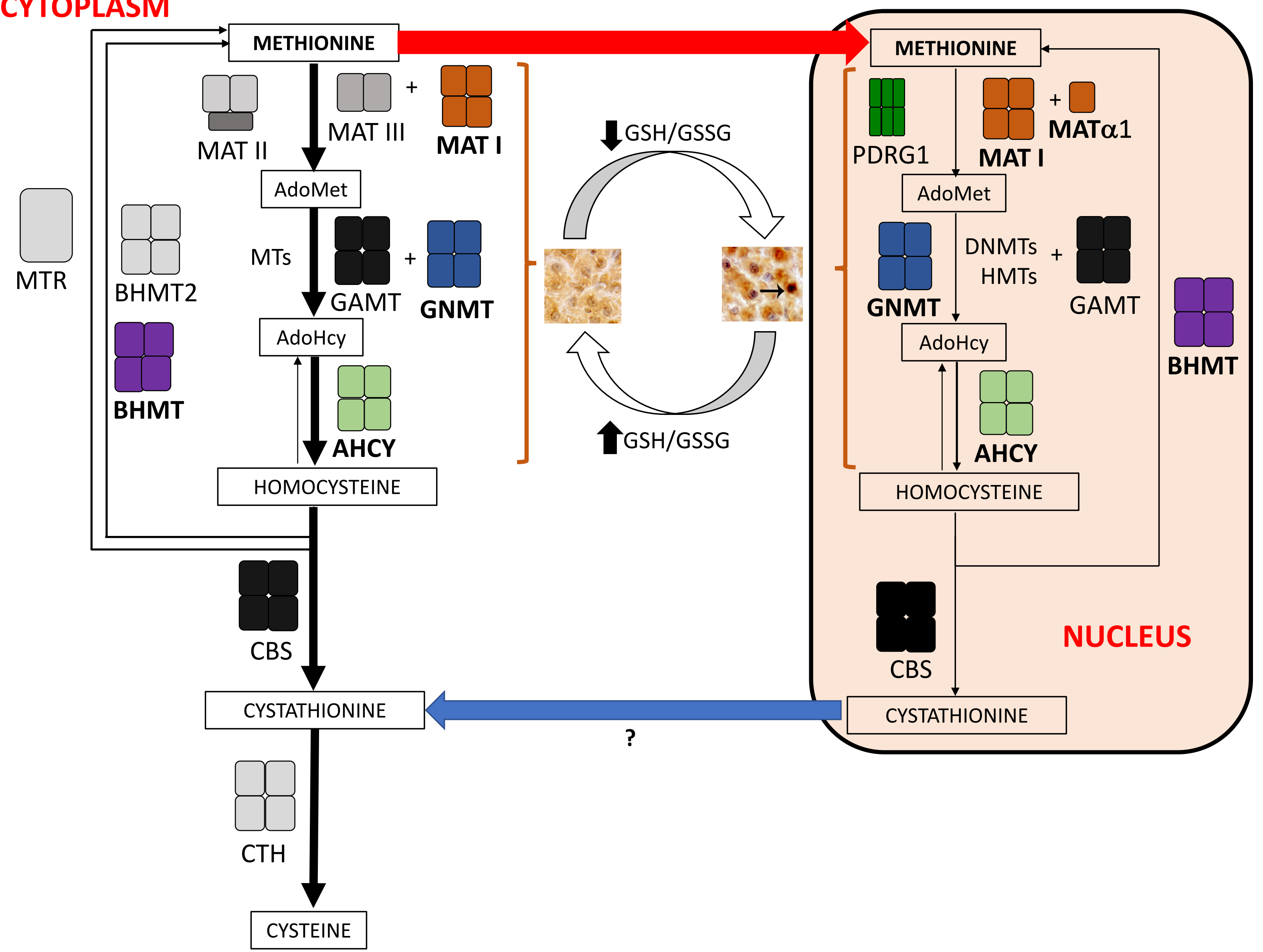




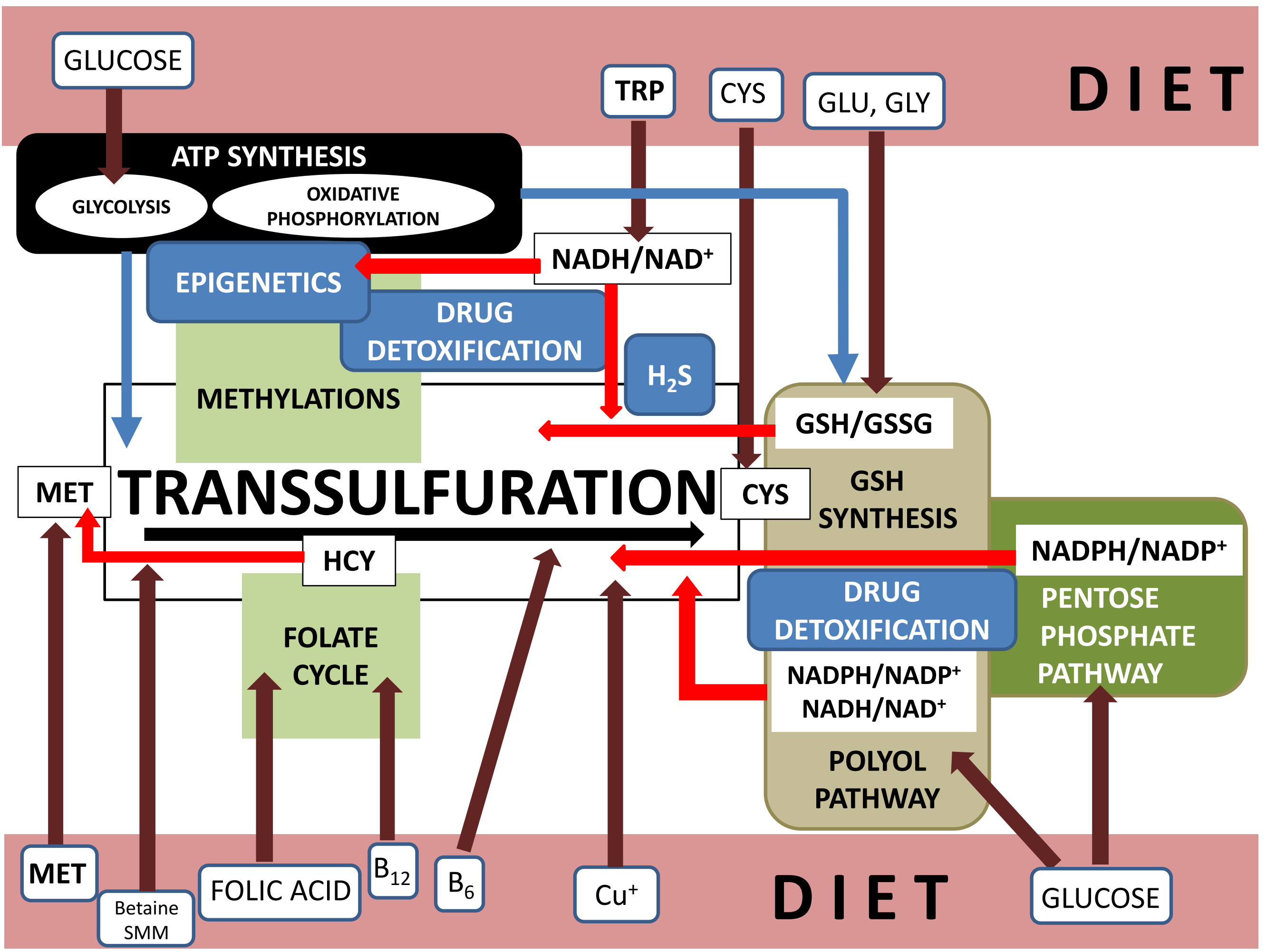

